\title{
Stereodivergent Synthesis of Biologically Active Spironucleoside Scaffolds via Catalytic Cyclopropanation of 4-exo-methylene Furanosides
}

Zeina Neouchy*a, Jonas Verhoeven ${ }^{b}$, Hanchu Kong ${ }^{c}$ Yongbin Zhaoc, Wenbin Wang ${ }^{c}$, Marta Brambilla $^{b}$, Kristof Van Hecke ${ }^{d}$, Lieven Meerpoel ${ }^{b}$, Jan Willem Thuring ${ }^{b}$, Guido Verniest ${ }^{b}$ and Johan Winne ${ }^{a}$

a Department of Organic and Macromolecular Chemistry. Ghent University, Krijgslaan 281 - S4, 9000 Ghent, Belgium

b Janssen Pharmaceutica NV, Turnhoutseweg 30, 2340 Beerse, Belgium

' Department of Synthetic Chemistry, Pharmaron Beijing Co., Ltd., 6 Taihe Road BDA, Beijing, 100176, China

${ }^{d}$ XStruct, Department of Chemistry. Ghent University, Krijgslaan 281 - S3, 9000 Ghent, Bel gium

\section{Supporting Information}

\section{Table of contents:}

General Information...............................................................................S-2

1. Ring contraction of 4-spirochlorocyclobutanone A....................................S-3

2. Optimization tables of the stereoselective cyclopropanation.......................S-5

3. Biological studies.................................................................................

4. Copies of NM R spectra...........................................................................S-8

5. Stereochemistry determination by 2D NM R spectroscopy ........................5-52

6. X-ray crystal structure of compound 10..................................................5-58 


\section{General information}

All reactions have been carried out in previously dried glass ware, using a magnetically stirred Teflon bar under argon atmosphere. Anhydrous and HPLC grade solvents were used as supplied, except $\mathrm{CH}_{2} \mathrm{Cl}_{2}$ which was distilled over $\mathrm{CaH}_{2}$ prior to use. Most of the reagents were purchased from commercial sources except methyl 2,3-isopropylidene-4-methylidene-D-erythro furanoside $\mathbf{1}$, 1,2-isopropylidene-3-hydroxy-4-methylidene-L-threo furanose and its D-erythro epimer which were synthesized according to literature procedure ${ }^{1}$ as well as (R)-Ph-Pheox-Ru(II) catalyst which was prepared according to a literature procedure. ${ }^{2}$ Synthesized products and intermediates were stored under argon in the dark at $-20^{\circ} \mathrm{C}$. Thin layer chromatography (TLC) was performed on M erck $60 \mathrm{~F}_{254}$ silica coated aluminum-backed plates (thickness of from 175 to $225 \mu \mathrm{m}$ ) using petroleum ether (PE)/EtOAc as eluent and visualized with UV lamp $(254 \mathrm{~nm})$, and by treatment with a solution of p-anisaldehyde/ $\mathrm{H}_{2} \mathrm{SO}_{4} / \mathrm{AcOH}$ in ethanol followed by heating. Flash chromatography was carried out with ROCC silica (particle size 60-200 $\mu \mathrm{m}$ ). The NMR spectra were recorded on a Bruker 300, 400 or $500 \mathrm{M} \mathrm{Hz}$. The chemical shifts $\delta$ are reported in ppm relative to chloroform-d $\left(\mathrm{CDCl}_{3}: \delta_{\mathrm{H}}=7.26 \mathrm{ppm}\right.$ and $\left.\delta_{\mathrm{C}}=77.0 \mathrm{ppm}\right)$, dichloromethane- $\mathrm{d}_{2}\left(\mathrm{CD}_{2} \mathrm{Cl}_{2}: \delta_{\mathrm{H}}=5.32 \mathrm{ppm}\right.$ and $\left.\delta_{\mathrm{C}}=54.0 \mathrm{ppm}\right)$, DM SO $-\mathrm{d}_{6}\left(\delta_{\mathrm{H}}=\right.$ $2.50 \mathrm{ppm}$ and $\delta_{\mathrm{C}}=39.5 \mathrm{ppm}$ ) or methanol- $\mathrm{d}_{4}\left(\delta_{\mathrm{H}}=3.31 \mathrm{ppm}\right.$ and $\left.\delta_{\mathrm{C}}=49.0 \mathrm{ppm}\right)$. The multiplicity is designated by the following abbreviations: $s=$ singlet, $d=$ doublet, $t=$ triplet, $d d=$ doublet of doublets, $\mathrm{dt}=$ doublet of triplets, $\mathrm{ddd}=$ doublet of doublets of doublets, $q \mathrm{~d}=$ quartet of doublets, $\mathrm{m}$ $=$ multiplet, br =broad. Coupling constants $\mathrm{J}$ are reported in Hertz (Hz).The GCM S measurement was performed using an Agilent Gas Chromatography system (GC 6890) coupled to a Mass Spectrometer (MSD 5973) in which the MS detector was configured with an electronic impact ionization source (EI). High-resolution mass spectra (HRMS) were measured with an Agilent Accurate-Mass Quadrupole Time-of-Flight mass spectrometer using Electro Spray Ionization (ESI). SFC purifications were performed on a Acquity UPC2 system from Waters ${ }^{T M}$ equipped with a UV, QDA and ELSD detector. The SFC measurement was performed using an Analytical Supercritical fluid chromatography (SFC) system composed by a binary pump for delivering carbon dioxide $\left(\mathrm{CO}_{2}\right)$ and modifier, an autosampler, a column oven, a diode array detector equipped with a high-pressure flow cell standing up to 400 bars. Infrared (IR) spectra were obtained with a Perkin-Elmer 1000 FT-IR spectrometer equipped with a HATR module. Optical rotations were measured with a Perkin-Elmer 241 Polarimeter in a $10 \mathrm{~cm}$ cell. The concentrations indicated are in $\mathrm{g} / 100 \mathrm{~mL}$. The prefixes and designation of the cyclopropanation products are identified as follows: $\alpha$ for the top-faced cyclopropanation; $\beta^{\prime}$ for the bottom-faced cyclopropanation; trans/cis for the relation between the configuration of the ester group and the endocyclic $\mathrm{C}-\mathrm{O}$ bond of the furanose backbone.

\footnotetext{
${ }^{1}$ a) Verhoeven, J.; De Vleeschouwer, F.; Kong, H.; Van Hecke, K.; Pande, V.; Sun, W.; Vos, A.; Wu, T.; Meerpoel, L.; Thuring, J-W.; Verniest, G. Chem. Eur. J. 2019, 25, 15419-15423; b) Verhoeven, J.; Brambilla, M.; Chinta, N.; Hullaert, J. J. A.; Jouffroy, L. M.; Meerpoel, L.; Neouchy, Z; Thuring, J.W. J. F.; Verniest, G. A. F.; Winne, J. M. 2020, WO/2020/249663.

${ }^{2}$ Chanthamath, S.; Phomkeona, K.; Shibatomi, K.; Iwasa, S. Chem. Commun. 2012, 48, 7750-7752.
} 


\section{Ring contraction of 4-spirochlorocyclobutanone A}

\section{(1R,3a' R,6'R,6a'R)-6'-(benzyloxy)-2-chloro- 2',2'-dimethyldihydro-6'H-spiro[cyclobutane- 1,5'-furo[2,3-d][1,3]dioxol]-3-one (A)}

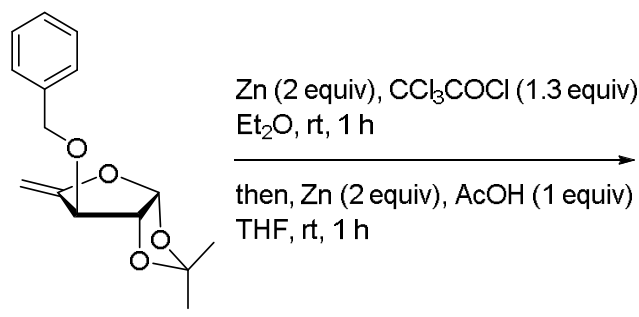

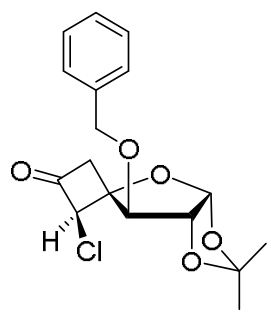

A.1

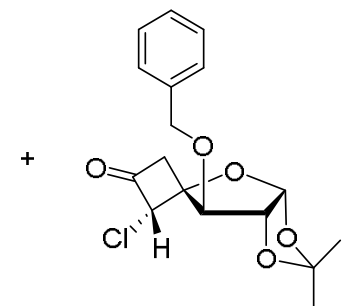

A.2

Compound 1,2-isopropylidene-3-0-benzyl-4-methylidene-L-threo furanose (75 g, $286 \mathrm{mmol}, 1$ equiv) was dissolved in $\mathrm{Et}_{2} \mathrm{O}(750 \mathrm{~mL})$ and zinc powder $(37.0 \mathrm{~g}, 569 \mathrm{mmol}, 2$ equiv) was added. This was followed by the addition of a solution of trichloroacetyl chloride $\left(70 \mathrm{~g}, 389 \mathrm{mmol}, 1.3\right.$ equiv) in $\mathrm{Et}_{2} \mathrm{O}$ $(200 \mathrm{~mL})$ dropwise with stirring at $0{ }^{\circ} \mathrm{C}$. The resulting solution was stirred for $1 \mathrm{~h}$ at room temperature. ${ }^{1 \mathrm{a}}$ Then zinc ( $92 \mathrm{~g}, 1.42 \mathrm{~mol}, 5$ equiv) was added in several portions, followed by $\mathrm{AcOH}$ (17 g, $283 \mathrm{mmol}, 1$ equiv) which was added dropwise while the solution was cooled with an ice bath to control the internal temperature below $10^{\circ} \mathrm{C}$. After addition, the mixture was stirred at room temperature for $10 \mathrm{~min}$. The residue was added to an aqueous saturated solution of $\mathrm{NaHCO}_{3}(1000$ $\mathrm{mL}$ ) at $0{ }^{\circ} \mathrm{C}$ then the mixture was filtered to remove the solids. The aqueous phase was extracted with EtOAc $(1000 \mathrm{~mL})$, the resulting organic layer was dried $\left(\mathrm{Na}_{2} \mathrm{SO}_{4}\right)$, filtered and the filtrate was concentrated under reduced pressure. The crude was purified over silica gel column chromatography (elution with PE/EtOAC $=99: 1$ to 83:17) to afford the product $\mathbf{A}(52 \mathrm{~g}, 153 \mathrm{mmol}, 54 \%)$ as a colorless oil and as a mixture of isomers A.1 and A.2 (A.1/A.2 = 86:14). Only the major isomer A.1 was described from the mixture.

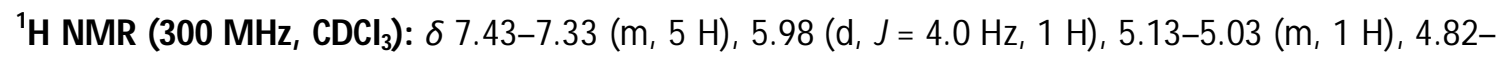
$4.75(\mathrm{~m}, 2 \mathrm{H}), 4.62(\mathrm{~d}, \mathrm{~J}=11.5 \mathrm{~Hz}, 1 \mathrm{H}), 4.45(\mathrm{~s}, 1 \mathrm{H}), 3.69-3.60(\mathrm{~m}, 1 \mathrm{H}), 3.32-3.24(\mathrm{~m}, 1 \mathrm{H}), 1.59(\mathrm{~s}, 3$ $\mathrm{H}), 1.40$ (s, $3 \mathrm{H}) \mathrm{ppm}$.

${ }^{13} \mathrm{C}\left\{{ }^{1} \mathrm{H}\right\}$ NMR (75 M Hz, CDCl$\left.{ }_{3}\right): \delta 197.7,136.8,128.7$ (2 C), 128.4, 127.9 (2 C), 113.3, 105.8, 84.3, 83.4, 82.0, 72.7, 69.2, 51.4, 26.8, 26.4 ppm. 

[2,3-d][1,3]dioxol]-2-yl)methanol (B)

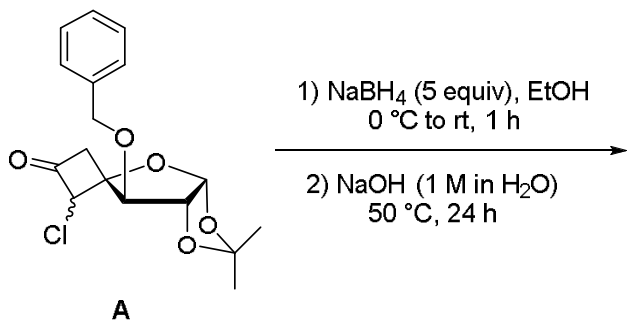

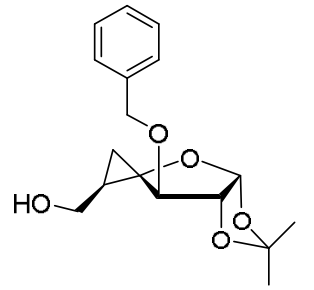

B.1

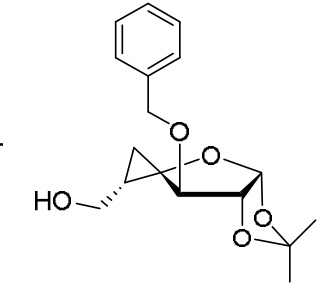

B.2

Compound A ( $2.2 \mathrm{~g}, 6.50 \mathrm{mmol}, 1$ equiv) was dissolved in EtOH ( $22 \mathrm{~mL})$, cooled to $0{ }^{\circ} \mathrm{C}$, and $\mathrm{NaBH}_{4}$ ( $1.23 \mathrm{~g}, 32.3 \mathrm{mmol}, 5$ equiv) was added at $0{ }^{\circ} \mathrm{C}$. The mixture was stirred at room temperature for $1 \mathrm{~h}$. Subsequently, $1 \mathrm{M}$ aqueous $\mathrm{NaOH}\left(88 \mathrm{~mL}\right.$ ) was added and stirring was continued at $50{ }^{\circ} \mathrm{C}$ for $24 \mathrm{~h}$. The product was extracted with EtOAc $(3 \times 50 \mathrm{~mL})$ and the combined organic layers were dried $\left(\mathrm{Na}_{2} \mathrm{SO}_{4}\right)$, filtered and the filtrate was concentrated under reduced pressure to afford the crude product $\mathbf{B}$ as a mixture of isomers $\mathbf{B} . \mathbf{1}$ and $\mathbf{B} . \mathbf{2}$ in a 82:18 ratio, respectively. The crude was purified by Preparative HPLC (Column: WelFlash C18-I Column, 50*250 mm 20u; Mobile Phase A: Water (10 mmoL/L $\mathrm{NH}_{4} \mathrm{HCO}_{3}$ ), Mobile Phase B: MeCN; Flow rate: $100 \mathrm{~mL} / \mathrm{min}$; Gradient: 40B to 70B in $30 \mathrm{~min}$; collection at $210 / 254 \mathrm{~nm})$ to afford $\mathbf{B . 1}(600 \mathrm{mg}, 1.96 \mathrm{mmol}, 30 \%)$ as a colorless oil and $\mathbf{B . 2}(80 \mathrm{mg}$, $0.26 \mathrm{mmol}, 4 \%$ ) as a colorless oil.

\section{B.1:}

${ }^{1}$ H NMR (300 MHz, DMSO-d d $_{6}: 8$ 7.36-7.34 (m, $\left.5 \mathrm{H}\right), 5.83(\mathrm{~d}, \mathrm{~J}=4.0 \mathrm{~Hz}, 1 \mathrm{H}), 4.78(\mathrm{~d}, \mathrm{~J}=4.0 \mathrm{~Hz}, 1 \mathrm{H})$, $4.65(\mathrm{~d}, \mathrm{~J}=11.9 \mathrm{~Hz}, 1 \mathrm{H}), 4.52(\mathrm{~d}, \mathrm{~J}=11.9 \mathrm{~Hz}, 1 \mathrm{H}), 4.38-4.34(\mathrm{~m}, 1 \mathrm{H}), 3.74(\mathrm{~s}, 1 \mathrm{H}), 3.50-3.46(\mathrm{~m}, 1$ H), 3.12-3.08 (m, $1 \mathrm{H}), 1.44(\mathrm{~s}, 3 \mathrm{H}), 1.27-1.24(\mathrm{~m}, 4 \mathrm{H}), 0.97-0.89(\mathrm{~m}, 1 \mathrm{H}), 0.69(\mathrm{t}, \mathrm{J}=6.7 \mathrm{~Hz}, 1 \mathrm{H})$ ppm.

$\left.{ }^{13} \mathrm{C}^{\mathrm{l}} \mathrm{H}\right\}$ NMR (75 MHz, DM SO-d 6 ): $\delta$ 138.0, 128.2 (2 C), 127.6 (2 C), 127.5, 111.1, 103.9, 83.1, 81.1, $70.2,68.9,60.5,26.31,26.28,25.4,9.0$ ppm.

LCMS (ESI): $\left[\mathrm{M}+\mathrm{NH}_{4}\right]^{+}=411,1.22 \mathrm{~min}$.

HRMS (ESI-TOF) m/ $\mathbf{z}$ : [M +Na] ${ }^{+}$Calcd for $\mathrm{C}_{17} \mathrm{H}_{22} \mathrm{O}_{5} \mathrm{Na} 329.1365$; Found 329.1363.

\section{B.2:}

${ }^{1}$ H NMR (300 MHz, DMSO-d N $): \delta 7.36-7.29(\mathrm{~m}, 5 \mathrm{H}), 5.91(\mathrm{~d}, \mathrm{~J}=4.2 \mathrm{~Hz}, 1 \mathrm{H}), 4.78(\mathrm{~d}, \mathrm{~J}=4.3 \mathrm{~Hz}, 1 \mathrm{H})$, $4.64(\mathrm{~d}, \mathrm{~J}=11.8 \mathrm{~Hz}, 1 \mathrm{H}), 4.47(\mathrm{~d}, \mathrm{~J}=11.8 \mathrm{~Hz}, 1 \mathrm{H}), 4.22(\mathrm{br} \mathrm{s}, 1 \mathrm{H}), 3.64-3.58(\mathrm{~m}, 2 \mathrm{H}), 3.21-3.15(\mathrm{~m}$, $1 \mathrm{H}), 1.43(\mathrm{~s}, 3 \mathrm{H}), 1.27(\mathrm{~s}, 3 \mathrm{H}), 1.24-1.14(\mathrm{~m}, 1 \mathrm{H}), 0.91-0.95(\mathrm{dd}, \mathrm{J}=9.4,6.6 \mathrm{~Hz}, 1 \mathrm{H}), 0.66(\mathrm{t}, \mathrm{J}=6.6$ $\mathrm{Hz}, 1 \mathrm{H}) \mathrm{ppm}$.

$\left.{ }^{13} \mathrm{C}^{1}{ }^{1} \mathrm{H}\right\}$ NMR (75 MHz, DM SO-d $\left.\mathbf{d}_{6}\right) ; \delta 138.6,128.7$ (2 C), 128.1 (2 C), 128.0, 111.0, 105.1, 86.5, 83.0, $70.74,70.69,60.6,26.6,26.2,25.3,9.4 \mathrm{ppm}$.

HRMS (ESI-TOF) m/ z: [M +Na] ${ }^{+}$Calcd for $\mathrm{C}_{17} \mathrm{H}_{22} \mathrm{O}_{5} \mathrm{Na}$ 329.1365; Found 329.1365. 


\section{Optimization tables of the stereoselective cyclopropanation}

\section{* Using (S)-Ph-Pheox Ru(II):}

In an oven dried round-bottom flask under argon atmosphere, substrate 4-exo-methylene furanoside 1 ( $0.5 \mathrm{mmol}, 1$ equiv) was dissolved in dry solvent (concentration in the table) and catalyst (S)Ph-Pheox Ru(II) (mol \% in the table) was added. A solution of ethyl diazoacetate (EDA) in the same dry solvent as previously (equiv and concentration in the table) was added dropwise at the temperature indicated in the table. The resulting mixture was stirred at the temperature indicated in the table. TLC monitoring was performed to follow the reaction (eluant PE/EtOAC $=90: 10$ ). The catalyst was filtered over a celite pad and the filtrate was concentrated under reduced pressure. The analysis of the ${ }^{1} \mathrm{H}$ NMR spectrum of the crude mixture containing trimethoxybenzene as an internal standard allowed to determine the conversion of the substrate, the yield of the major product and the diastereomeric ratio.

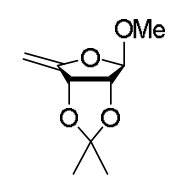

1

\begin{tabular}{|c|c|c|c|c|c|c|c|c|}
\hline Entry & Solvent & $\begin{array}{c}\mathbf{C} \mathbf{1} \\
\text { before } \\
\text { adding } \\
\text { EDA }\end{array}$ & $\begin{array}{c}\text { EDA } \\
\text { (equiv) } \\
\text { CEDA }\end{array}$ & $\begin{array}{c}\text { (S)-Ph- } \\
\text { Pheox-Ru(II) } \\
\text { (mol \%) }\end{array}$ & $\begin{array}{c}\mathbf{T} \\
\mathbf{t}(\mathbf{h})\end{array}$ & $\begin{array}{c}\text { Substrate } \\
\text { conversion }\end{array}$ & $\begin{array}{c}\text { Crude dr } \\
\left(\beta^{\prime} \text {-trans/ }\right. \\
\alpha^{\prime} \text {-cis/ } \beta \text {-cis) }\end{array}$ & $\begin{array}{c}\text { NMR Yield } \\
\text { of } \\
\beta^{\prime} \text {-trans-2 }\end{array}$ \\
\hline $\mathbf{1}$ & $\mathrm{CH}_{2} \mathrm{Cl}_{2}$ & $0.50 \mathrm{M}$ & $\begin{array}{c}1.5 \\
0.44 \mathrm{M}\end{array}$ & 6 & $\begin{array}{c}\text { rt } \\
24\end{array}$ & $65 \%$ & $84: 12: 4$ & $37 \%$ \\
\hline $\mathbf{2}$ & $\mathrm{CH}_{2} \mathrm{Cl}_{2}$ & $0.40 \mathrm{M}$ & $\begin{array}{c}1.5 \\
0.33 \mathrm{M}\end{array}$ & 6 & $\begin{array}{c}\text { rt } \\
2\end{array}$ & $59 \%$ & $85: 11: 4$ & $33 \%$ \\
\hline $\mathbf{3}$ & $\mathrm{CH}_{2} \mathrm{Cl}_{2}$ & $0.50 \mathrm{M}$ & $\begin{array}{c}3 \\
0.44 \mathrm{M}\end{array}$ & 6 & $\begin{array}{c}\mathrm{rt} \\
3\end{array}$ & $91 \%$ & $85: 11: 4$ & $59 \%$ \\
\hline $\mathbf{4}$ & $\mathrm{CH}_{2} \mathrm{Cl}_{2}$ & $0.50 \mathrm{M}$ & $\begin{array}{c}1.5 \\
0.44 \mathrm{M}\end{array}$ & 8 & $\begin{array}{c}\mathrm{rt} \\
3\end{array}$ & $64 \%$ & $85: 11: 4$ & $36 \%$ \\
\hline $\mathbf{5}$ & $\mathrm{CH}_{2} \mathrm{Cl}_{2}$ & $0.50 \mathrm{M}$ & $\begin{array}{c}4 \\
0.44 \mathrm{M}\end{array}$ & 6 & $\begin{array}{c}\mathrm{rt} \\
3\end{array}$ & $78 \%$ & $85: 11: 4$ & $51 \%$ \\
\hline $\mathbf{6}$ & $\mathrm{CH}_{3} \mathrm{CN}$ & $0.50 \mathrm{M}$ & $\begin{array}{c}3 \\
0.44 \mathrm{M}\end{array}$ & 6 & $\begin{array}{c}\mathrm{rt} \\
3\end{array}$ & $50 \%$ & $88: 8: 4$ & $28 \%$ \\
\hline $\mathbf{7}$ & $\mathrm{CH}_{2} \mathrm{Cl}_{2}$ & $0.50 \mathrm{M}$ & $\begin{array}{c}3 \\
0.44 \mathrm{M}\end{array}$ & 6 & $\begin{array}{c}0^{\circ} \mathrm{C} \\
2.5\end{array}$ & $100 \%$ & $87: 10: 3$ & $70 \%$ \\
\hline $\mathbf{8}$ & $\mathrm{CH}_{2} \mathrm{Cl}_{2}$ & $0.50 \mathrm{M}$ & $\begin{array}{c}3 \\
0.44 \mathrm{M}\end{array}$ & 6 & $\begin{array}{c}-20^{\circ} \mathrm{C} \\
5\end{array}$ & $76 \%$ & $90: 8: 2$ & $53 \%$ \\
\hline $\mathbf{9}$ & $\mathrm{CH}_{2} \mathrm{Cl}_{2}$ & $0.50 \mathrm{M}$ & $\begin{array}{c}3 \\
0.44 \mathrm{M}\end{array}$ & 6 & $\begin{array}{c}0^{\circ} \mathrm{C} \\
2^{\mathrm{a}}\end{array}$ & $100 \%$ & $88: 10: 2$ & $75 \%$ \\
\hline $\mathbf{1 0}$ & $\mathrm{CH}_{2} \mathrm{Cl}_{2}$ & $0.50 \mathrm{M}$ & $\begin{array}{c}3 \\
0.44 \mathrm{M}\end{array}$ & 6 & $\begin{array}{c}-10^{\circ} \mathrm{C} \\
2\end{array}$ & $100 \%$ & $89: 9: 2$ & $70 \%$ \\
\hline $\mathbf{1 1}$ & $\mathrm{CH}_{2} \mathrm{Cl}_{2}$ & $0.50 \mathrm{M}$ & $\begin{array}{c}3 \\
0.44 \mathrm{M}\end{array}$ & 6 & $\begin{array}{c}-10^{\circ} \mathrm{C} \\
2^{\mathrm{a}}\end{array}$ & $100 \%$ & $89: 9: 2$ & $72 \%$ \\
\hline
\end{tabular}

a Slow addition of EDA solution over $2 \mathrm{~h}$ using a syringe pump. ${ }^{b}$ Compound $\beta^{\prime}$-trans-2 was isolated in $63 \%$ yield. $\underset{\text { solvent, T, } 2-24 \mathrm{~h}}{\stackrel{(S)-P h \text {-Pheox Ru(II) }(y \mathrm{~mol} \%)}{\longrightarrow}}$

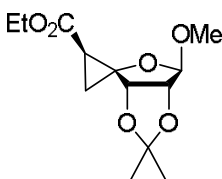

$\beta$ trans-2

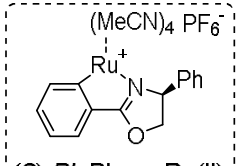

(S)-Ph-Pheox Ru(II)

Table S1: Optimization of the stereoselective cyclopropanation using (S)-Ph-Pheox Ru(II) 


\section{* Using (R)-Ph-Pheox Ru(II):}

In an oven dried round-bottom flask under argon atmosphere, substrate 4-exo-methylene furanoside $1\left(0.5 \mathrm{mmol}, 1\right.$ equiv) was dissolved in dry $\mathrm{CH}_{2} \mathrm{Cl}_{2}$ (concentration in the table) and catalyst (R)-Ph-Pheox Ru(II) (6 mol \%) was added. A solution of ethyl diazoacetate (EDA) in dry $\mathrm{CH}_{2} \mathrm{Cl}_{2}$ (equiv and concentration in the table) was added dropwise at the temperature indicated in the table. The resulting mixture was stirred at room temperature (or $0{ }^{\circ} \mathrm{C}$ when indicated). TLC monitoring was performed to follow the reaction (eluant PE/EtOAC $=90: 10$ ). The catalyst was filtered over a celite pad and the filtrate was concentrated under reduced pressure. The analysis of the ${ }^{1} \mathrm{H}$ NMR spectrum of the crude mixture containing trimethoxybenzene as an internal standard allowed to determine the conversion of the substrate, the yield of the major product and the diastereomeric ratio.

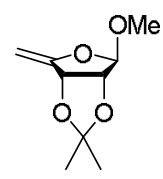

1

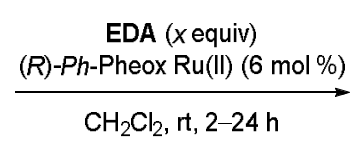

$\mathrm{CH}_{2} \mathrm{Cl}_{2}, \mathrm{rt}, 2-24 \mathrm{~h}$

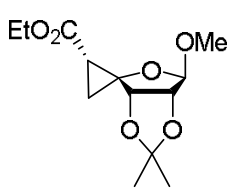

$\beta$-cis-2

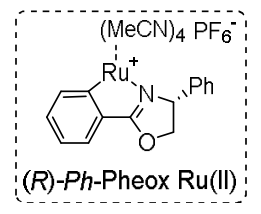

(R)-Ph-Pheox Ru(II)

\begin{tabular}{|c|c|c|c|c|c|c|c|}
\hline Entry & $\begin{array}{c}\mathbf{C}_{\mathbf{1}} \text { before } \\
\text { adding } \\
\text { EDA }\end{array}$ & $\begin{array}{c}\text { EDA } \\
\text { (equiv) } \\
\mathbf{C}_{\text {EDA }}\end{array}$ & $\mathbf{t}$ (h) & $\mathbf{T}$ & $\begin{array}{c}\text { Substrate } \\
\text { conversion }\end{array}$ & $\begin{array}{c}\text { Crude dr } \\
\left(\beta^{\prime} \text {-cis/ } \alpha^{\prime} \text {-trans/ } \beta \text {-trans) }\right.\end{array}$ & $\begin{array}{c}\text { NMR Yield } \\
\text { of } \beta^{\prime} \text {-cis-2 }\end{array}$ \\
\hline $\mathbf{1}$ & $0.50 \mathrm{M}$ & $\begin{array}{c}1.5 \\
0.44 \mathrm{M}\end{array}$ & 24 & $\mathrm{rt}$ & $74 \%$ & $91: 8: 1$ & $48 \%$ \\
\hline $\mathbf{2}$ & $0.40 \mathrm{M}$ & $\begin{array}{c}1.5 \\
0.33 \mathrm{M}\end{array}$ & 2 & $\mathrm{rt}$ & $80 \%$ & $91: 8: 1$ & $58 \%$ \\
\hline $\mathbf{3}$ & $0.40 \mathrm{M}$ & $\begin{array}{c}3 \\
0.33 \mathrm{M}\end{array}$ & 3 & $\mathrm{rt}$ & $100 \%$ & $91: 8: 1$ & $79 \%$ \\
\hline $\mathbf{4}$ & $0.40 \mathrm{M}$ & $\begin{array}{c}3 \\
0.33 \mathrm{M}\end{array}$ & 2.5 & $0^{\circ} \mathrm{C}$ & $100 \%$ & $92: 7: 1$ & $80 \%$ \\
\hline $\mathbf{5}$ & $0.40 \mathrm{M}$ & $\begin{array}{c}3 \\
0.33 \mathrm{M}\end{array}$ & $2^{\mathrm{a}}$ & $\mathrm{rt}$ & $100 \%$ & $92: 7: 1$ & $81 \%^{\mathrm{b}}$ \\
\hline
\end{tabular}

a Slow addition of EDA solution over $2 \mathrm{~h}$ using a syringe pump. ${ }^{\mathrm{b}}$ Compound $\beta^{\prime}$-cis-2 was isolated in $74 \%$ yield.

Table S2: Optimization of the stereoselective cyclopropanation using (R)-Ph-Pheox Ru(II)

Note: The optimization of the reaction conditions for both catalysts was done in parallel. While using (R)-Ph-Pheox-Ru(II), we obtained the best compromise at room temperature, in terms of substrate conversion, diastereomeric ratio and product yield. Lowering the temperature to $0{ }^{\circ} \mathrm{C}$ gave similar results, so the interactions between the catalyst and the substrate does not seem to be influenced by the reaction temperature. However, with (S)-Ph-Pheox-Ru(II), lowering the temperature allowed us to achieve optimal results. The difference in the reaction temperatures when using (S)-Ph-Pheox$\mathrm{Ru}(\mathrm{II})$ and (R)-Ph-Pheox-Ru(II) could lie in the stability of (S)-Ph-Pheox-Ru(II). 


\section{Biological studies: Enzyme inhibition assay of PRM T5-MEP50 complex}

Compounds 10-13 and adenosine were assayed as inhibitors of PRM T5-MEP50 complex using a filter based radioactivity assay (SPA) as part of a commercially available panel of human Arg and Lys methyltransferases (Eurofins, https://www.eurofinsdiscoveryservices.com/services/in-vitroassays/epigenetics). Assay information is described on the Eurofins website (https:// www.eurofinsdiscoveryservices.com/catalogmanagement/viewitem/ PRM T5-ComplexHuman-Protein-Arginine-N-M ethyltransferase-Enzymatic-Assay-Cerep/3568).

The test compounds were assayed in an 8 point dose-response, up to $100 \mu \mathrm{M}$ top concentration $(100,30,10,3,1,0.3,0.1,0.03 \mu \mathrm{M})$, in 3 independent replicate experiments. $I_{50}$ values were calculated from the competition curves and expressed in $\mu \mathrm{M}$.

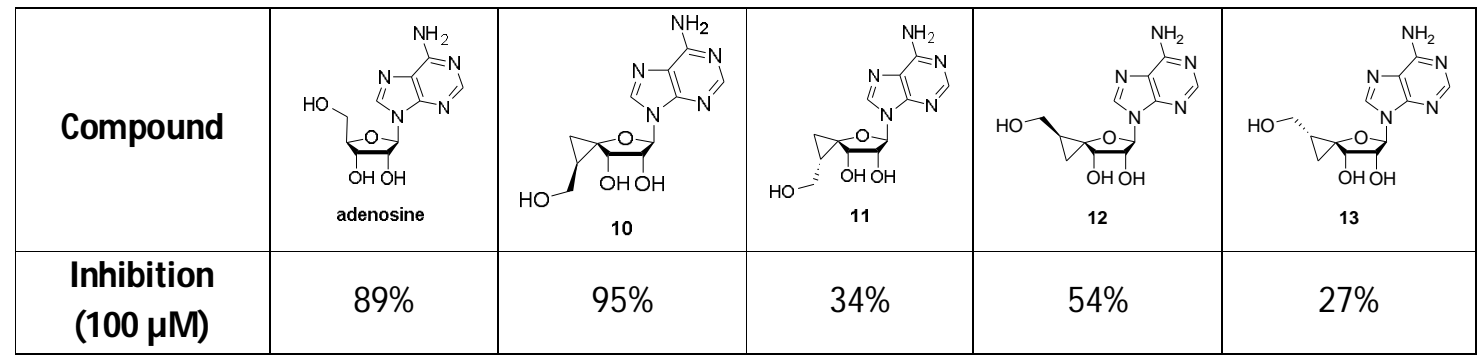

Table S3: Results of biological studies for adenosine and compounds 10-13. 


\section{Copies of NM R spectra}

\section{${ }^{1} \mathrm{H}$ NMR, $300 \mathrm{MHz}, \mathrm{CDCl}_{3}$ (contains $14 \%$ of A.2)}

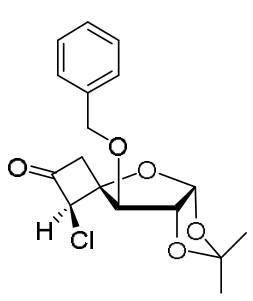

A.1

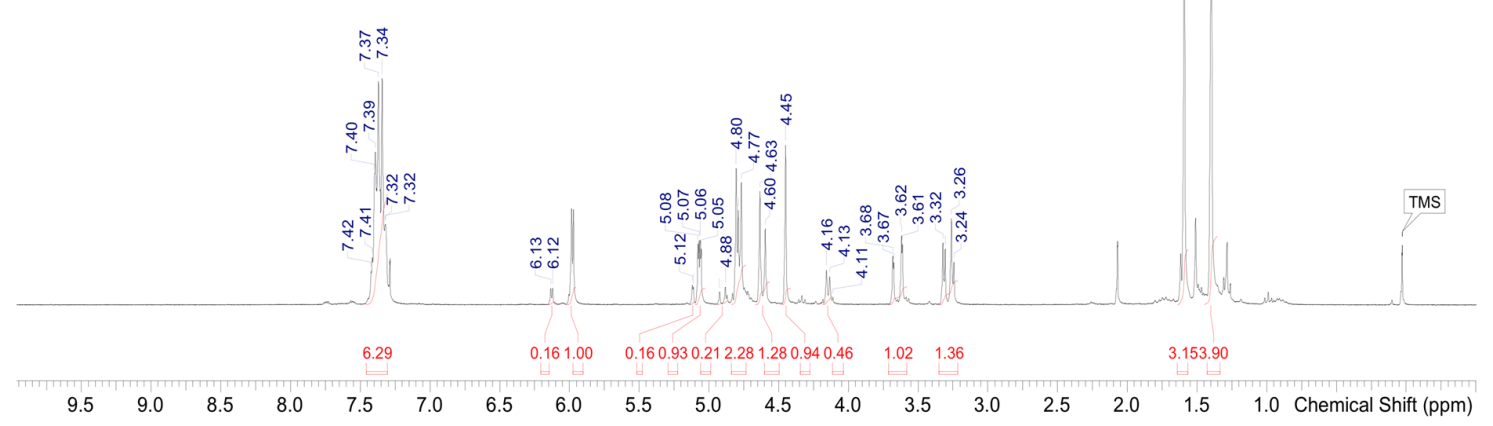

\section{$\left.{ }^{13} \mathrm{C}_{\{}{ }^{\mathrm{l}} \mathrm{H}\right\} \mathrm{NMR}, 75 \mathrm{M} \mathrm{Hz}, \mathrm{CDCl}_{3}$ (contains $14 \%$ of A.2)}
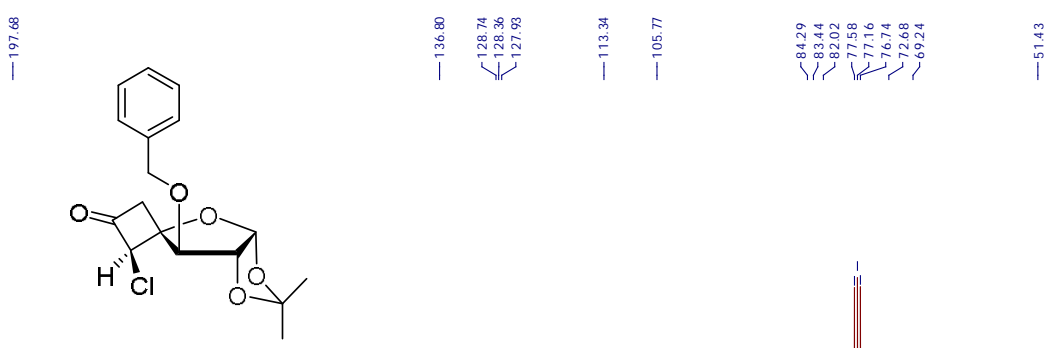

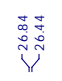

A.1

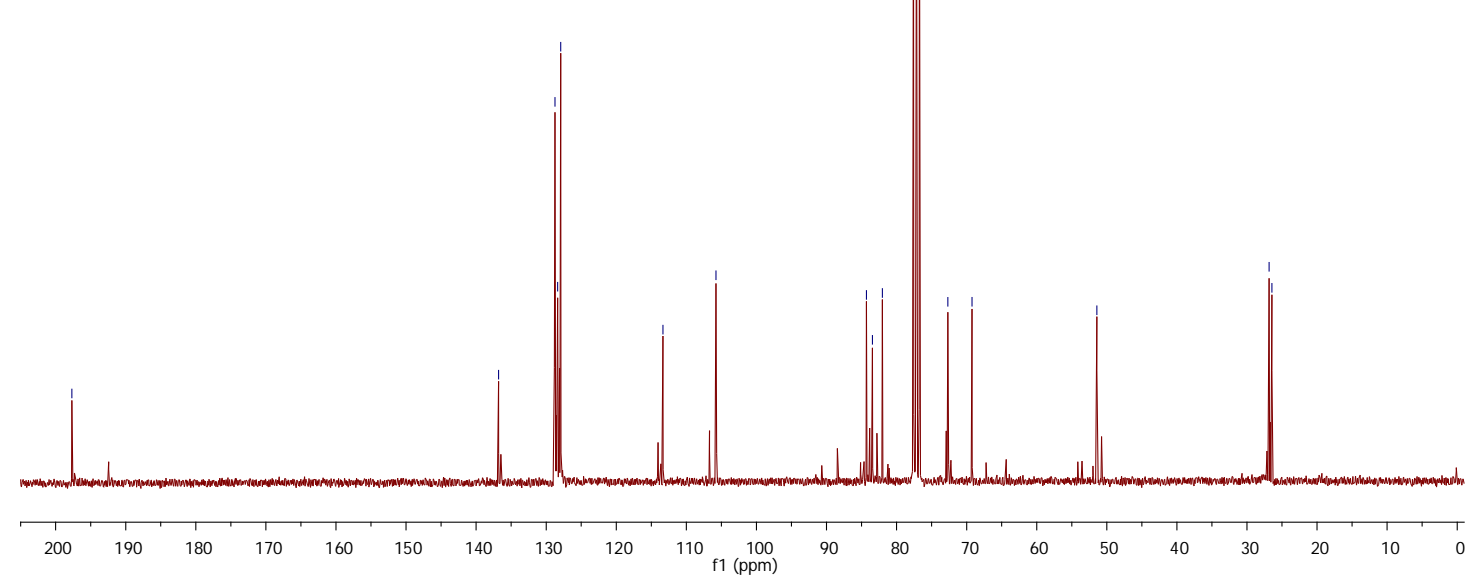


${ }^{1}$ H NMR, 300 MHz, DMSO-d 6

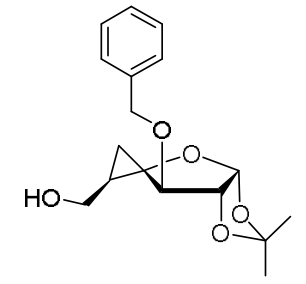

B.1

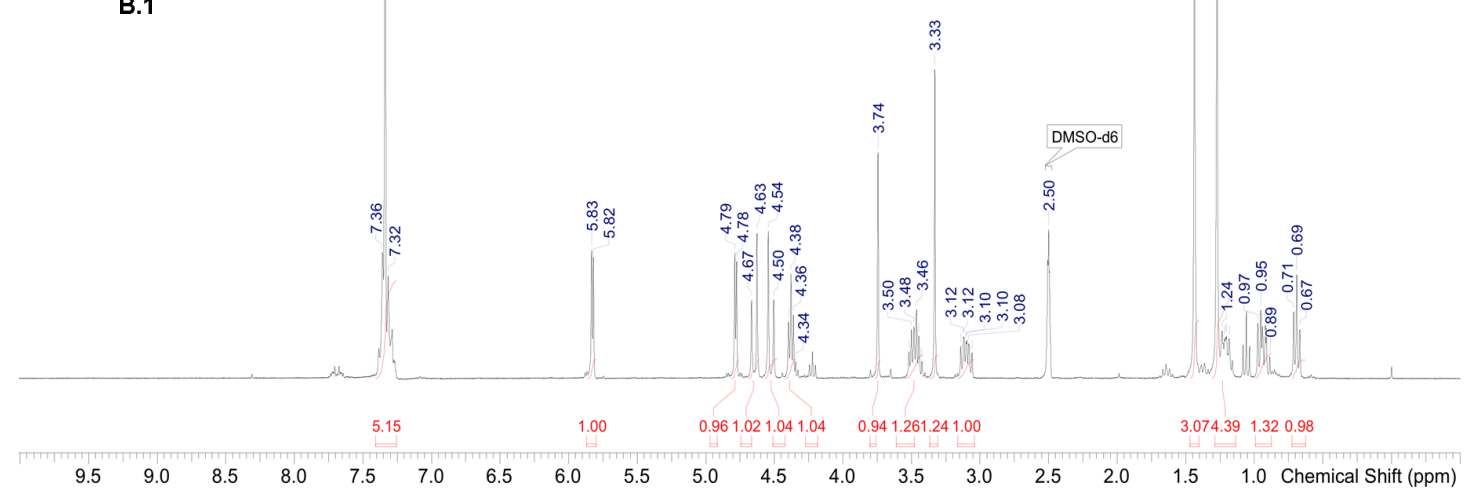

\section{${ }^{13} \mathrm{C}\left\{{ }^{\mathrm{l}} \mathrm{H}\right\}$ NMR, $75 \mathrm{M} \mathrm{Hz}, \mathrm{DMSO}-\mathrm{d}_{6}$}

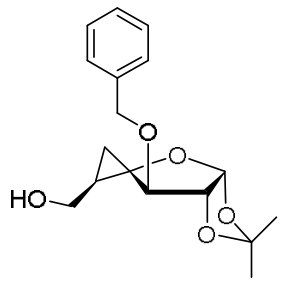

B.1

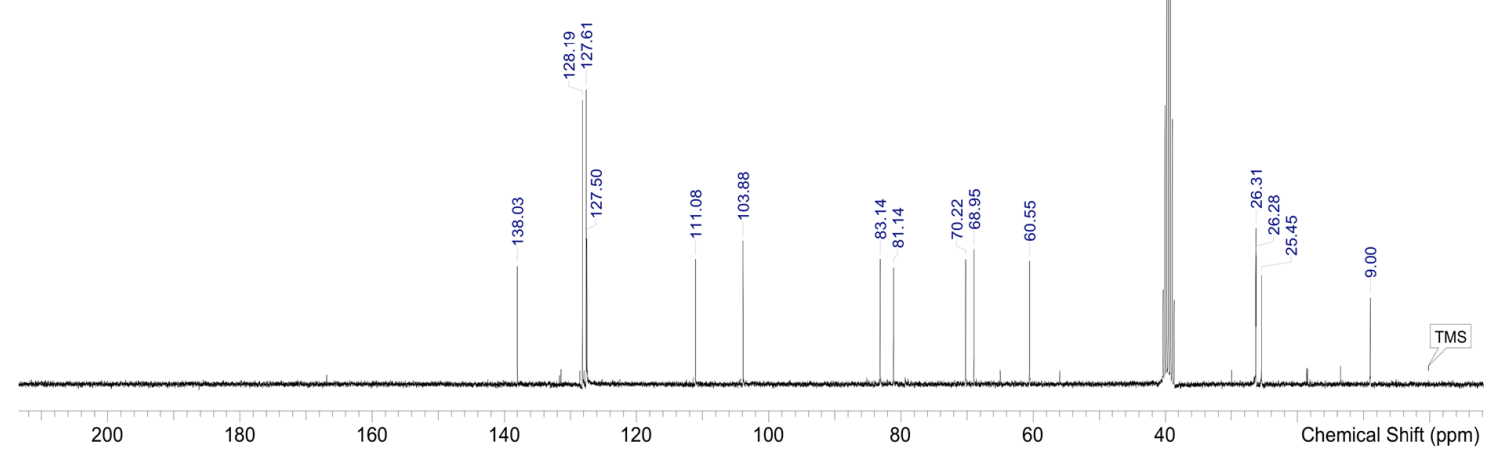


${ }^{1}$ H NMR, 300 MHz, DMSO-d 6

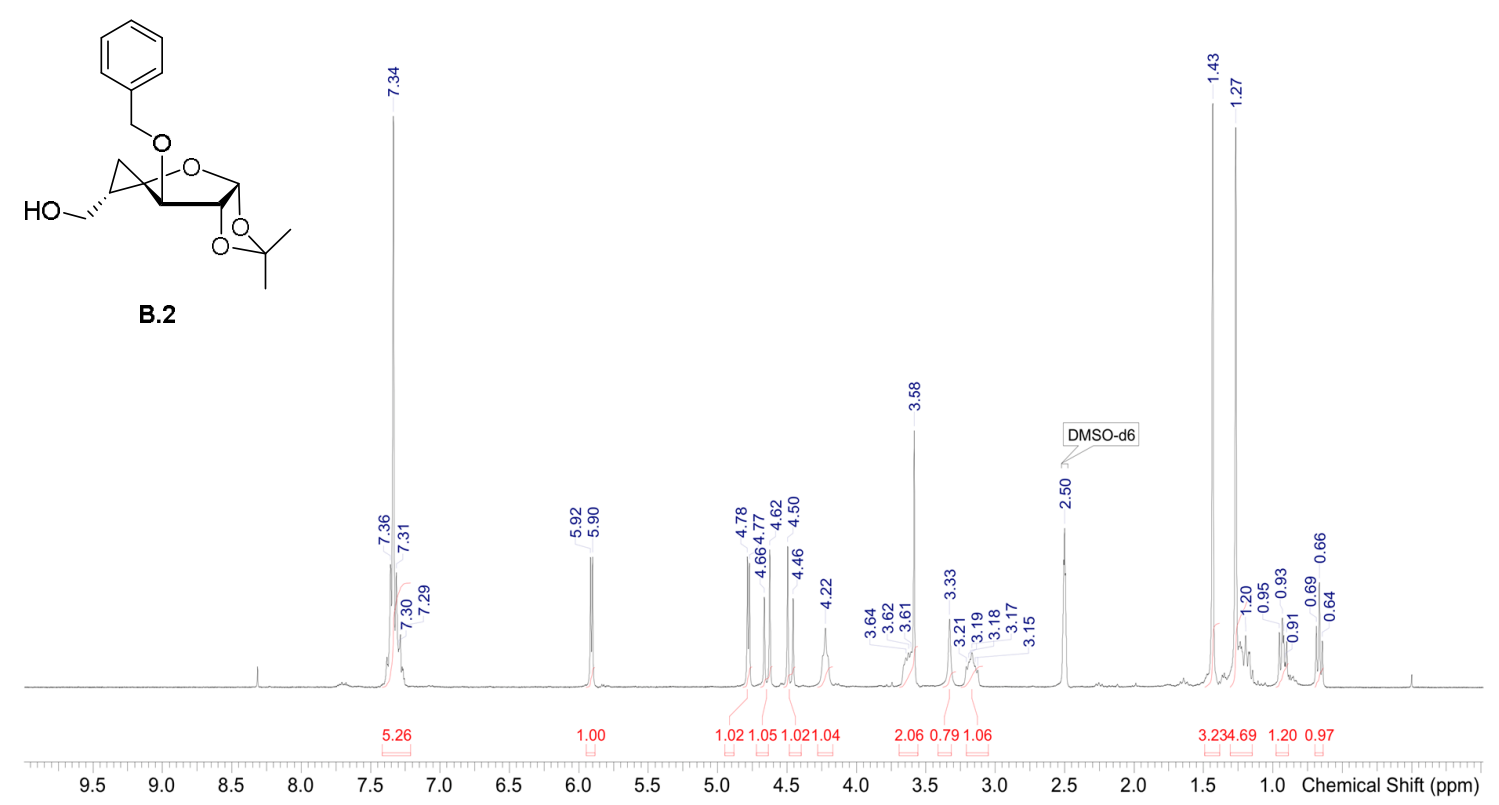

${ }^{13} \mathrm{C}\left\{{ }^{1} \mathrm{H}\right\}$ NMR, 75 M Hz, DM SO-d 6

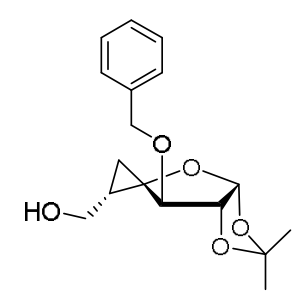

B.2

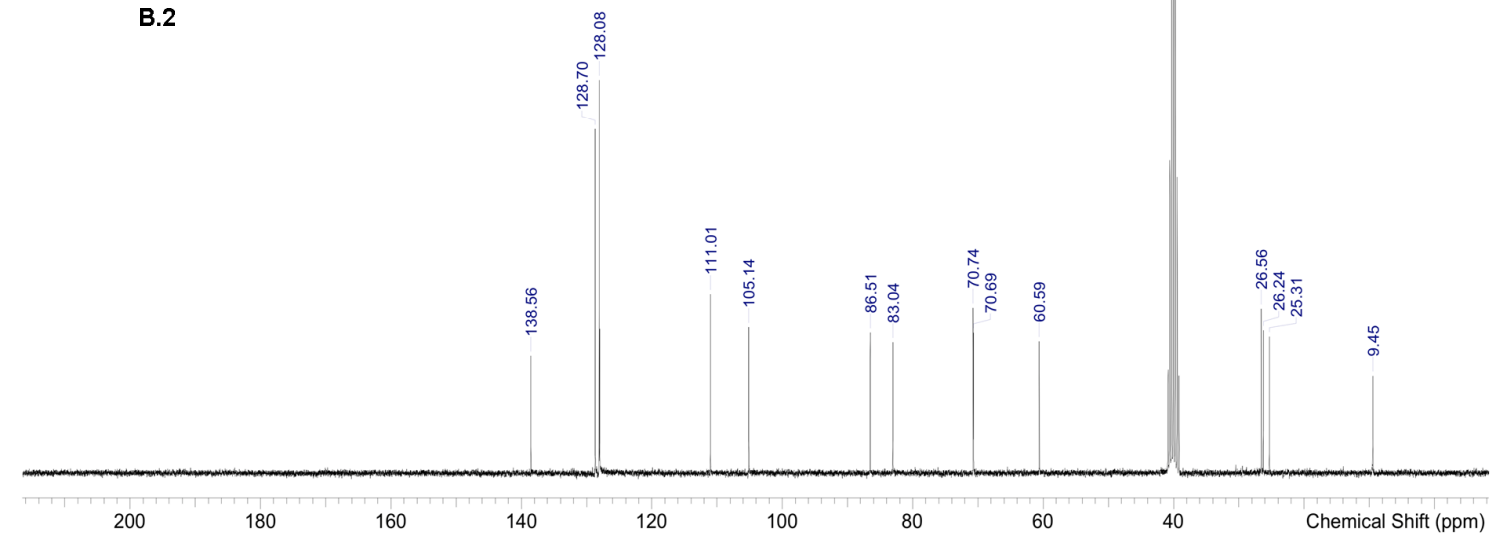


${ }^{1} \mathrm{H}$ NMR, $400 \mathrm{MHz}, \mathrm{CDCl}_{3}$

管

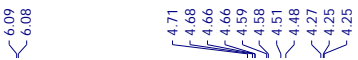

里赵,

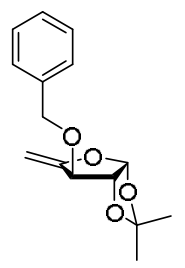

4a

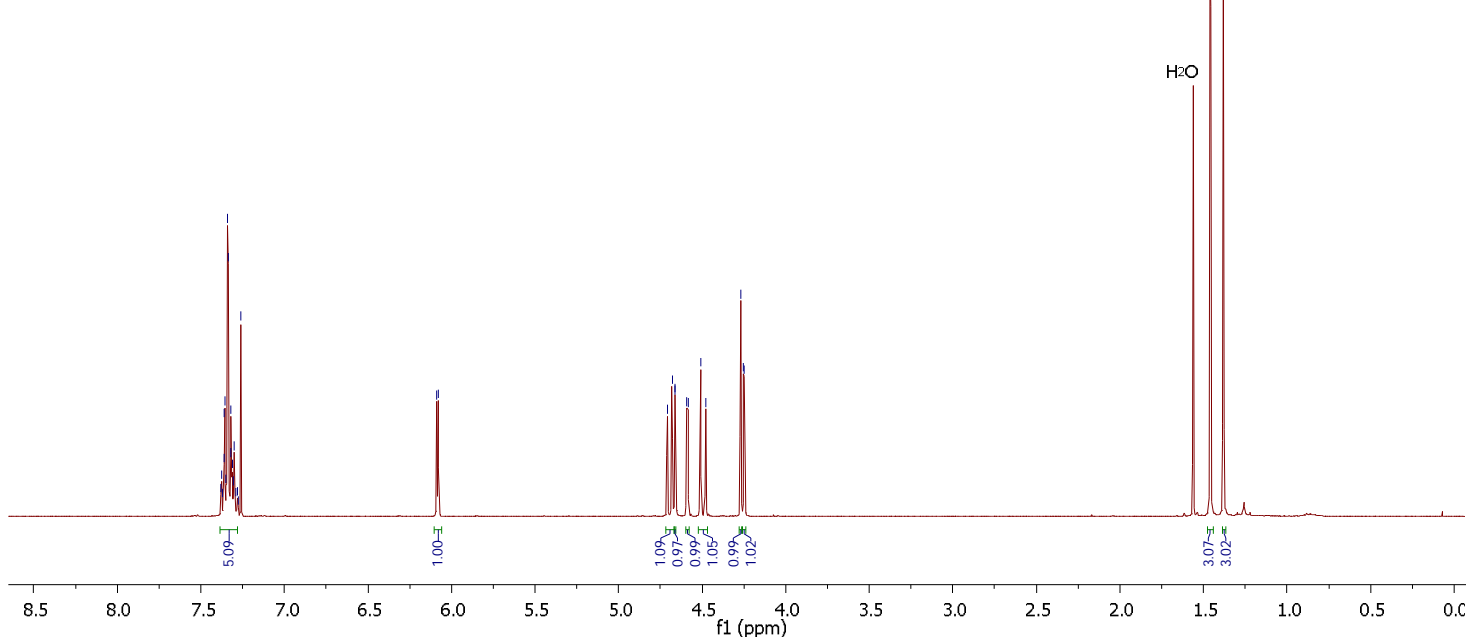

$\left.{ }^{13} \mathrm{C}_{\{}{ }^{1} \mathrm{H}\right\} \mathrm{NMR}, 100 \mathrm{M} \mathrm{Hz}, \mathrm{CDCl}_{3}$

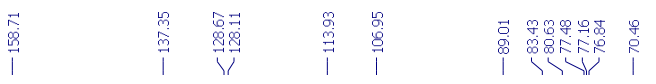

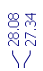

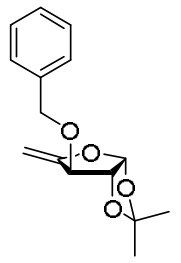

4a

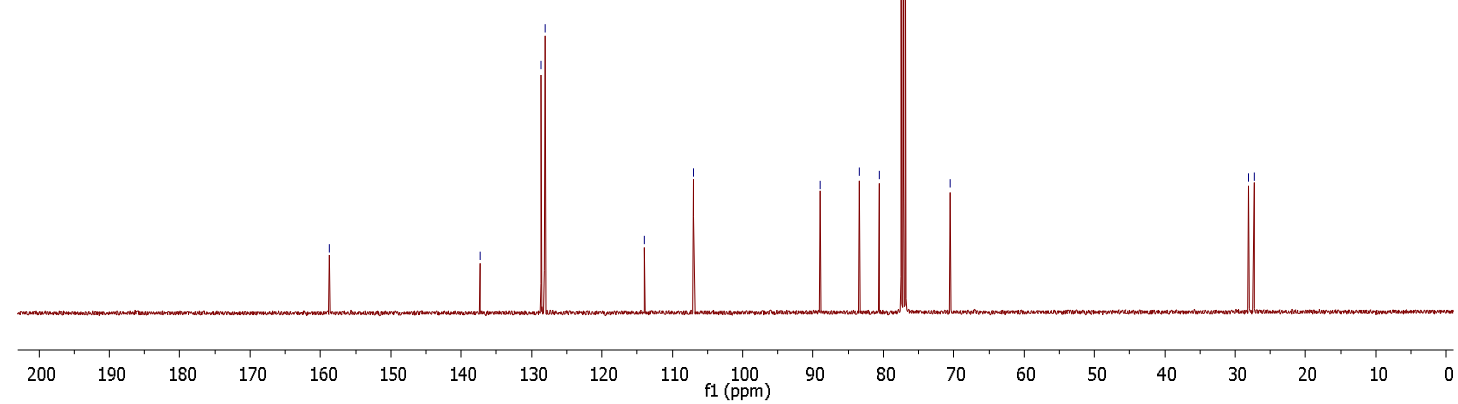

S-11 
${ }^{1} \mathrm{H}$ NMR, $400 \mathrm{MHz}, \mathrm{CDCl}_{3}$

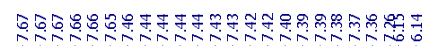

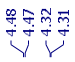

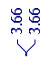

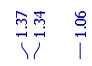

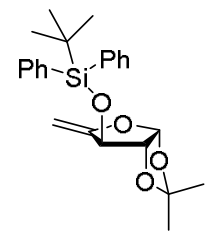

4b

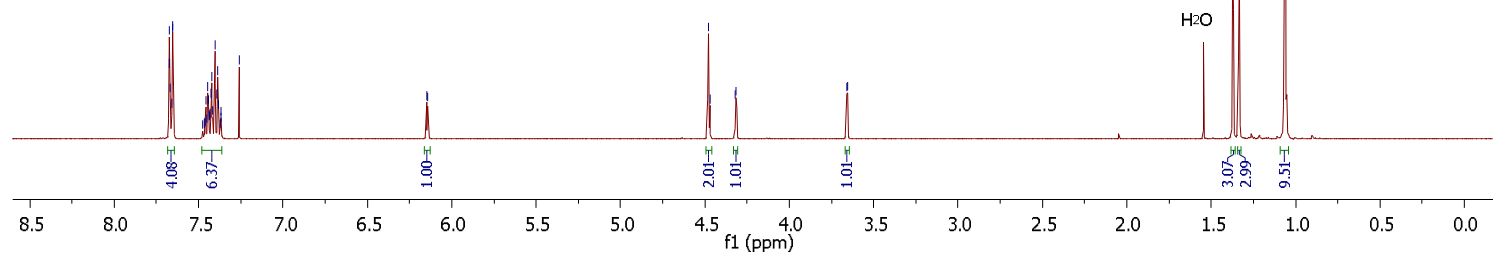

$\left.{ }^{13} \mathrm{C}_{\{}^{\mathrm{l}} \mathrm{H}\right\} \mathrm{NMR}, 100 \mathrm{MHz}, \mathrm{CDCl}_{3}$
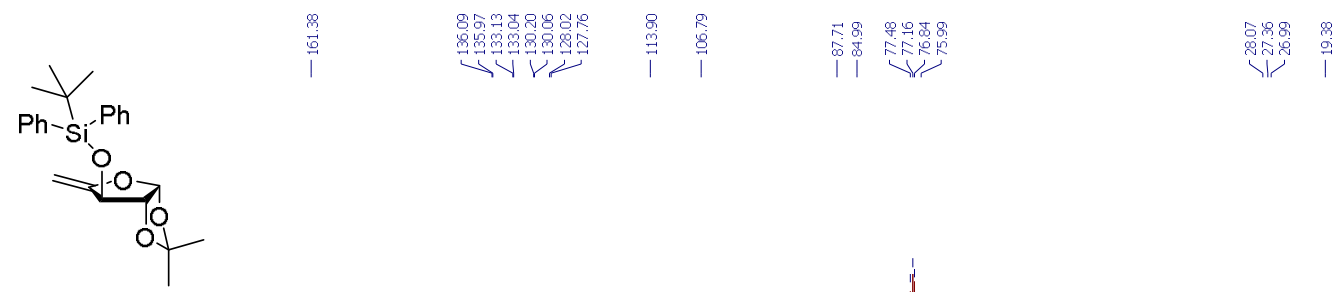

4b

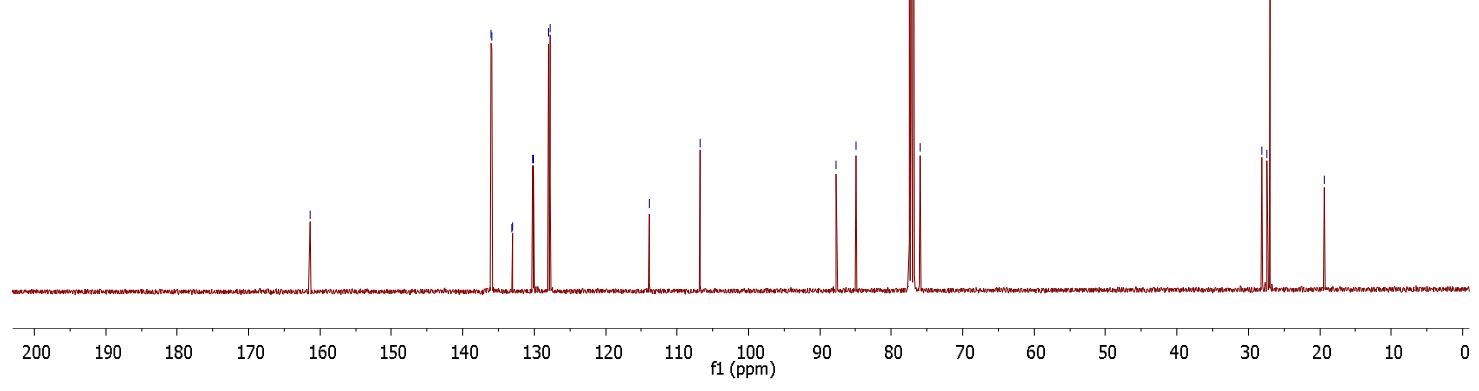

S-12 
${ }^{1} \mathrm{H}$ NMR, $400 \mathrm{MHz}, \mathrm{CDCl}_{3}$

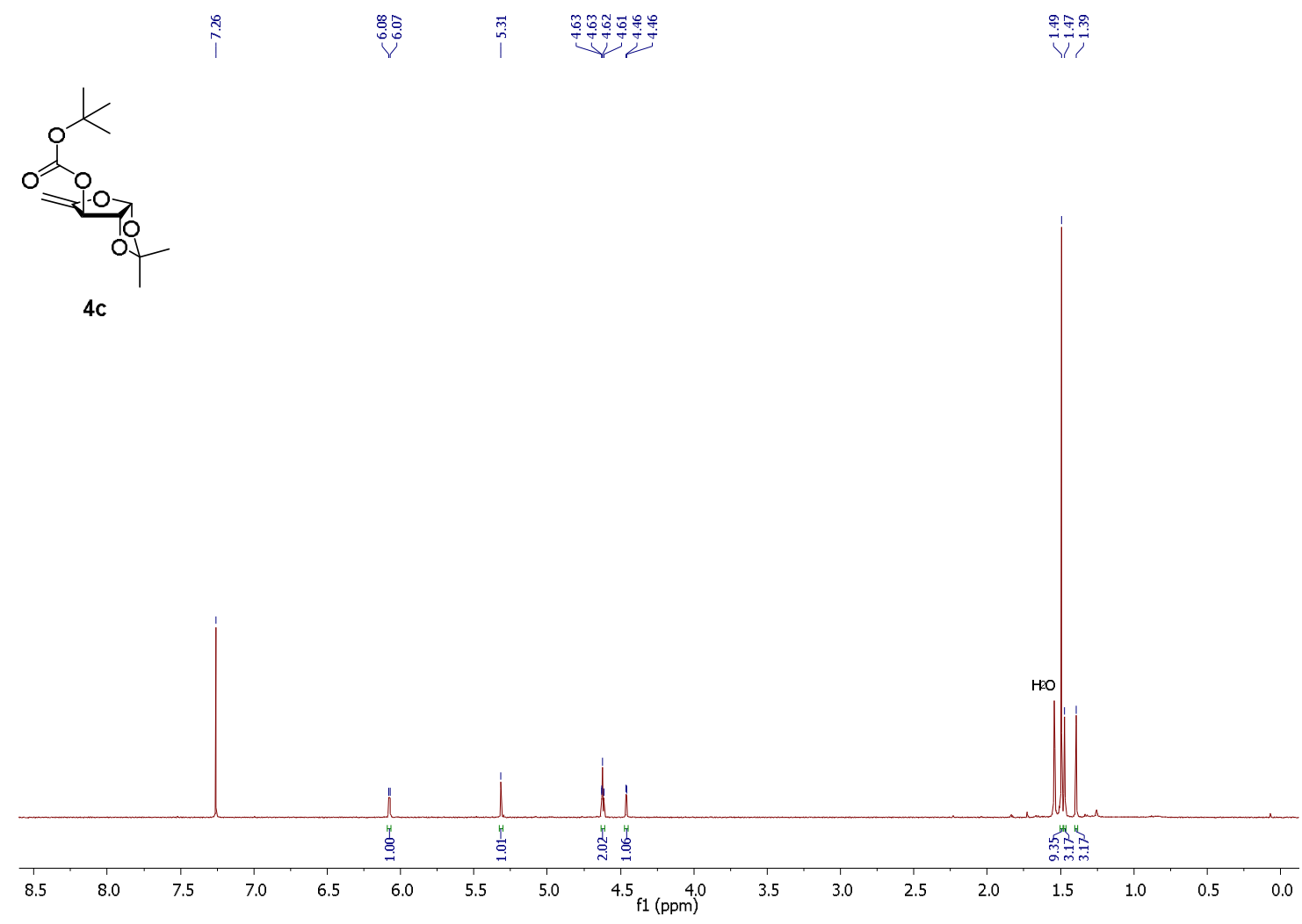

${ }^{13} \mathrm{C}\left\{{ }^{-} \mathrm{H}\right\} \mathrm{NMR}, 100 \mathrm{M} \mathrm{Hz}, \mathrm{CDCl}_{3}$
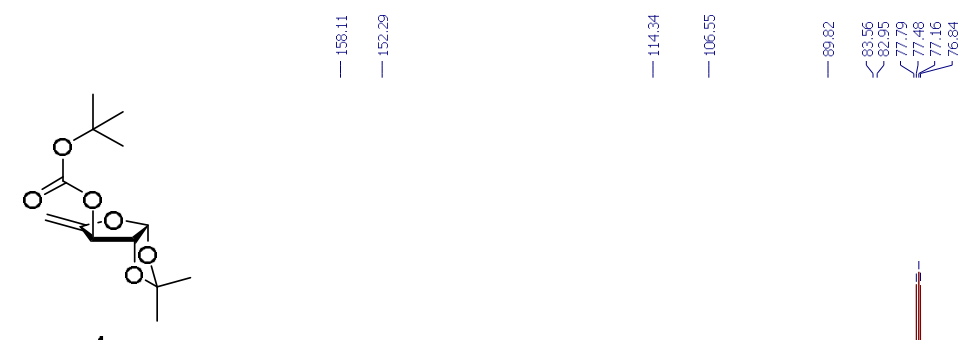

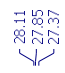

4c

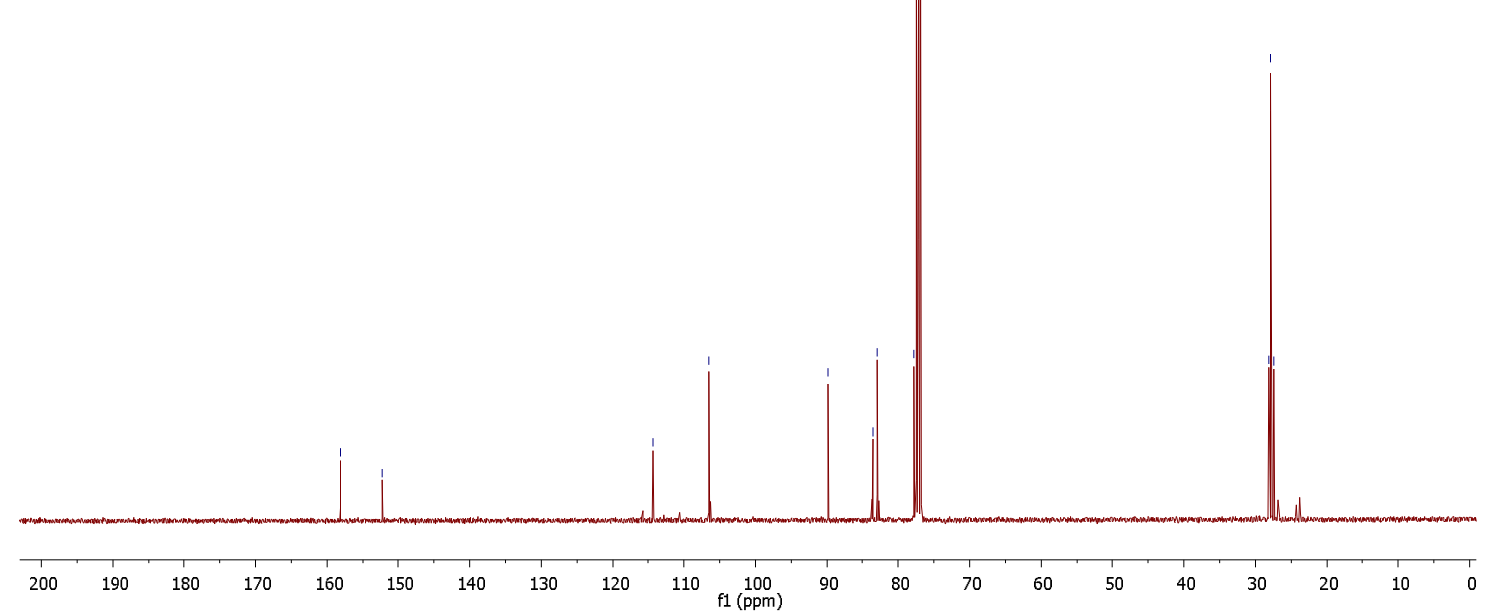

S-13 
${ }^{1} \mathrm{H} N \mathrm{NM}, 300 \mathrm{MHz}, \mathrm{CD}_{2} \mathrm{Cl}_{2}$

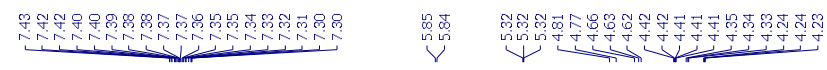<smiles>CC1(C)OC2OC(COc3ccccc3)C(O2)O1</smiles>

$4 d$

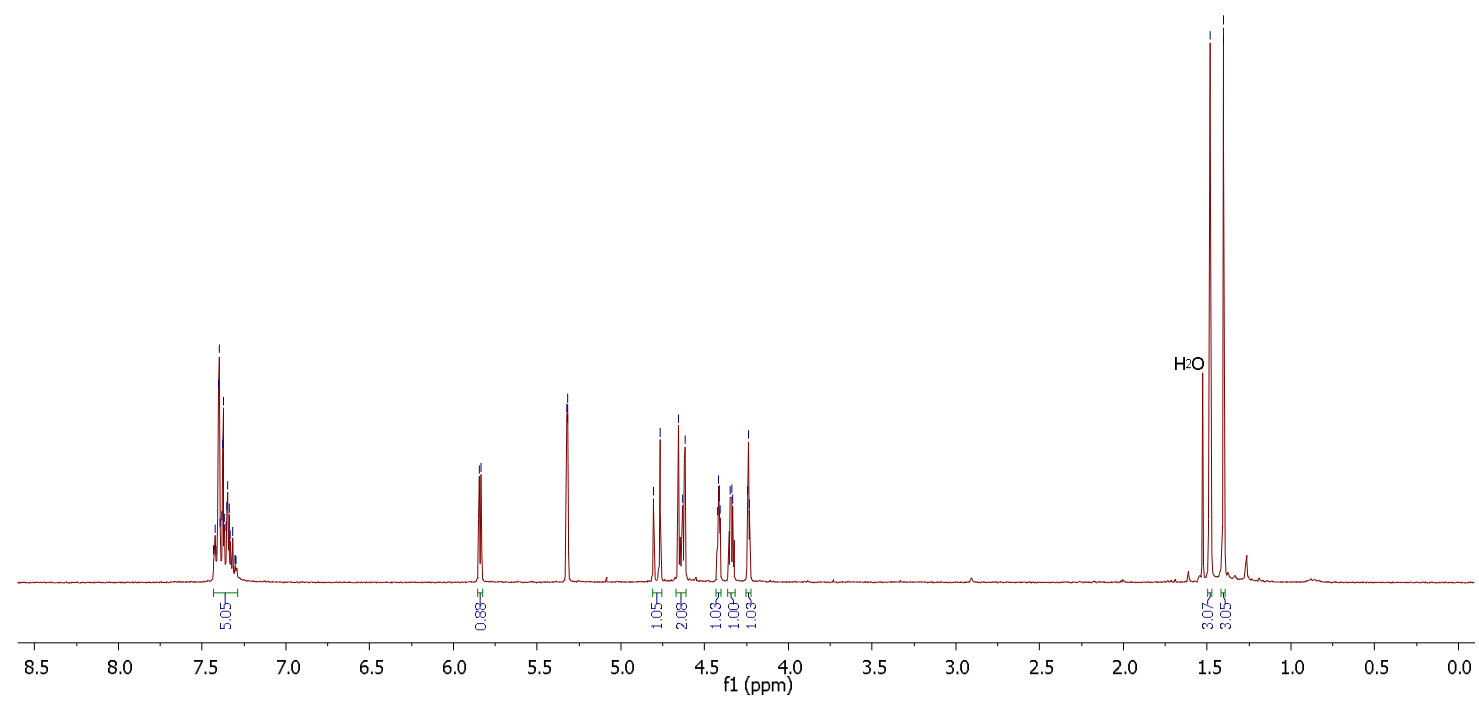

${ }^{13} \mathrm{C}\{\mathrm{l} H\} N M R, 75 \mathrm{MHz}, \mathrm{CD}_{2} \mathrm{Cl}_{2}$
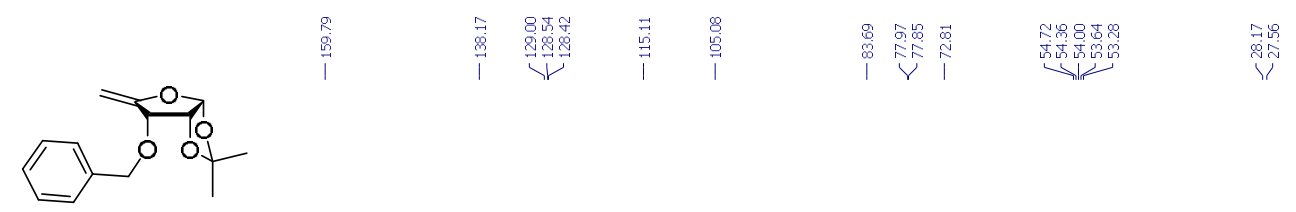

$4 d$

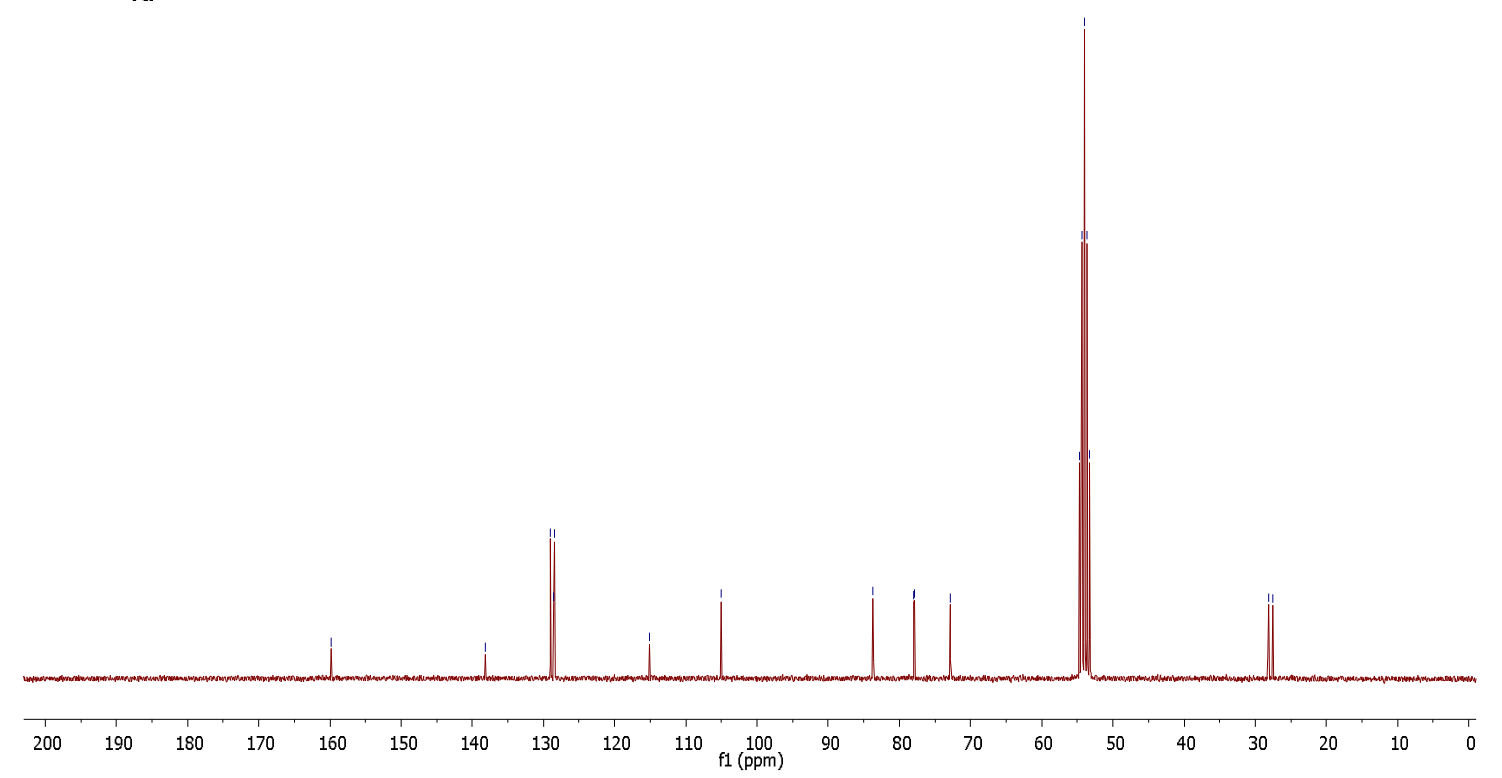

S-14 


\section{${ }^{1} \mathrm{H}$ NMR, $400 \mathrm{MHz}, \mathrm{CD}_{2} \mathrm{Cl}_{2}$}

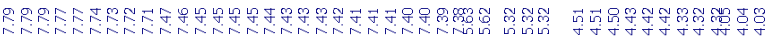

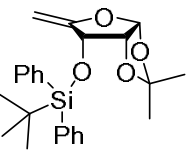

$4 e$

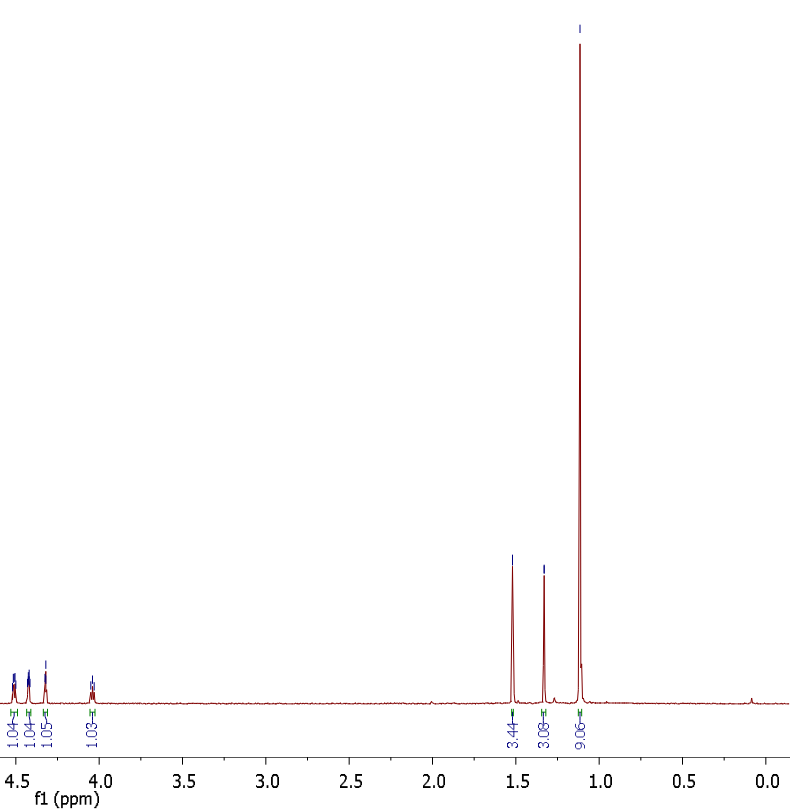

\section{${ }^{13} \mathrm{C}\left\{{ }^{\mathrm{H}} \mathrm{H}\right\} \mathrm{NMR}, 100 \mathrm{MHz}, \mathrm{CD}_{2} \mathrm{Cl}_{2}$}

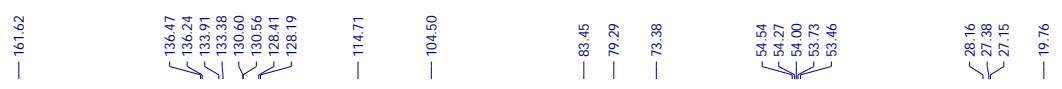
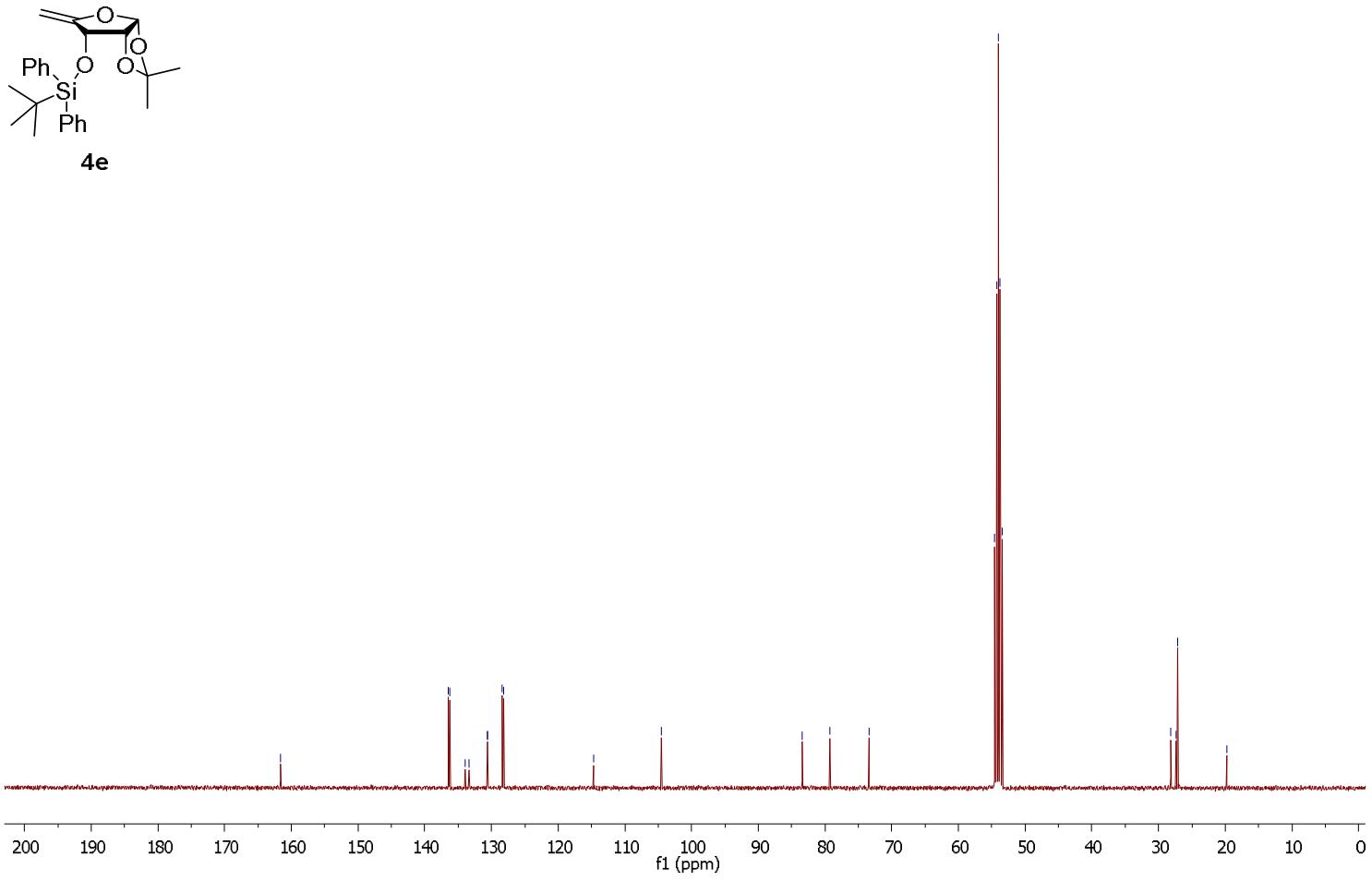

S-15 
${ }^{1} \mathrm{H}$ NMR, $400 \mathrm{MHz}, \mathrm{CDCl}_{3}$

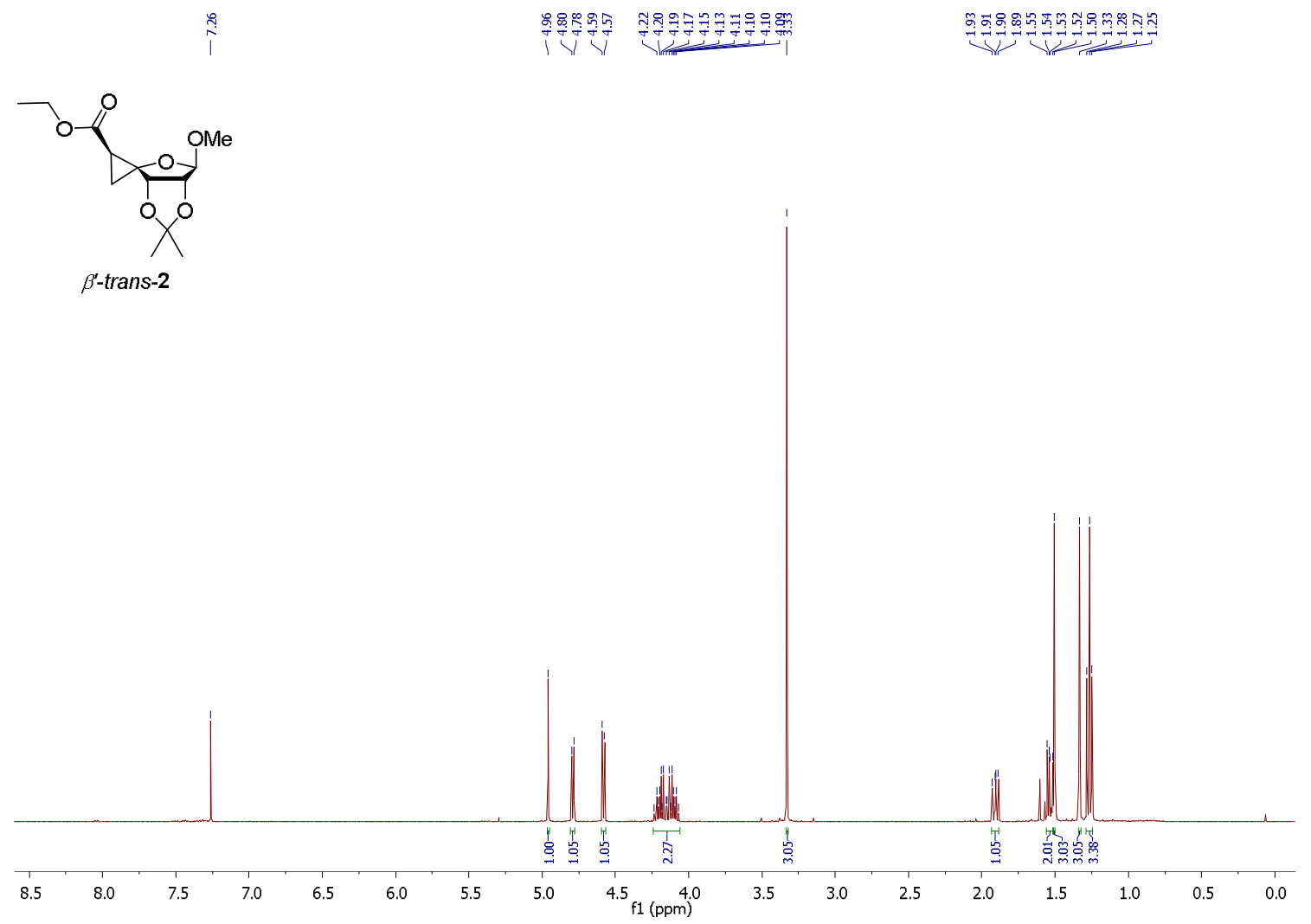

\section{${ }^{13} \mathrm{C}\left\{{ }^{\mathrm{H}} \mathrm{H}\right\} \mathrm{NMR}, 100 \mathrm{M} \mathrm{Hz}, \mathrm{CDCl}_{3}$}

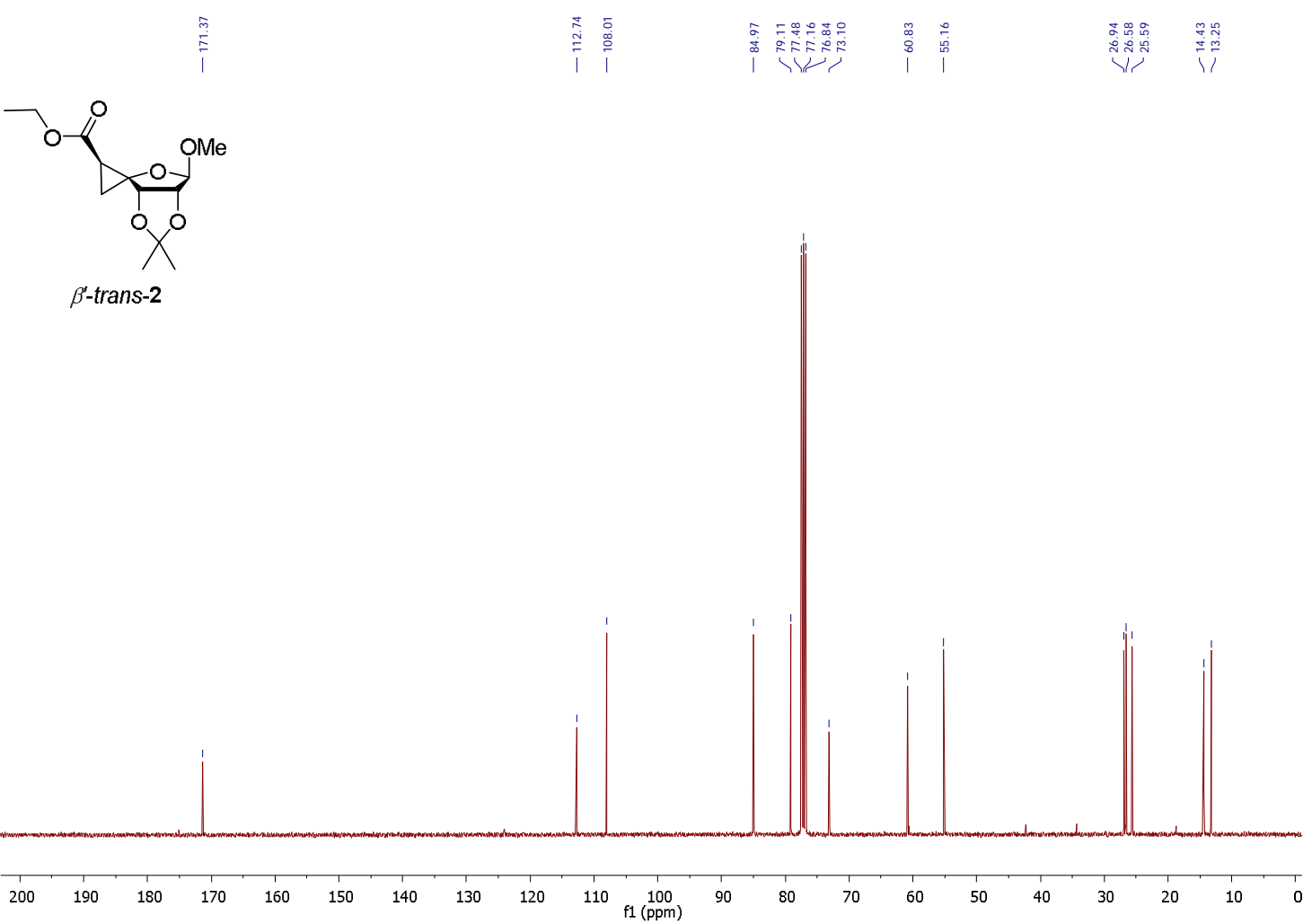

S-16 
${ }^{1} \mathrm{H}$ NMR, $400 \mathrm{MHz}, \mathrm{CDCl}_{3}$

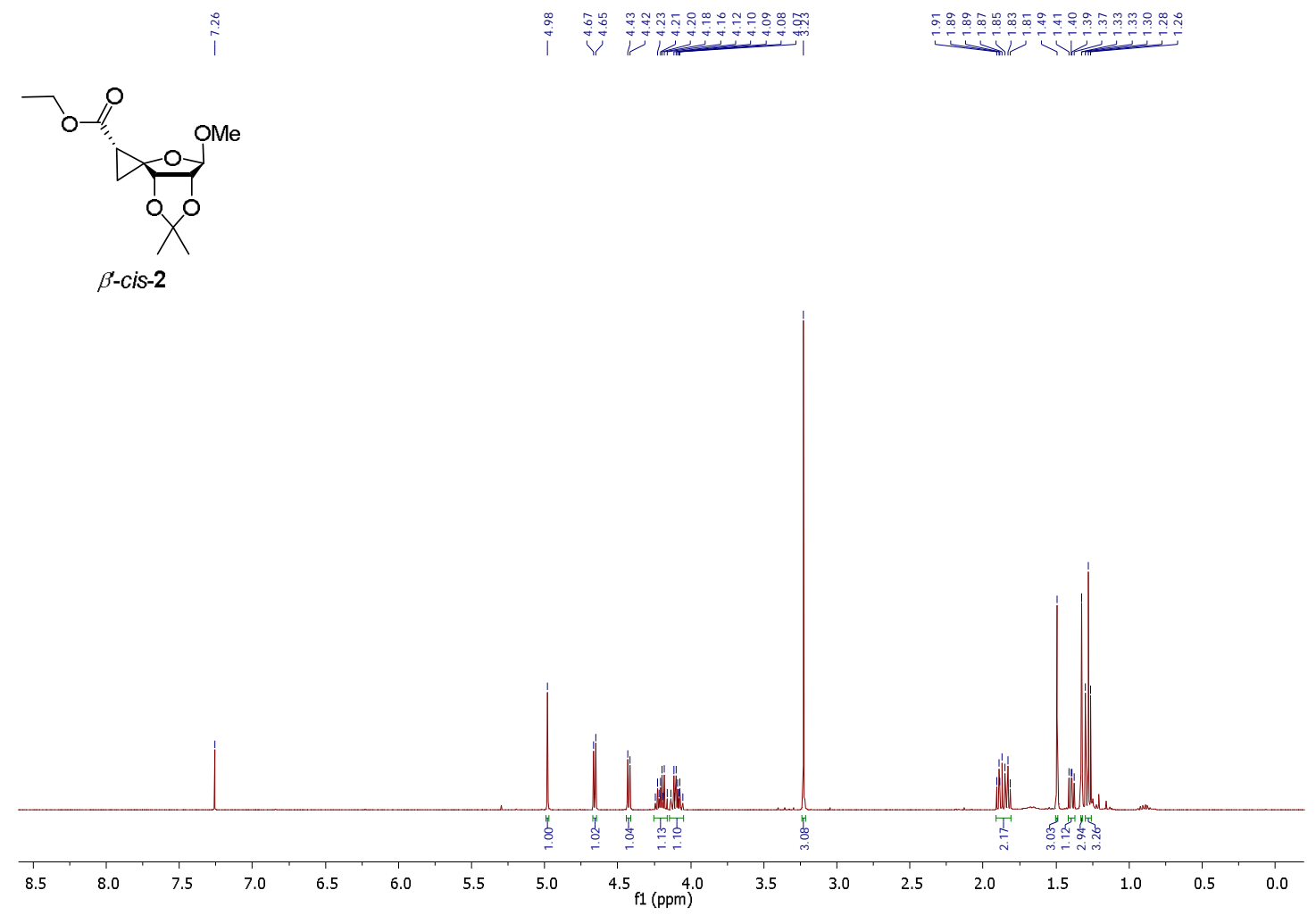

\section{${ }^{13} \mathrm{C}\left\{{ }^{1} \mathrm{H}\right\}$ NMR, $100 \mathrm{M} \mathrm{Hz}, \mathrm{CDCl}_{3}$}
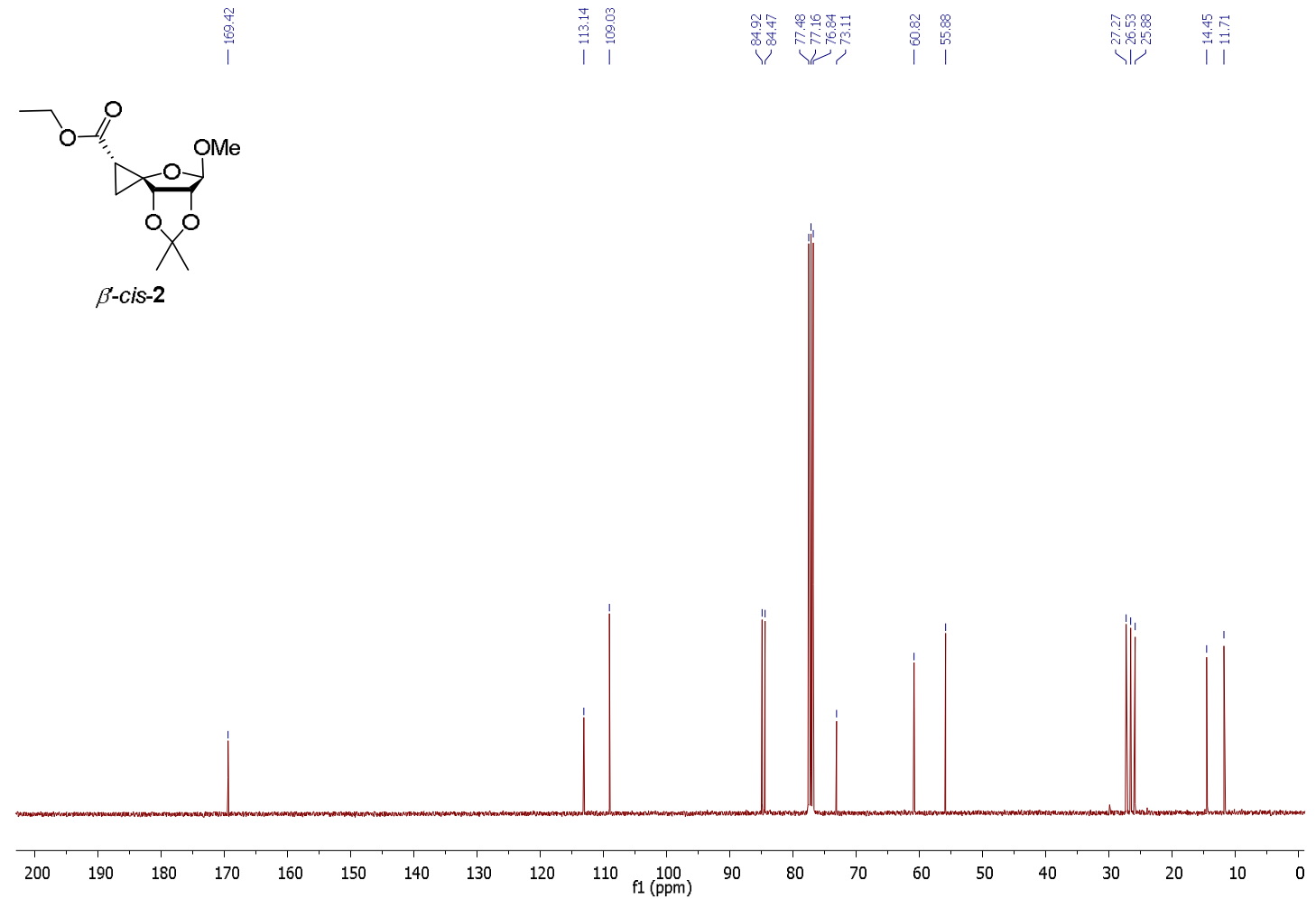
${ }^{1} \mathrm{H}$ NMR, $400 \mathrm{MHz}, \mathrm{CDCl}_{3}$

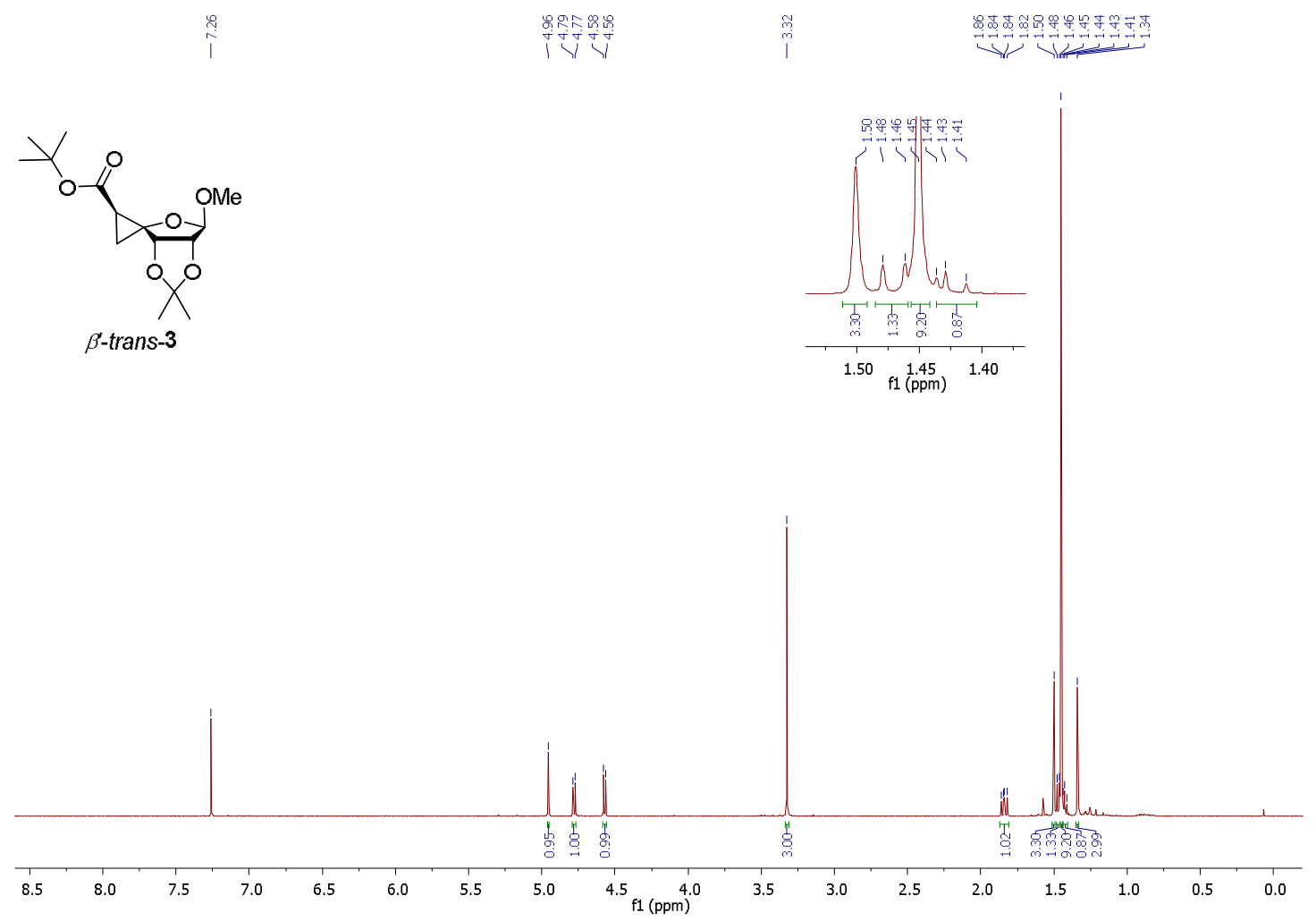

${ }^{13} \mathrm{C}\left\{{ }^{\mathrm{l}} \mathrm{H}\right\} \mathrm{NMR}, 100 \mathrm{M} \mathrm{Hz}, \mathrm{CDCl}_{3}$

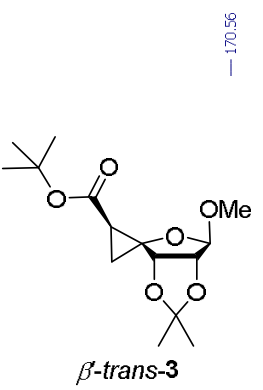

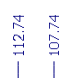

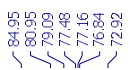

ل1

$\beta$-trans-3

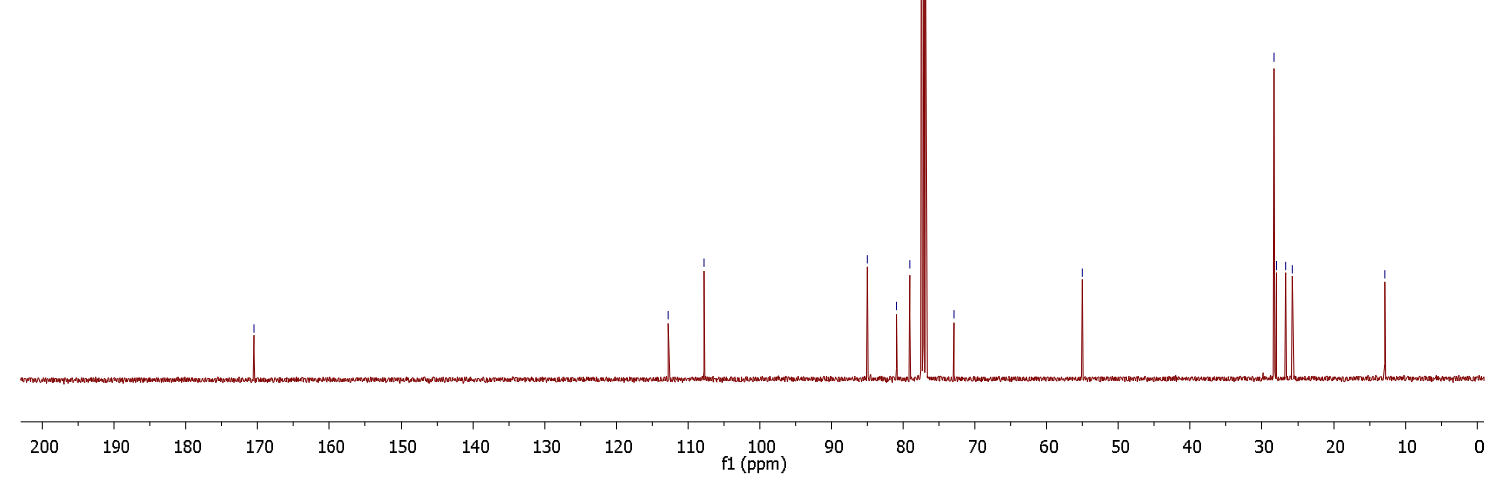

S-18 
${ }^{1} \mathrm{H} \mathrm{NMR}, 400 \mathrm{MHz}, \mathrm{CDCl}_{3}$

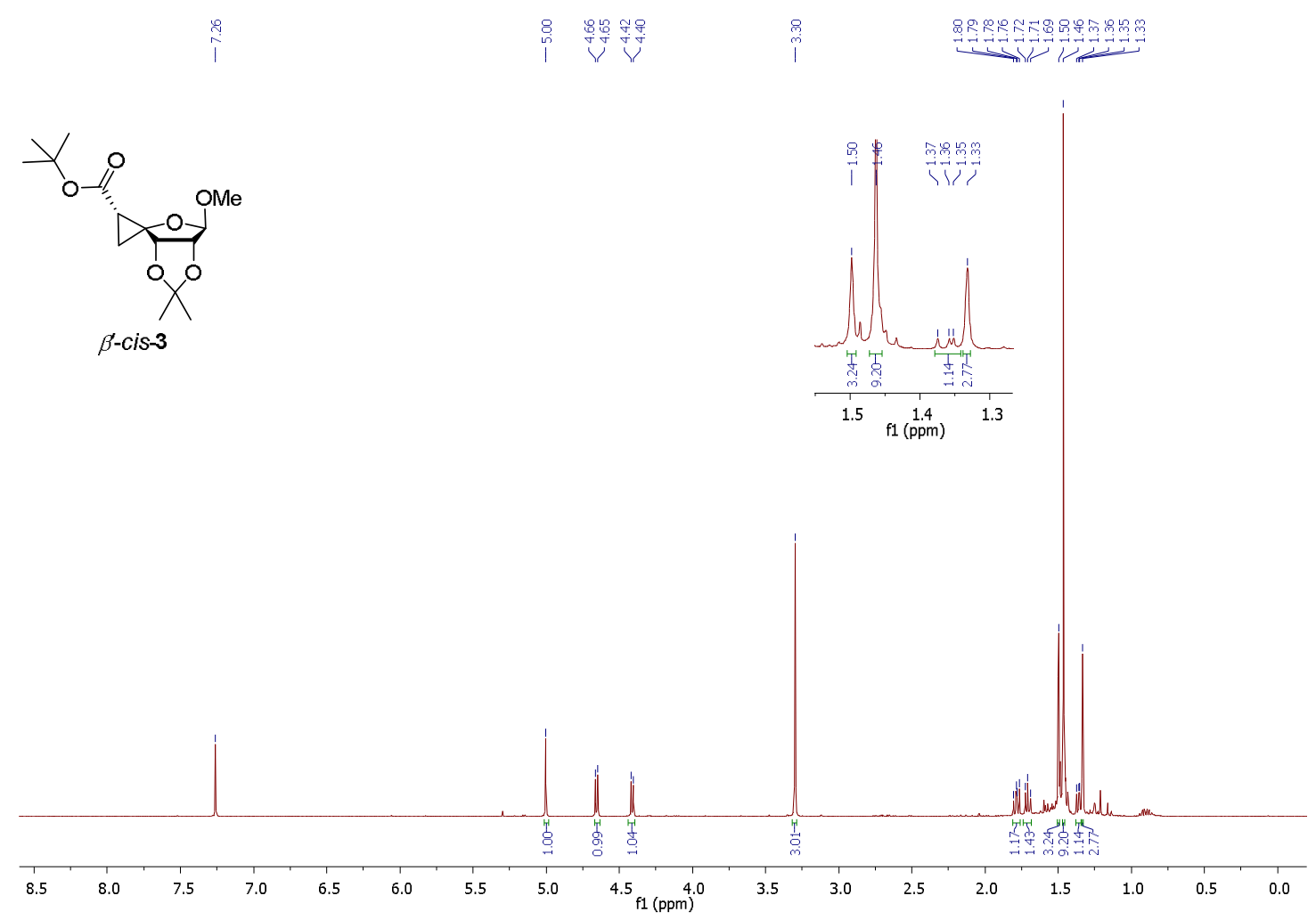

$\left.{ }^{13} \mathrm{C}^{1} \mathrm{H}\right\} \mathrm{NMR}, 100 \mathrm{M} \mathrm{Hz}, \mathrm{CDCl}_{3}$

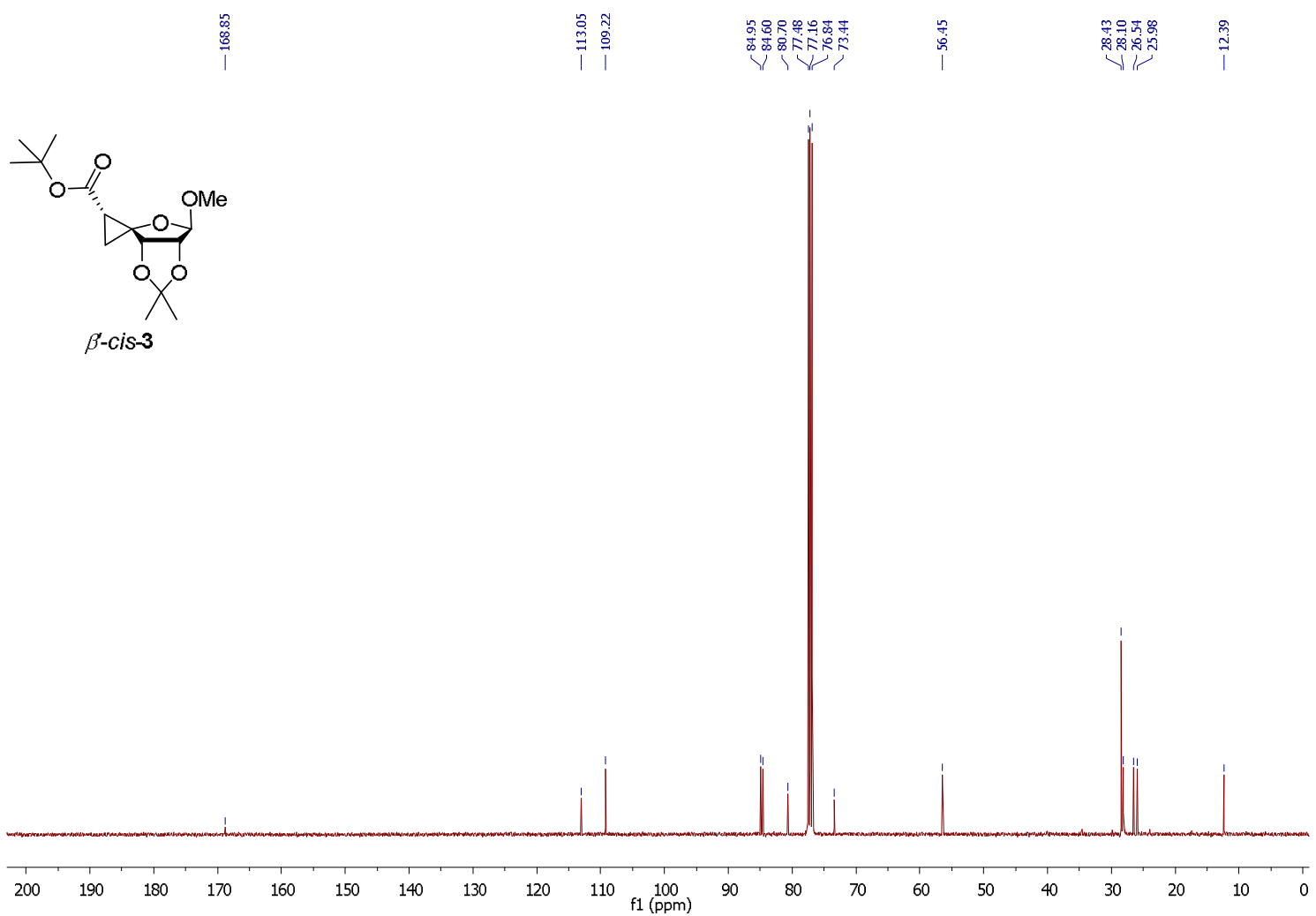

S-19 
${ }^{1} \mathrm{H}$ NMR, $400 \mathrm{MHz}, \mathrm{CDCl}_{3}$

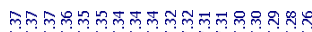

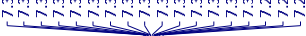

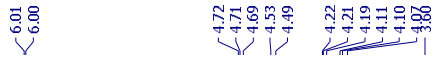

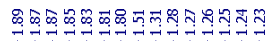
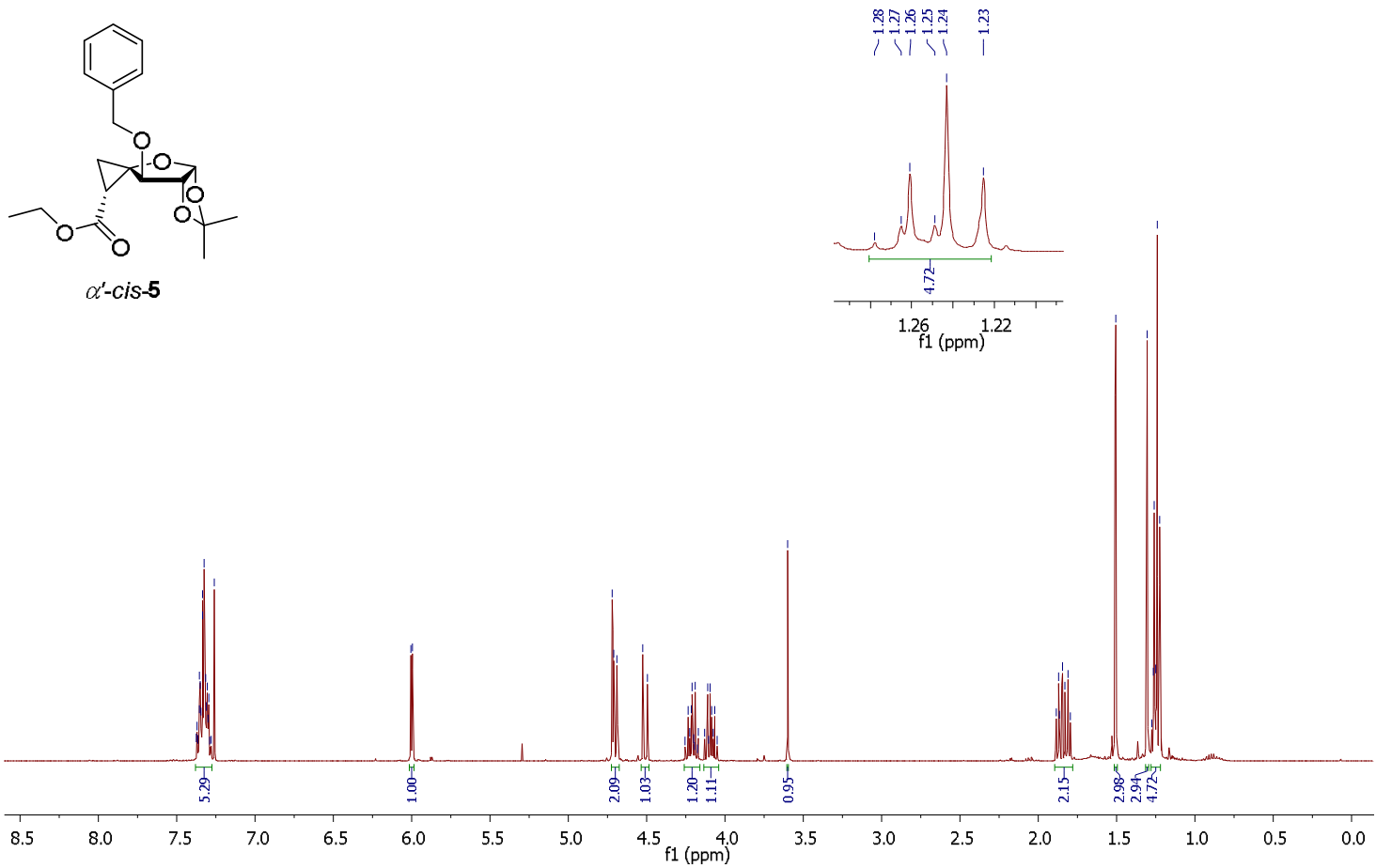

\section{${ }^{13} \mathrm{C}\{\mathrm{H} H\}$ NMR, $100 \mathrm{MHz}, \mathrm{CDCl}_{3}$}
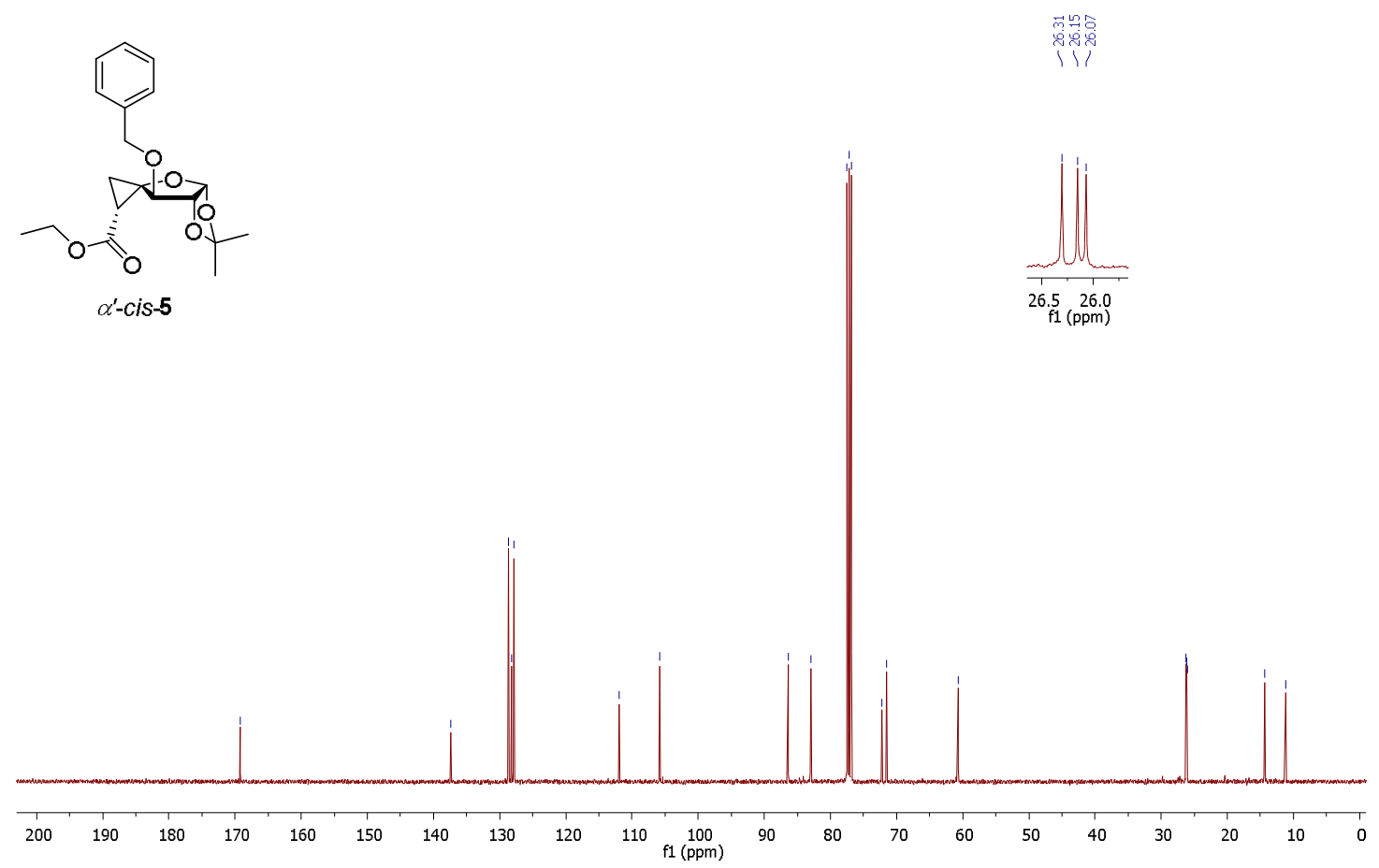
${ }^{1} \mathrm{H}$ NMR, $400 \mathrm{MHz}, \mathrm{CDCl}_{3}$ (contains $34 \%$ of diethyl maleate [*])

Vั

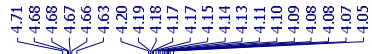

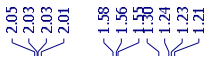
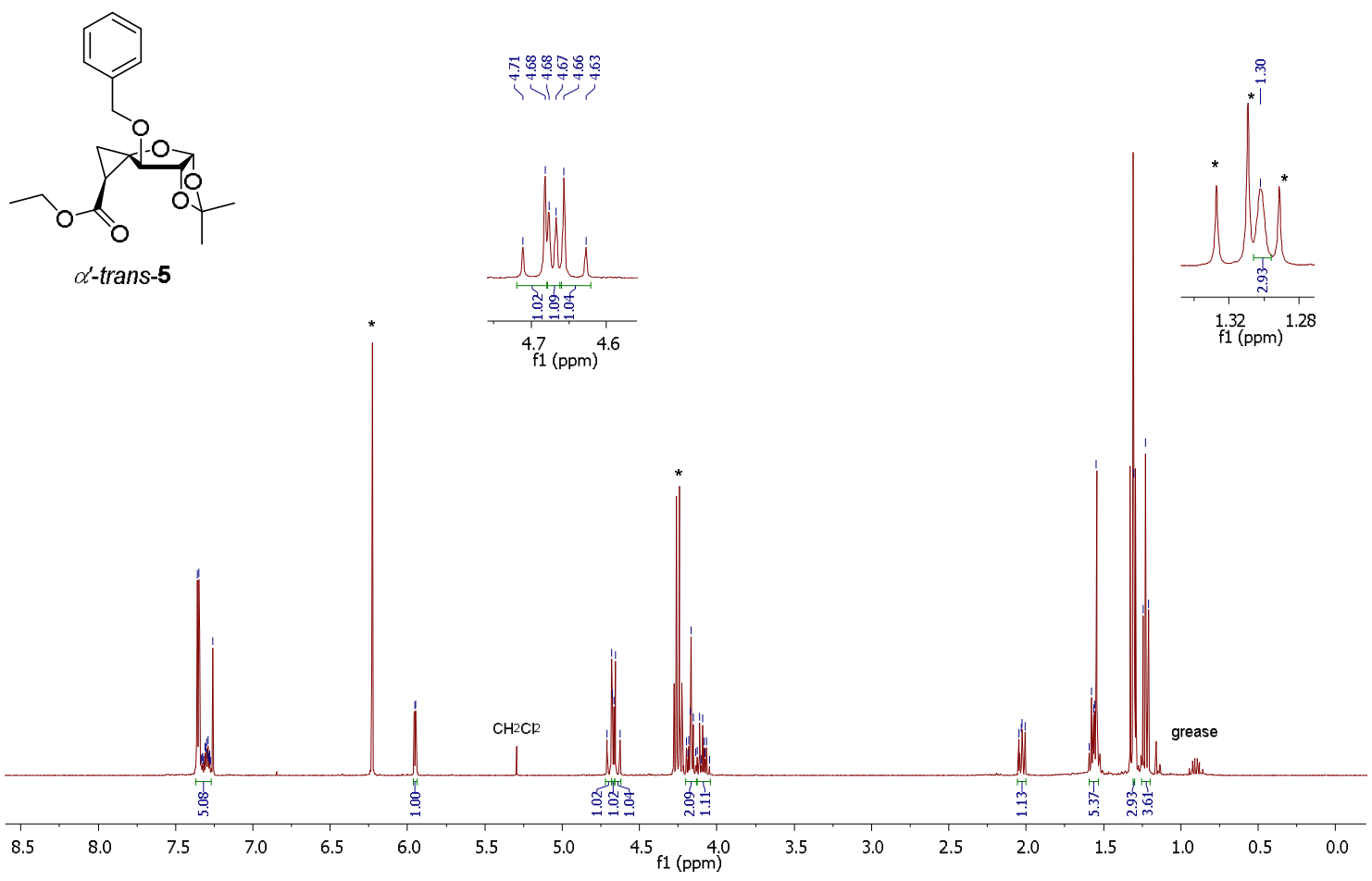

${ }^{13} \mathrm{C}\left\{{ }^{1} \mathrm{H}\right\} \mathrm{NMR}, 100 \mathrm{M} \mathrm{Hz}, \mathrm{CDCl}_{3}$ (contains $34 \%$ of diethyl maleate $\left[{ }^{*}\right]$ )

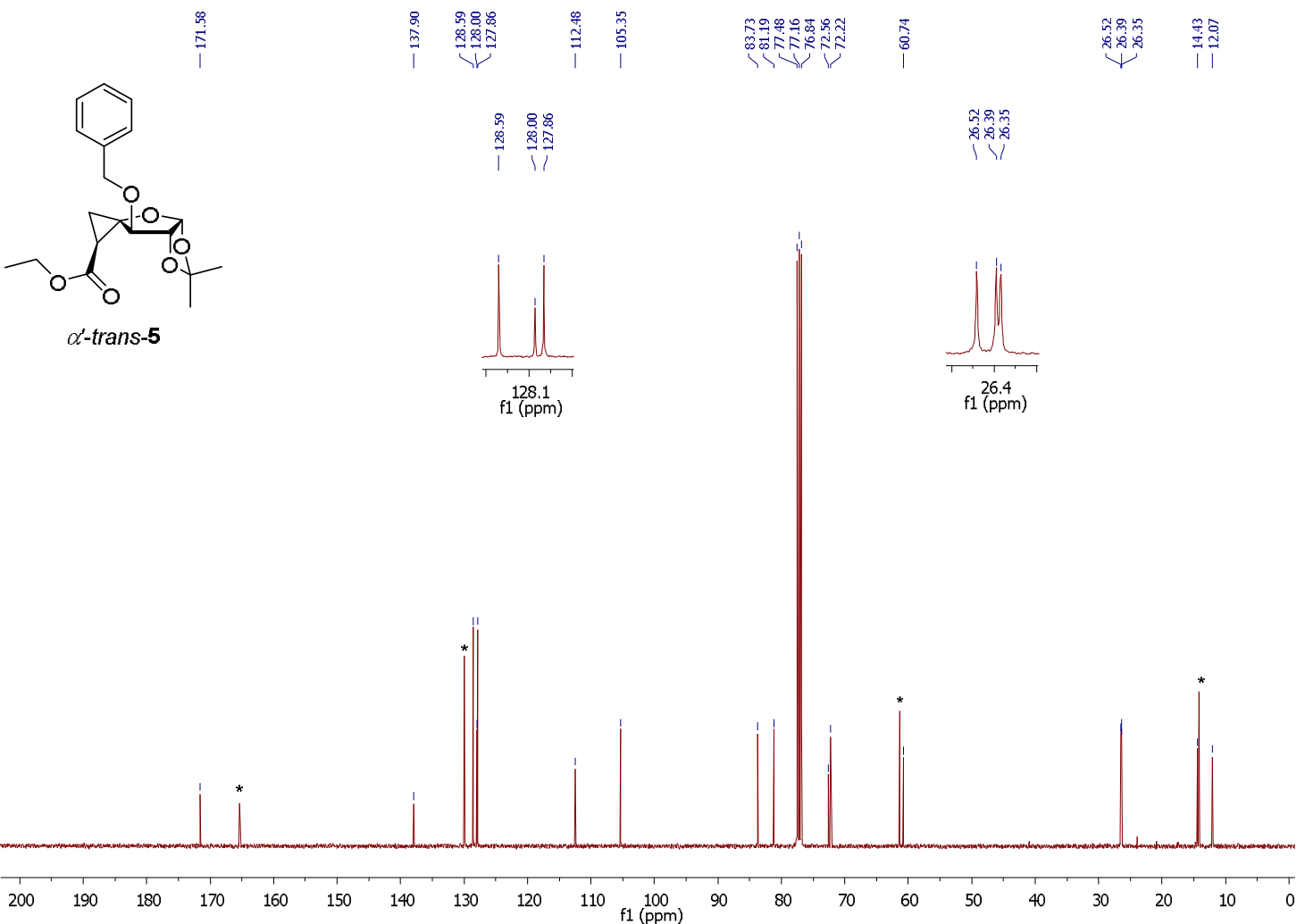


${ }^{1} \mathrm{H} N \mathrm{NM}, 400 \mathrm{MHz}, \mathrm{CDCl}_{3}$

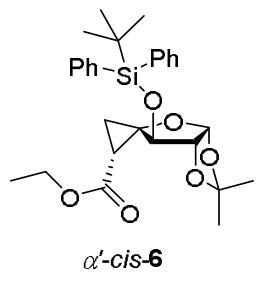

${ }^{13} \mathrm{C}\{\mathrm{H}\} \mathrm{NMR}, 100 \mathrm{MHz}, \mathrm{CDCl}_{3}$
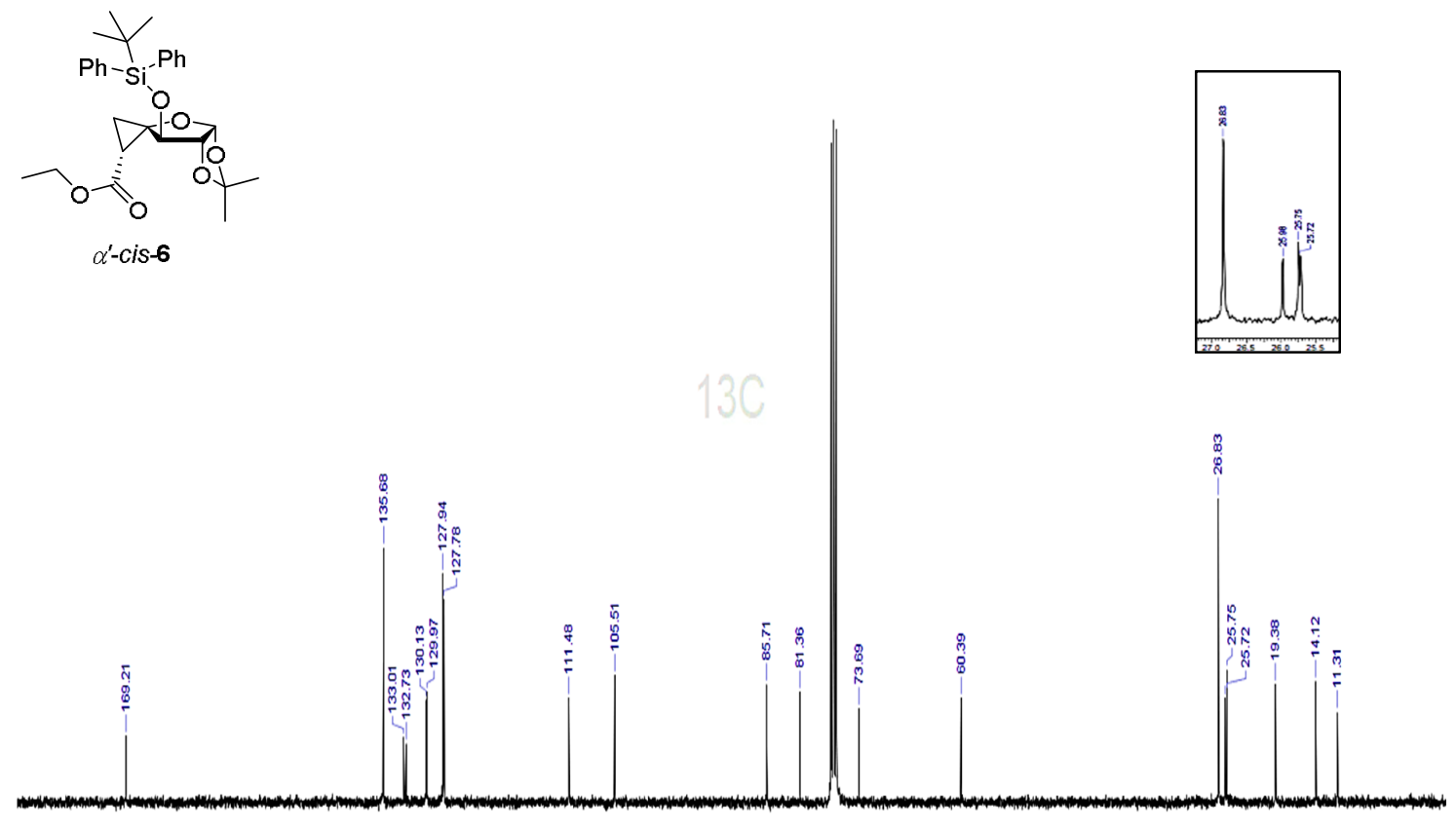

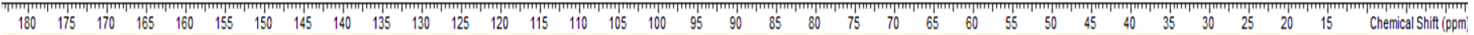




\section{${ }^{1} \mathrm{H}$ NMR, $400 \mathrm{MHz}, \mathrm{CDCl}_{3}$}

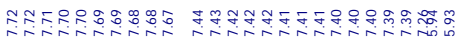

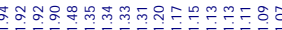

${\mathrm{Ph}-\mathrm{Si}^{-} \mathrm{Ph}}_{-\mathrm{O}}$

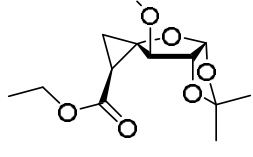

$\alpha^{\prime}$-trans-6

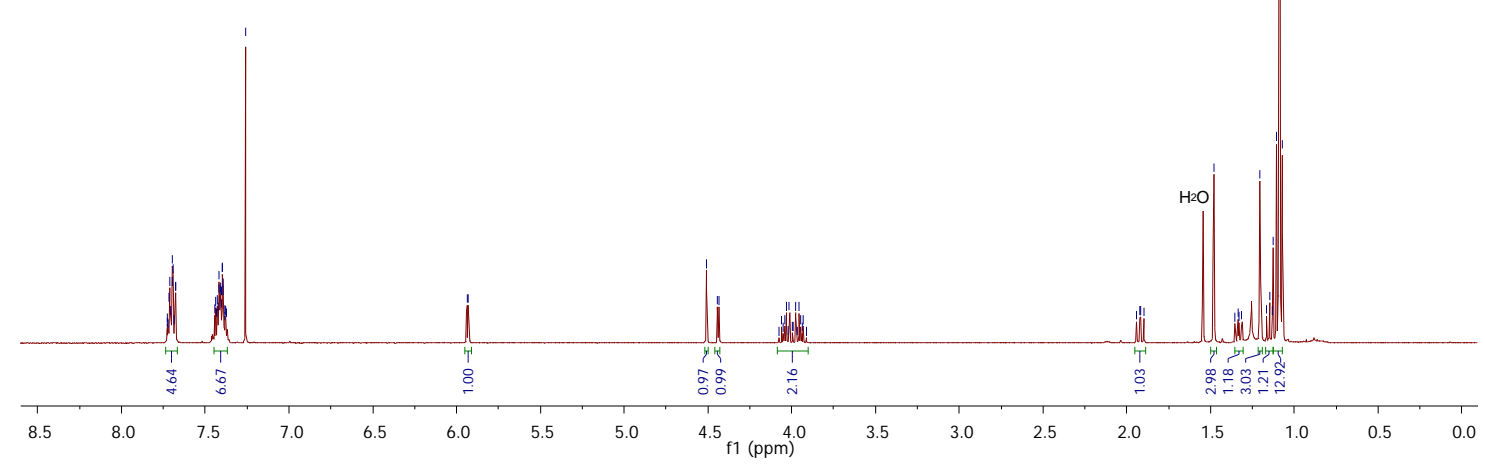

${ }^{13} \mathrm{C}\left\{{ }^{1} \mathrm{H}\right\} \mathrm{NMR}, 100 \mathrm{M} \mathrm{Hz}, \mathrm{CDCl}_{3}$

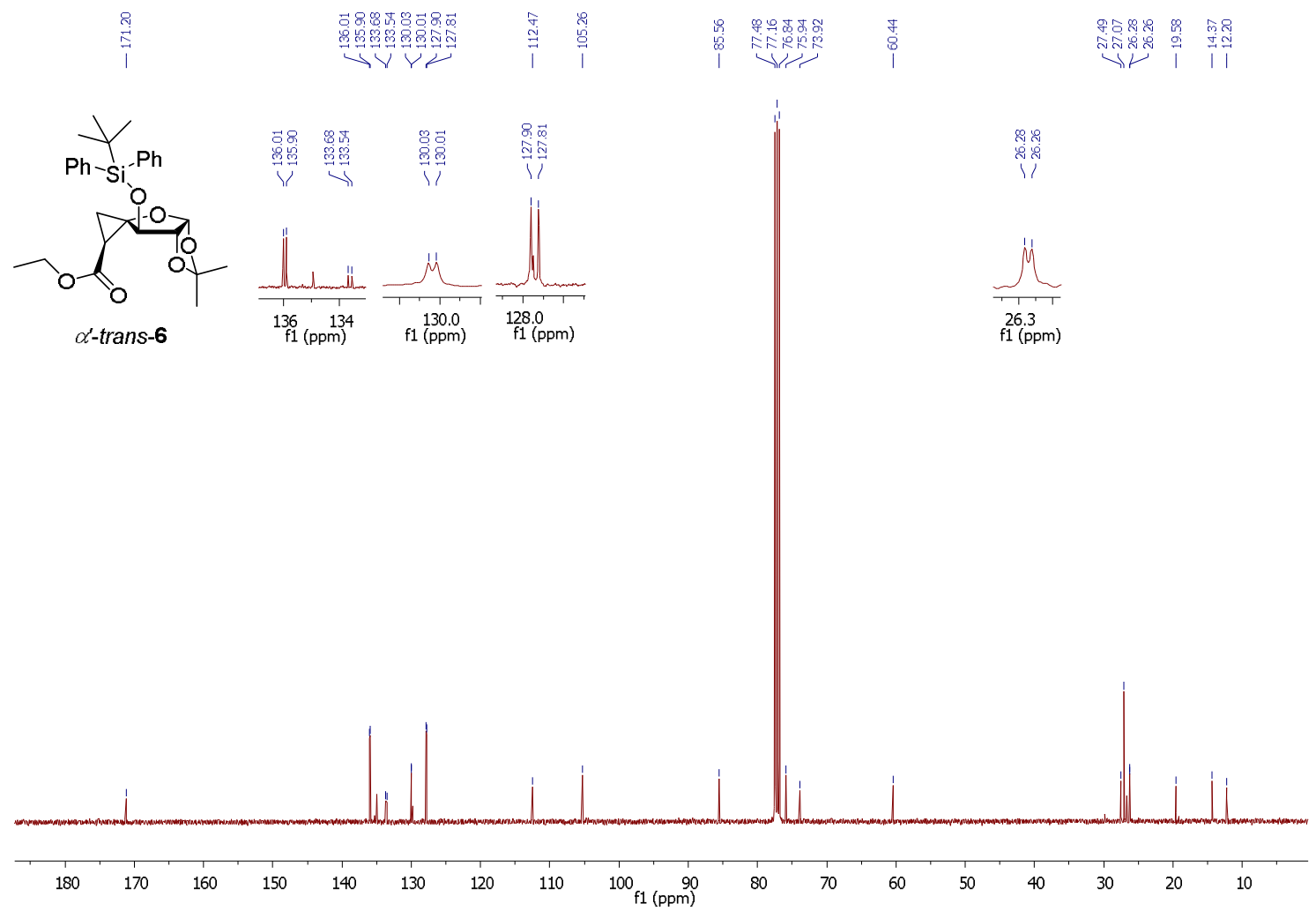


${ }^{1} \mathrm{H}$ NMR, $400 \mathrm{MHz}, \mathrm{CDCl}_{3}$

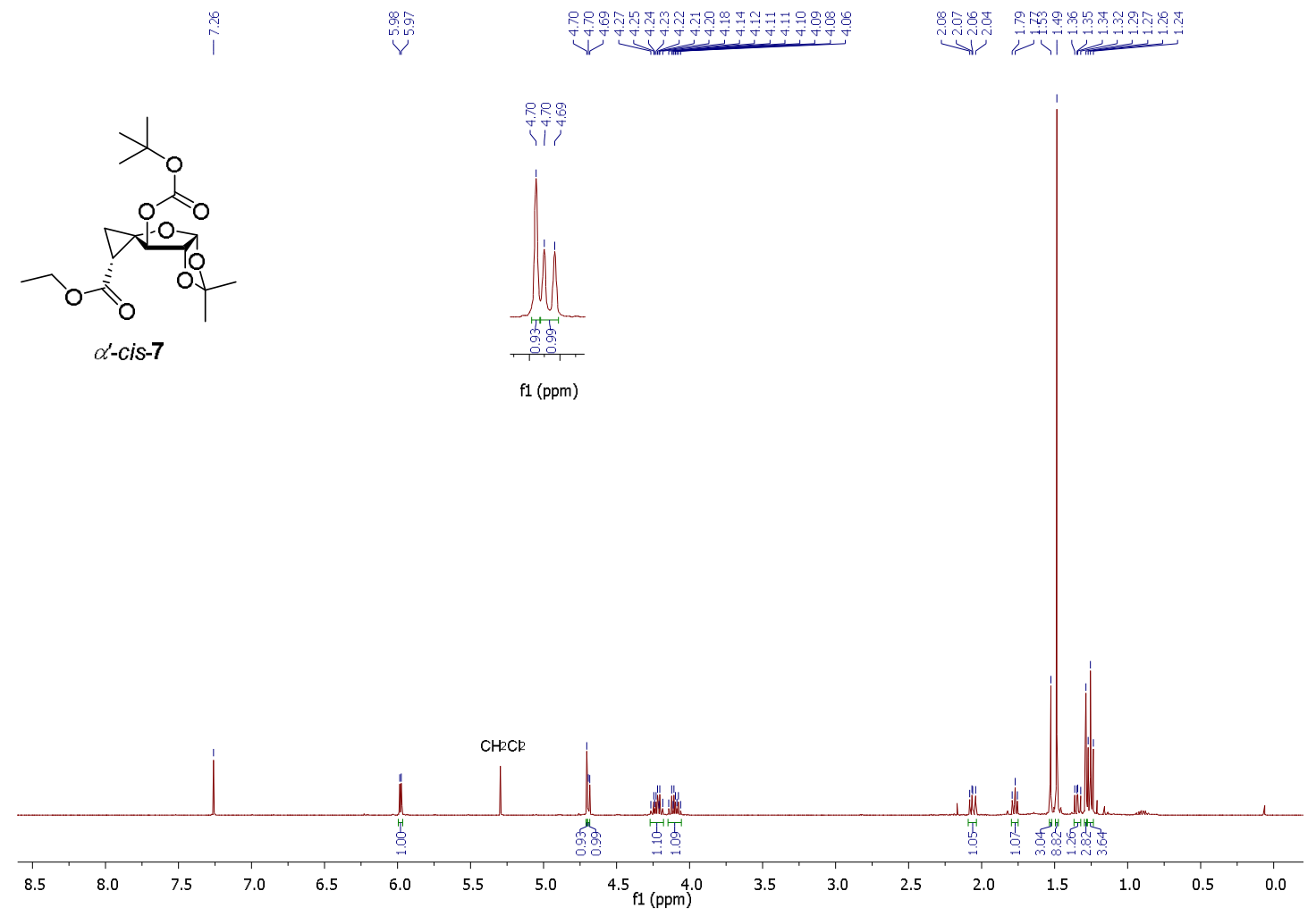

\section{${ }^{13} \mathrm{C}\left\{{ }^{1} \mathrm{H}\right\} \mathrm{NMR}, 100 \mathrm{M} \mathrm{Hz}, \mathrm{CDCl}_{3}$}

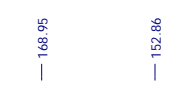

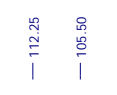

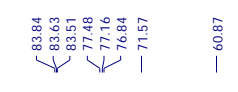

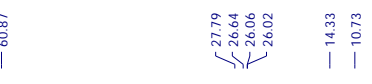
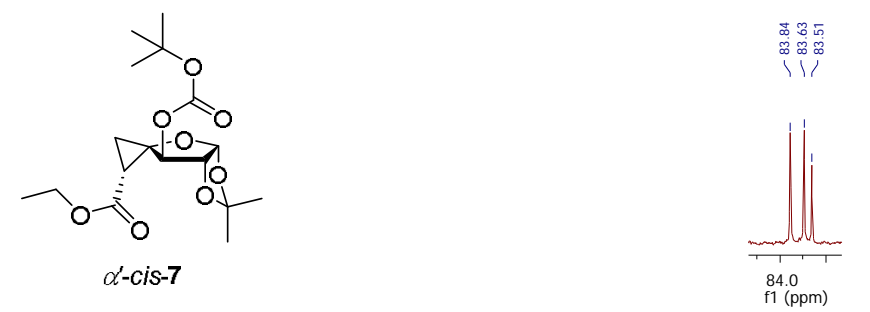

I
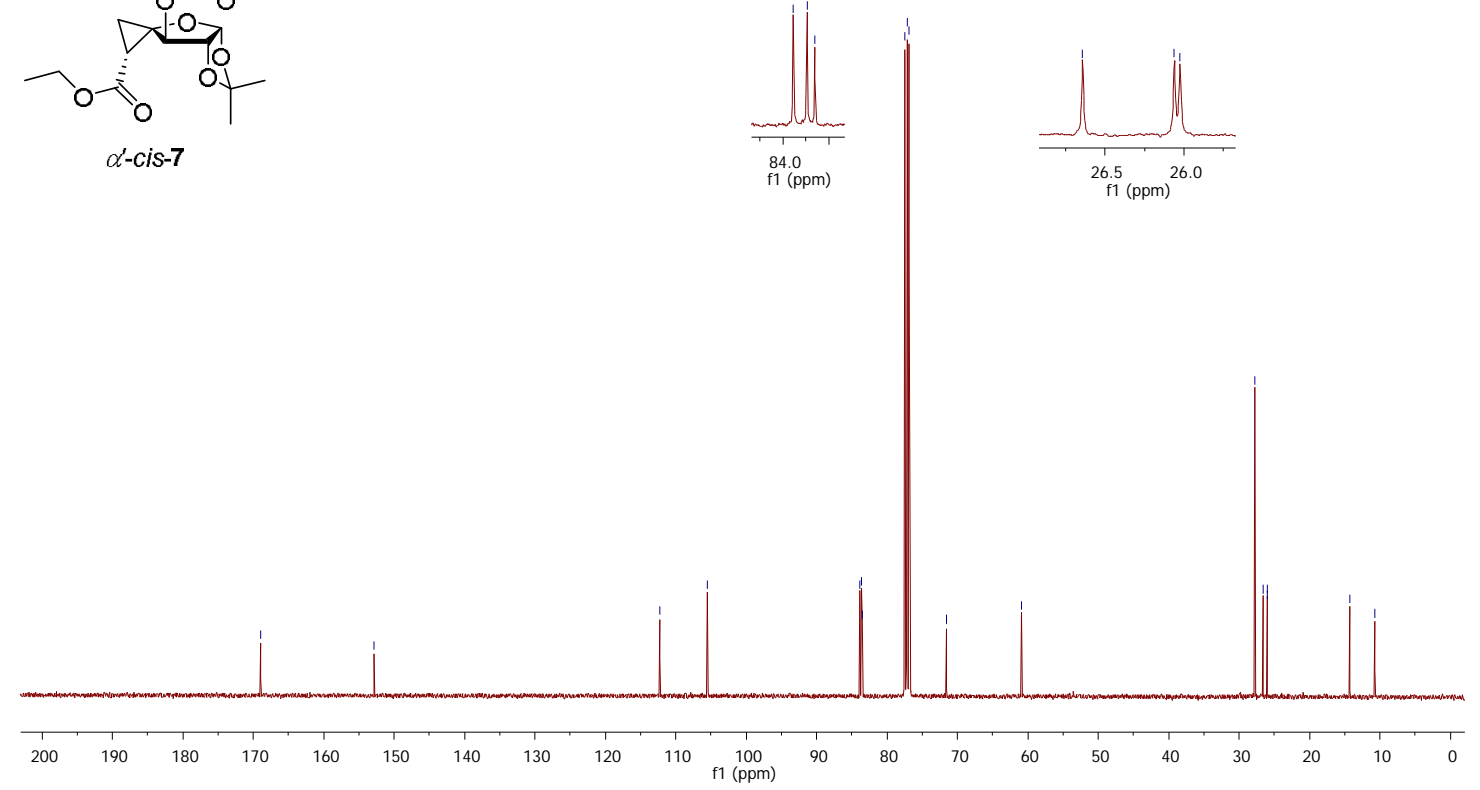

S-24 
${ }^{1} \mathrm{H}$ NMR, $400 \mathrm{MHz}, \mathrm{CDCl}_{3}$

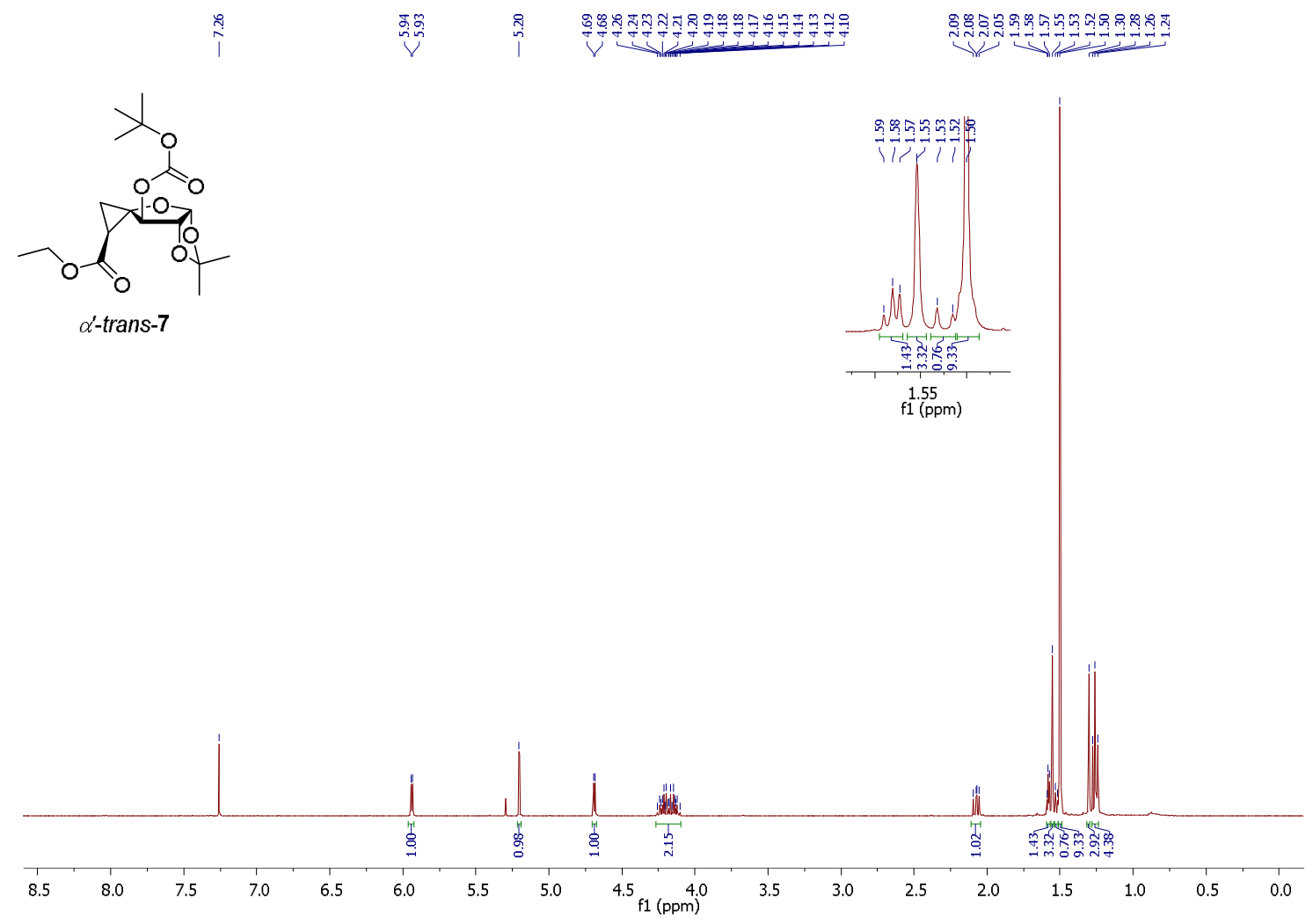

$\left.{ }^{13} \mathrm{C}^{\mathrm{l}} \mathrm{H}\right\} \mathrm{NMR}, 100 \mathrm{MHz}, \mathrm{CDCl}_{3}$

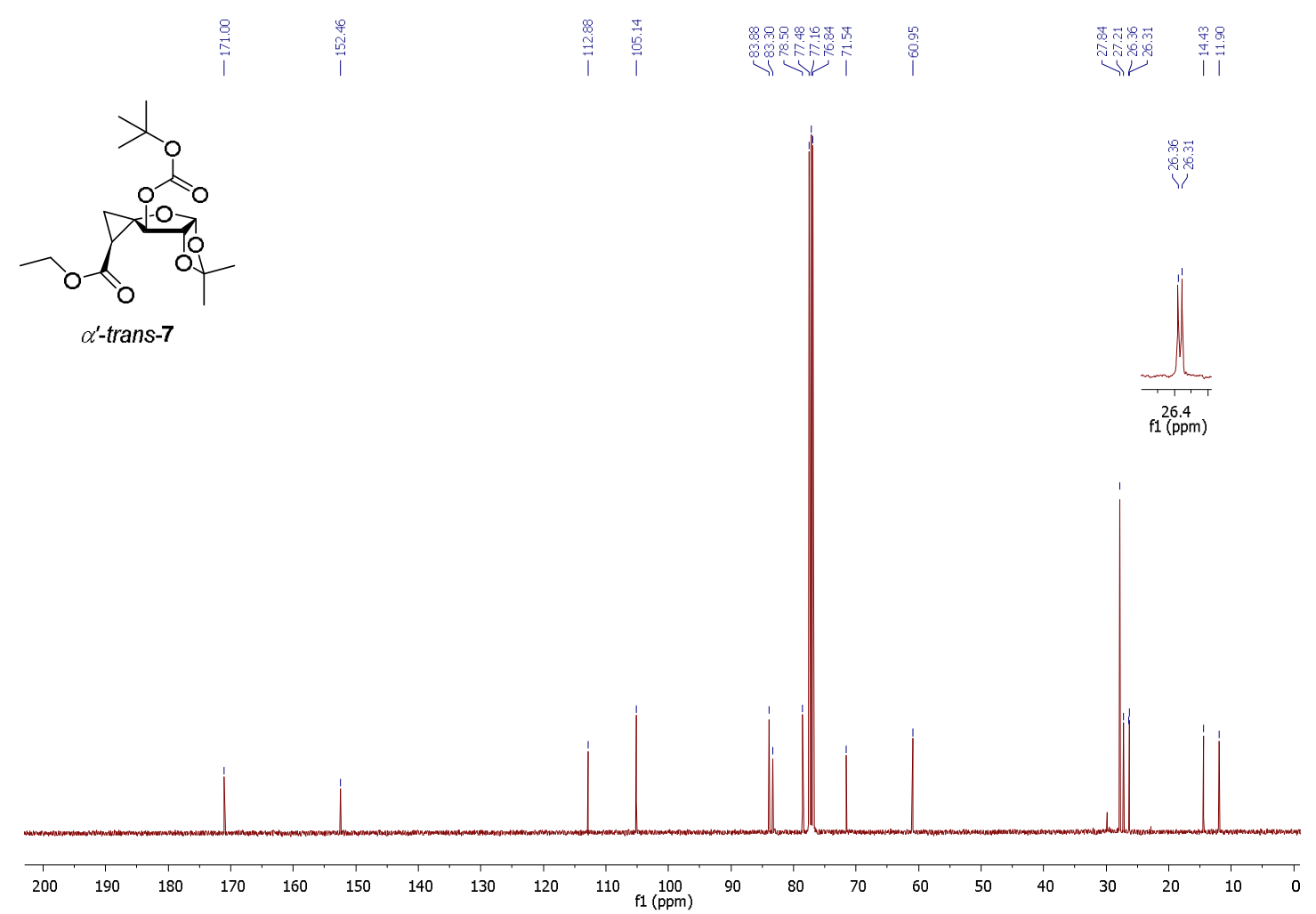

S-25 
${ }^{1} \mathrm{H}$ NM R, $400 \mathrm{MHz}, \mathrm{CD}_{2} \mathrm{Cl}_{2}$ (contains traces of diethyl maleate [*])

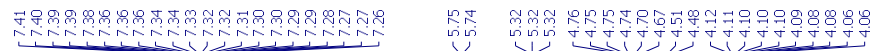

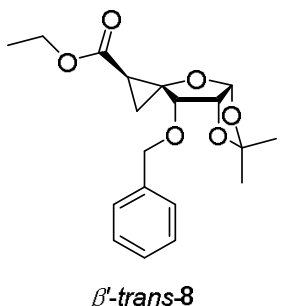

$\beta^{\prime}$-trans-8
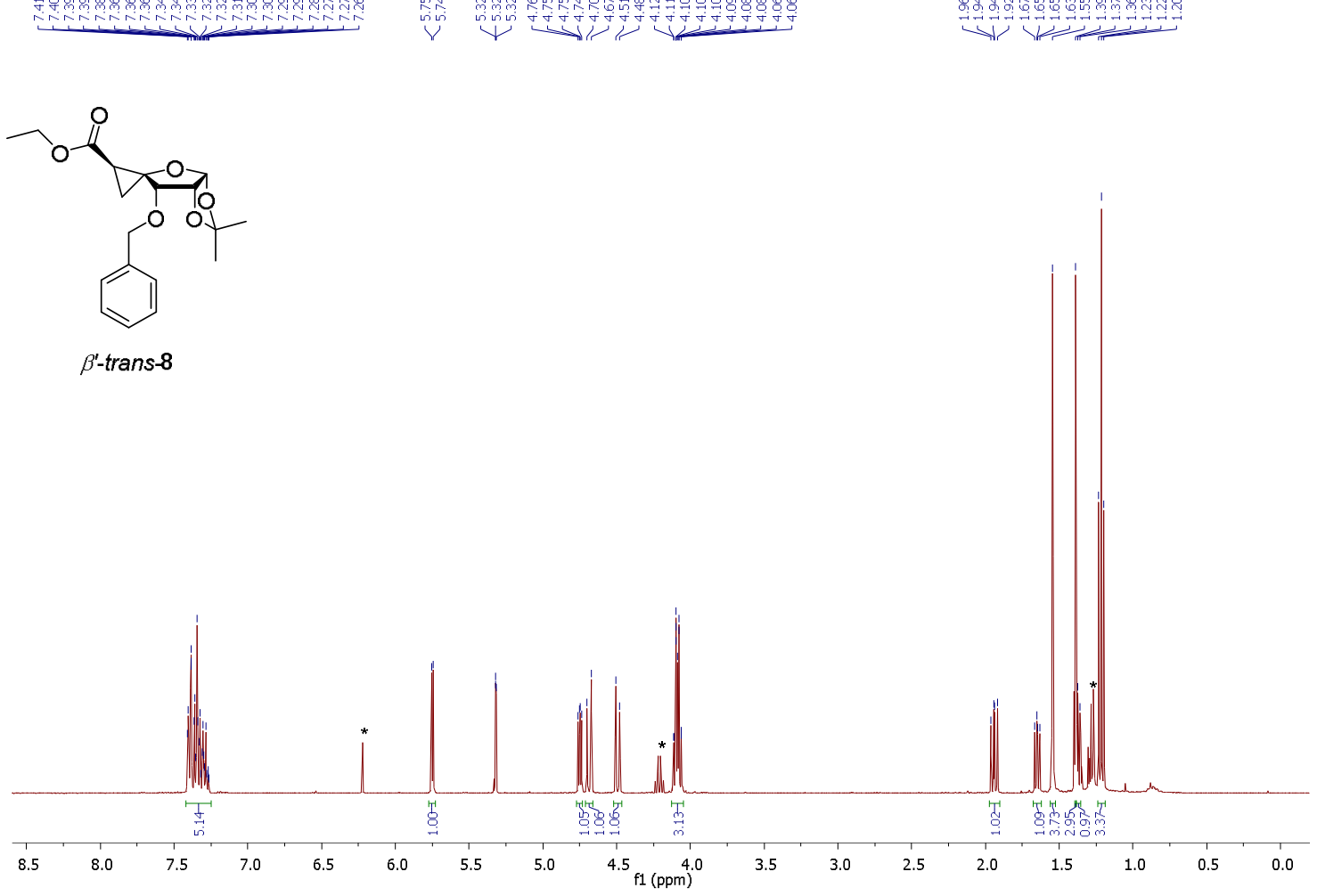

${ }^{13} \mathrm{C}\left\{{ }^{\mathrm{H}} \mathrm{H}\right\} \mathrm{NMR}, 126 \mathrm{MHz}, \mathrm{CD}_{2} \mathrm{Cl}_{2}$
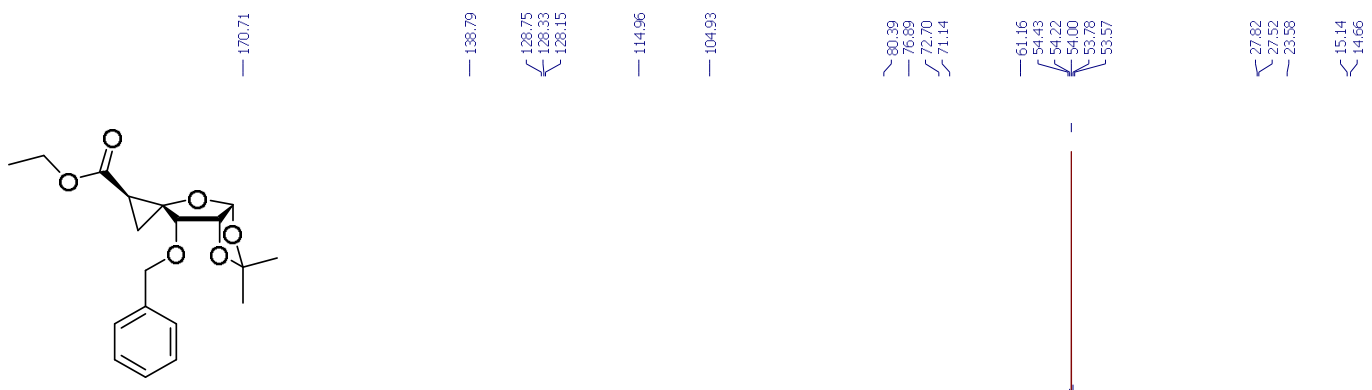

$\beta^{\prime}$-trans-8

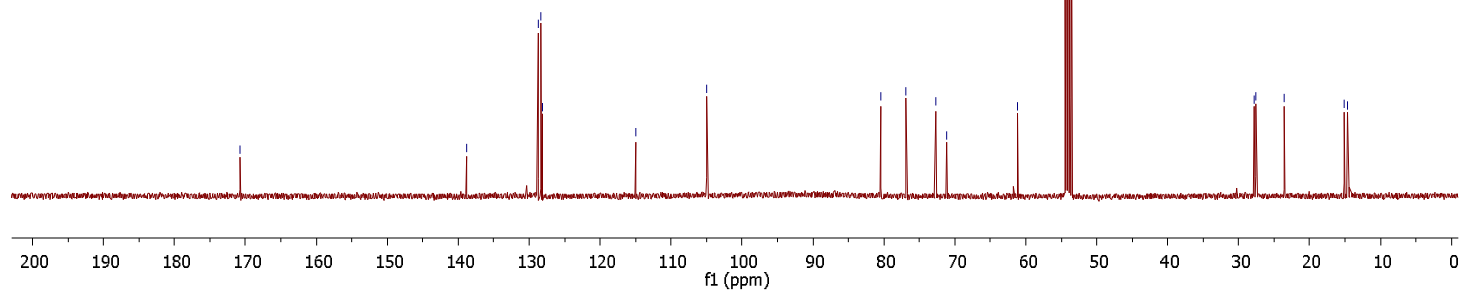


${ }^{1} \mathrm{H}$ NMR, $400 \mathrm{MHz}, \mathrm{CD}_{2} \mathrm{Cl}_{2}$

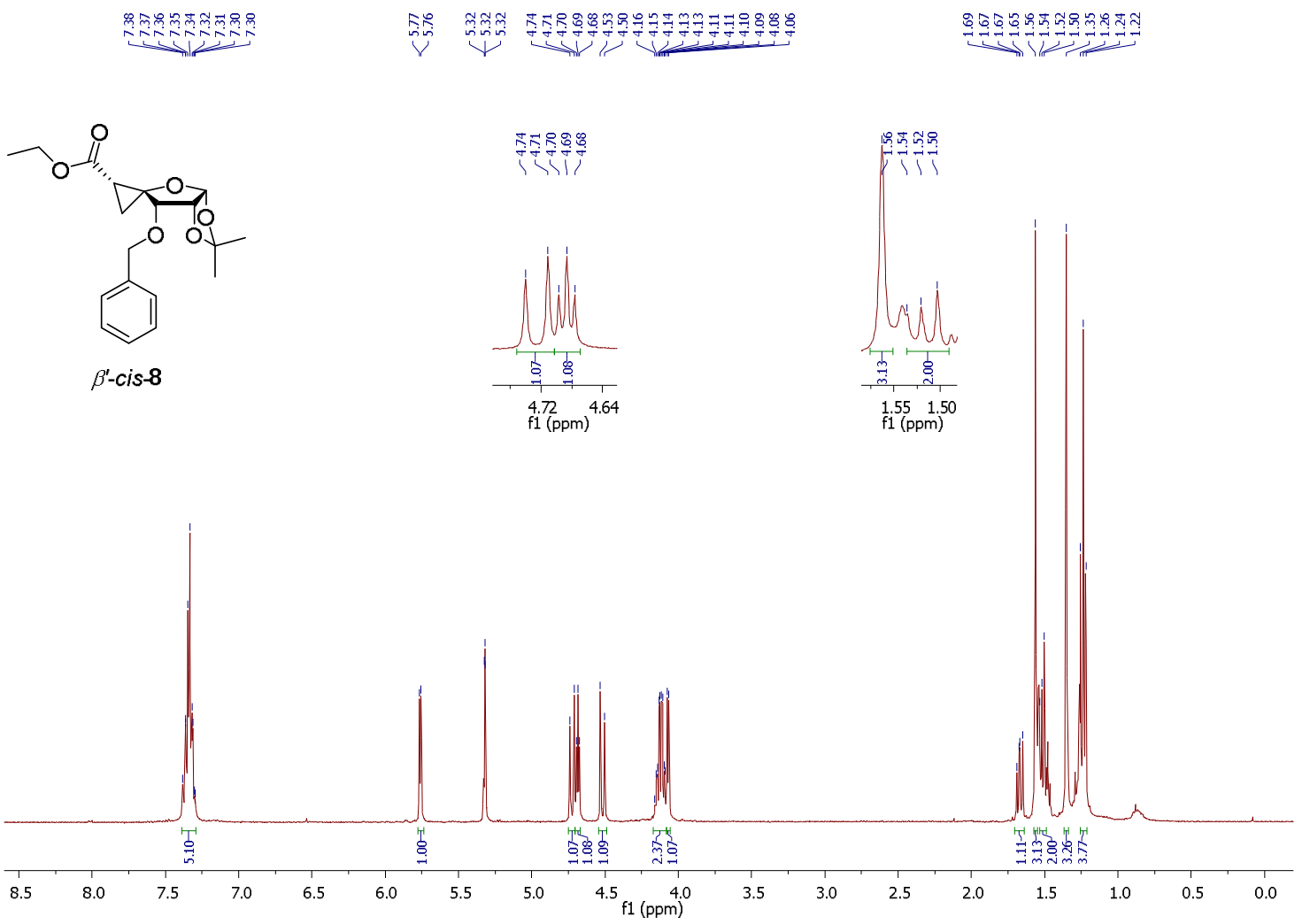

${ }^{13} \mathrm{C}\left\{{ }^{\mathrm{l}} \mathrm{H}\right\} \mathrm{NMR}, 100 \mathrm{MHz}, \mathrm{CD}_{2} \mathrm{Cl}_{2}$
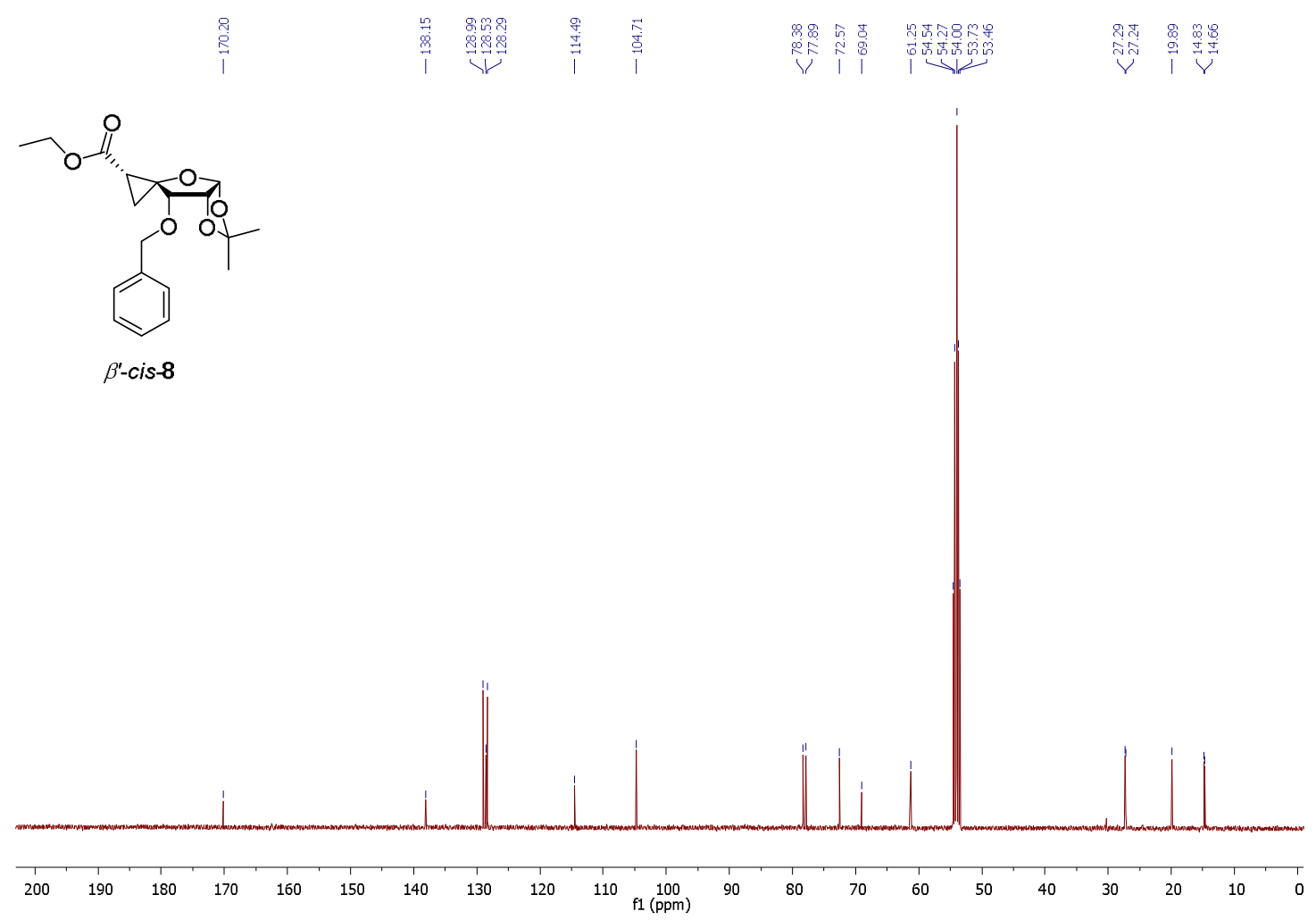
${ }^{1} \mathrm{H}$ NMR, $400 \mathrm{MHz}, \mathrm{CD}_{2} \mathrm{Cl}_{2}$

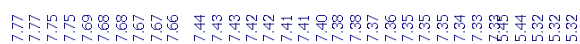

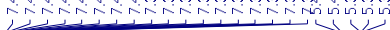

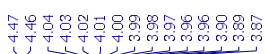

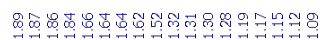

ㄴ.-1,

This of

$\beta$-trans-9

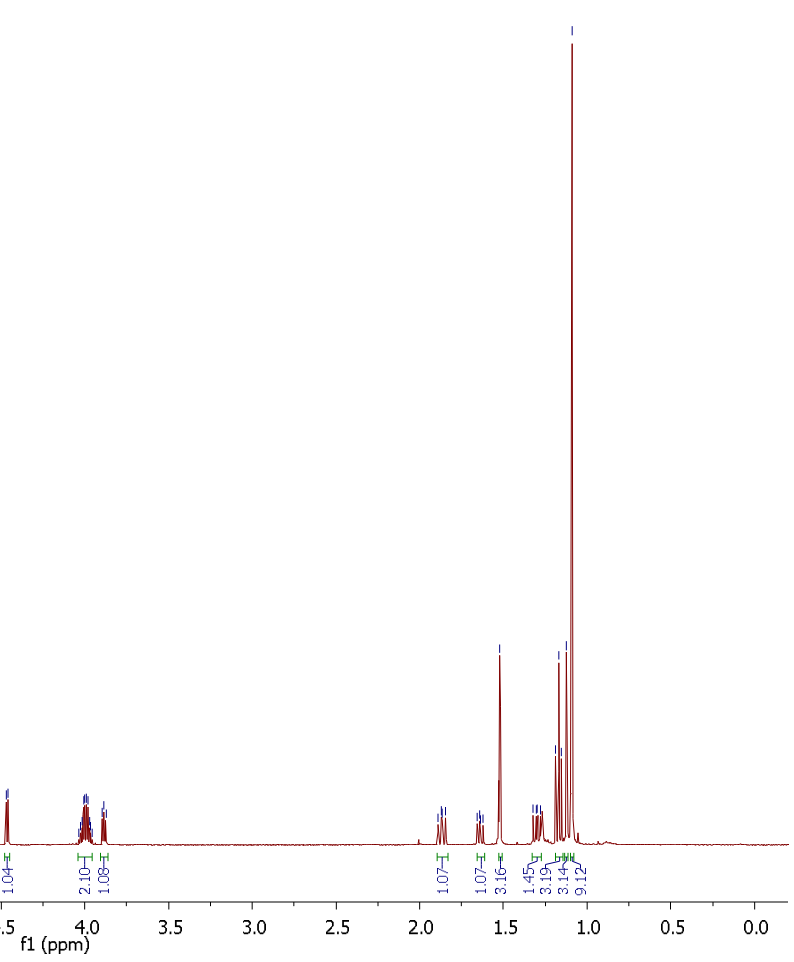

${ }^{13} \mathrm{C}\left\{{ }^{\mathrm{H}} \mathrm{H}\right\} \mathrm{NMR}, 75 \mathrm{MHz}, \mathrm{CD}_{2} \mathrm{Cl}_{2}$
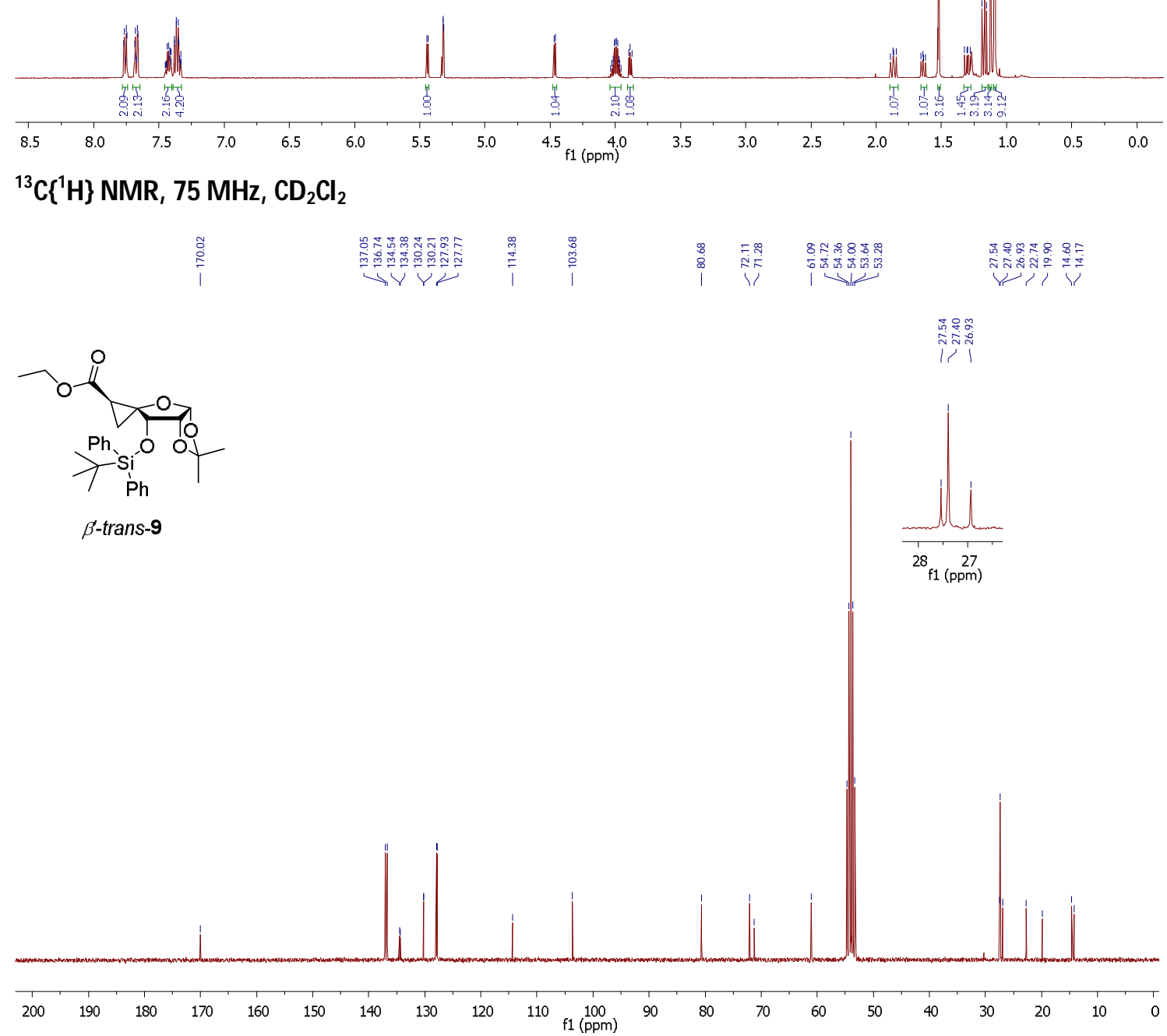

S-28 


\section{${ }^{1} \mathrm{H}$ NM R, $400 \mathrm{MHz}, \mathrm{CD}_{2} \mathrm{Cl}_{2}$ (contains traces of diethyl maleate [*])}
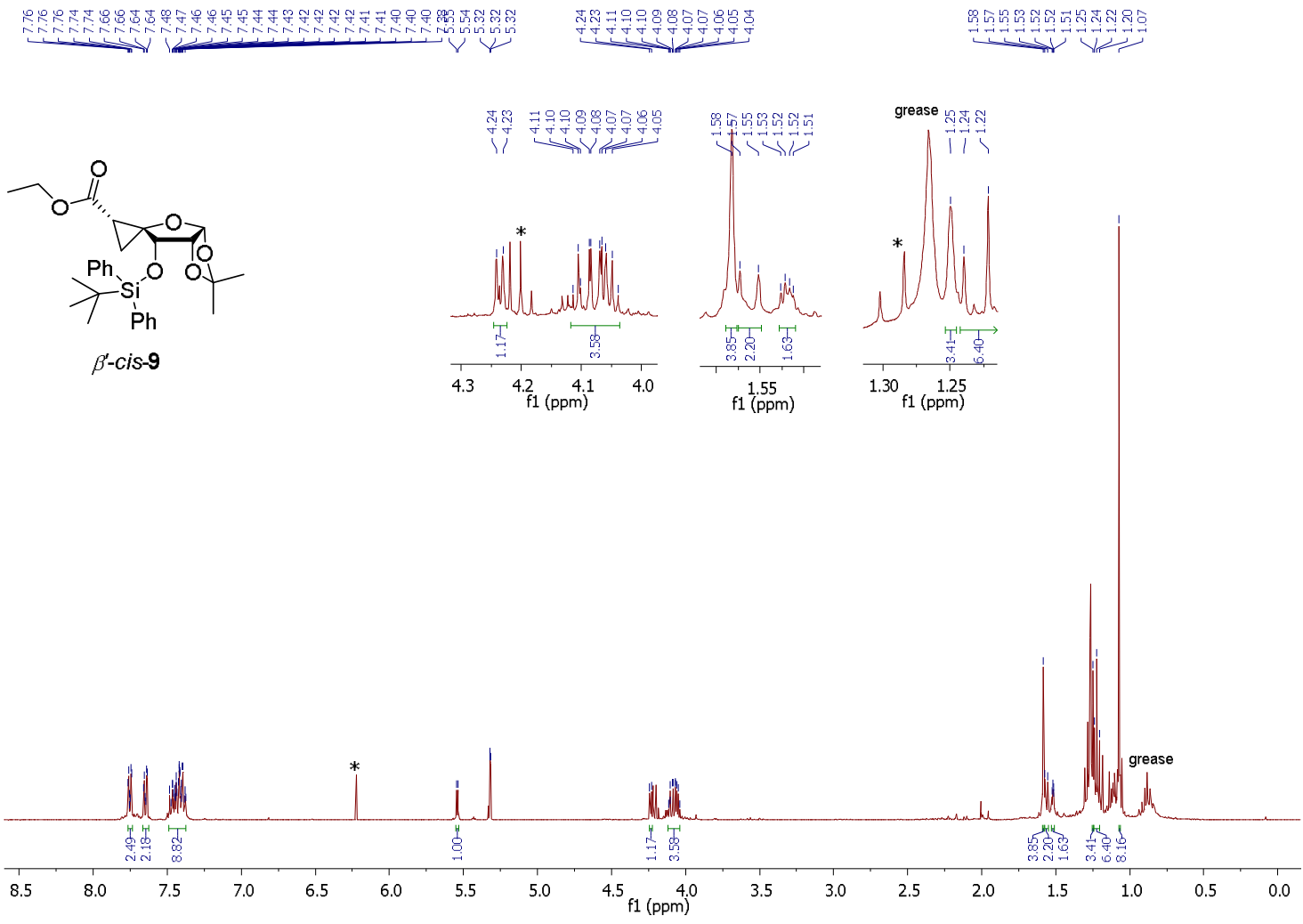

\section{${ }^{13} \mathrm{C}\left\{{ }^{\mathrm{H}} \mathrm{H}\right\} \mathrm{NMR}, 126 \mathrm{MHz}, \mathrm{CD}_{2} \mathrm{Cl}_{2}$}

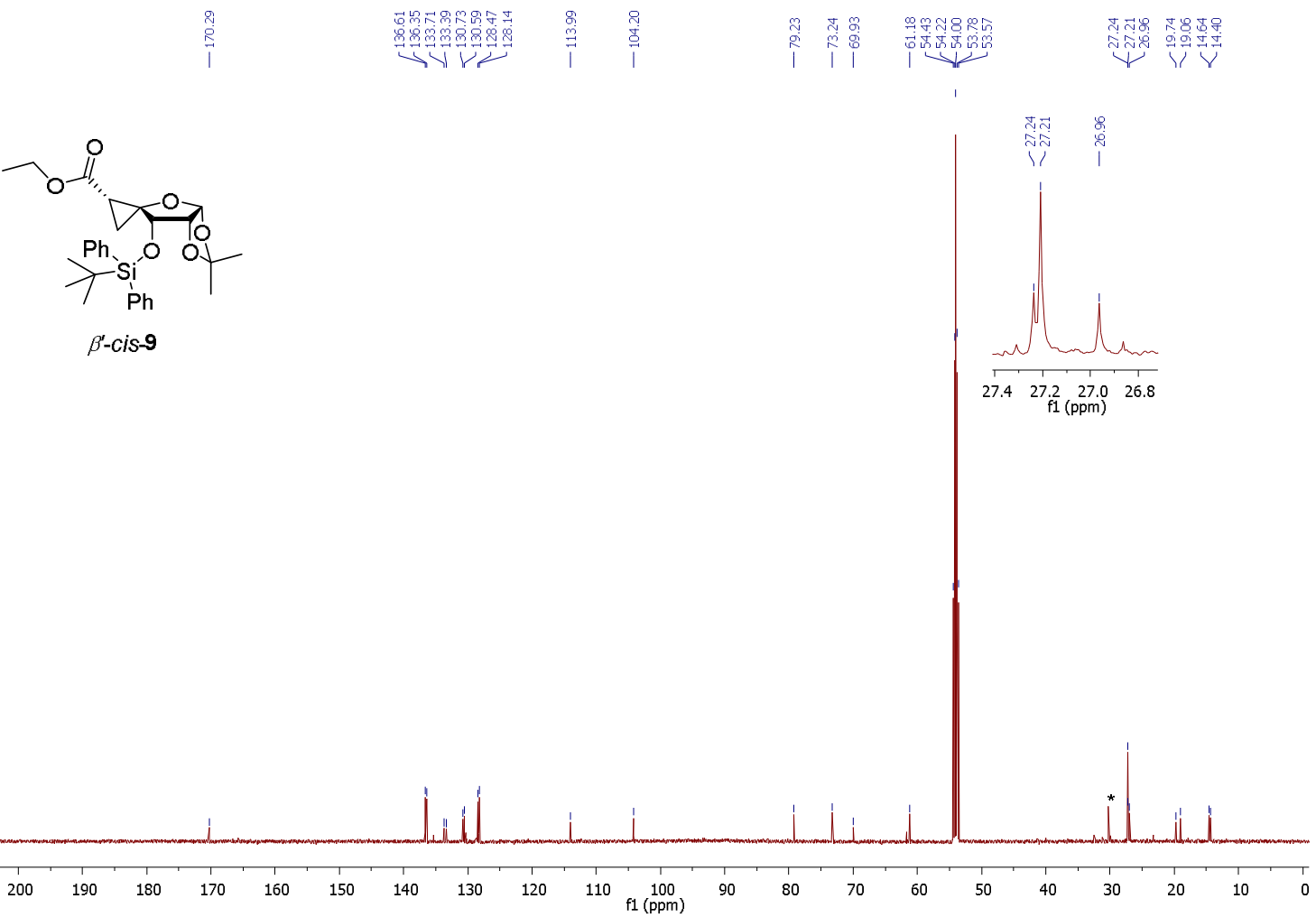




\section{${ }^{1} \mathrm{H} \mathrm{NMR}, 400 \mathrm{MHz}, \mathrm{CDCl}_{3}$}

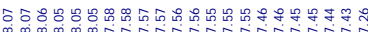

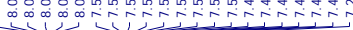

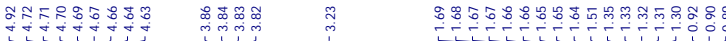<smiles>COC1OC2(C)OC1CC2OC(=O)c1ccccc1</smiles>

$\beta^{\prime}$-trans-C

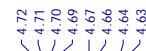

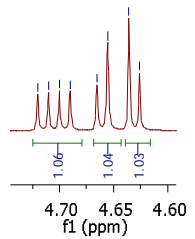

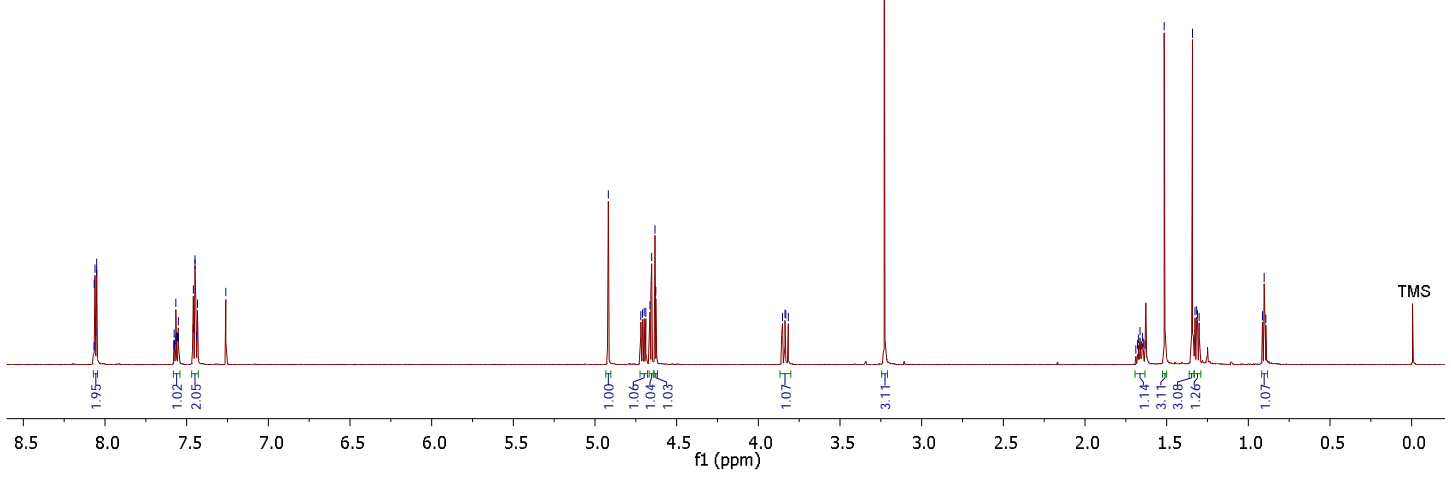

${ }^{13} \mathrm{C}^{\mathrm{l}}{ }^{\mathrm{H}} \mathrm{H}$ NMR, $100 \mathrm{MHz}, \mathrm{CDCl}_{3}$

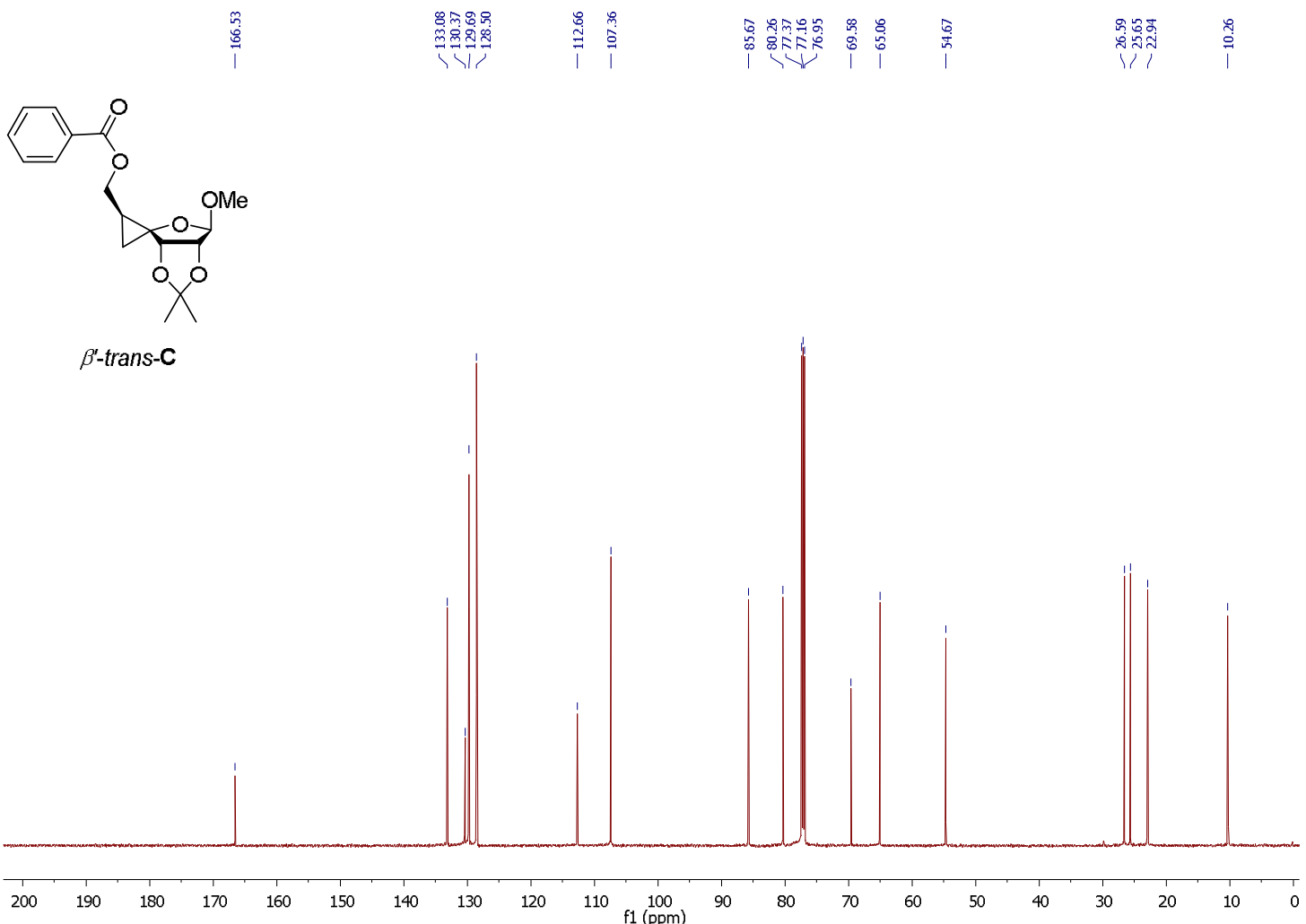


${ }^{1} \mathrm{H}$ NMR, $400 \mathrm{MHz}, \mathrm{CDCl}_{3}$

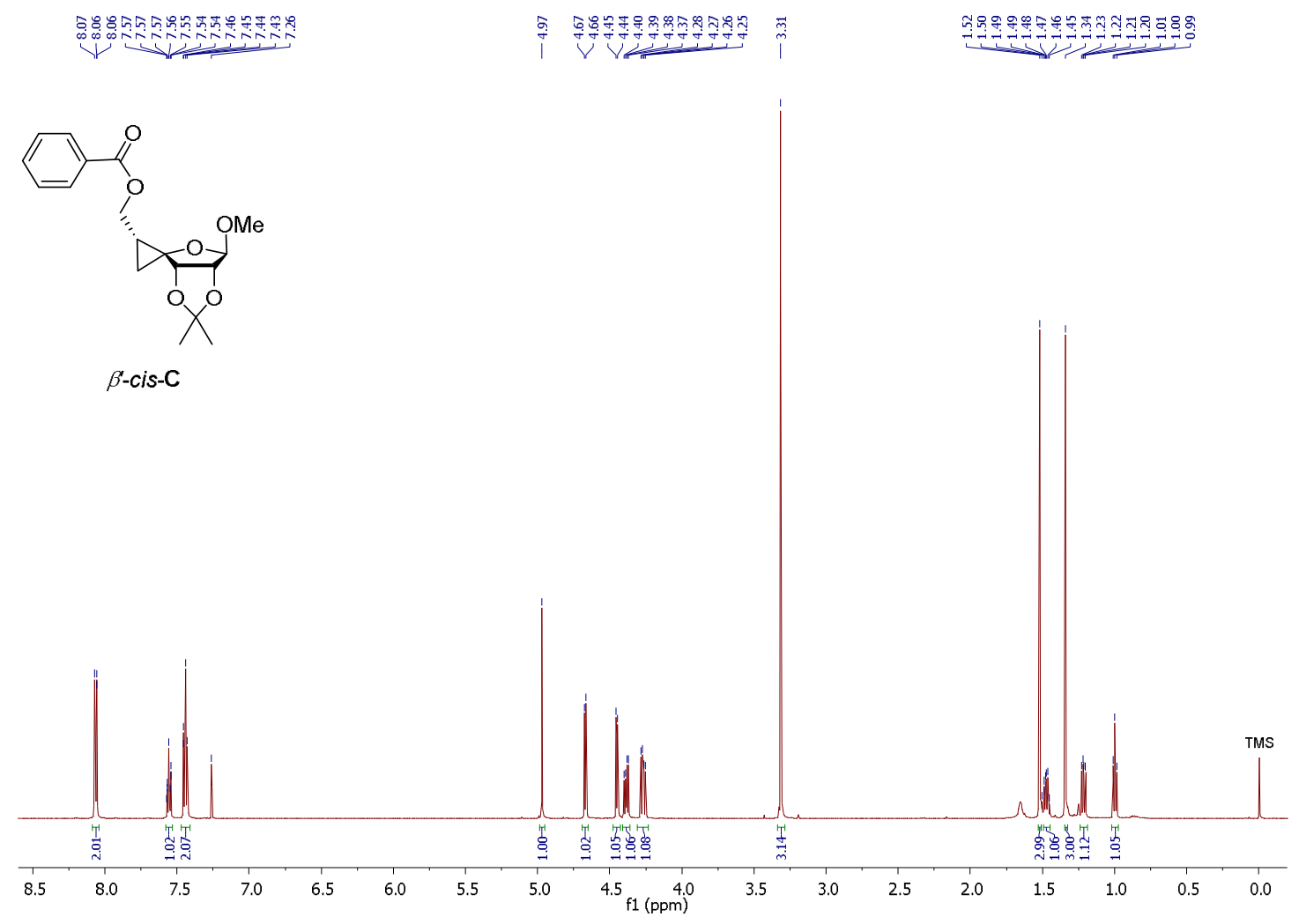

$\left.{ }^{13} \mathrm{C}^{1} \mathrm{H}\right\} \mathrm{NMR}, 100 \mathrm{M} \mathrm{Hz}, \mathrm{CDCl}_{3}$

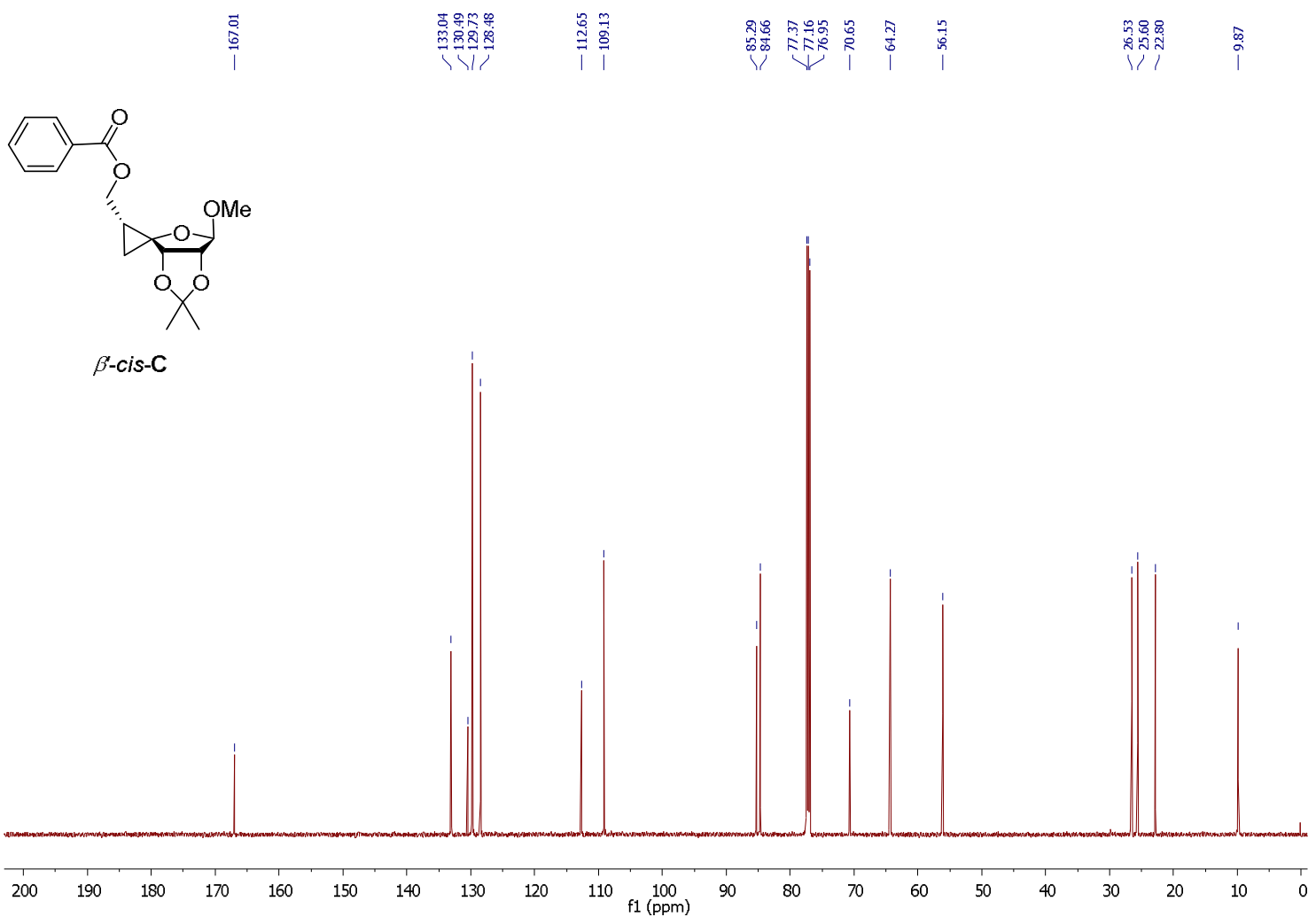


${ }^{1} \mathrm{H}$ NMR, $400 \mathrm{MHz}, \mathrm{CDCl}_{3}$

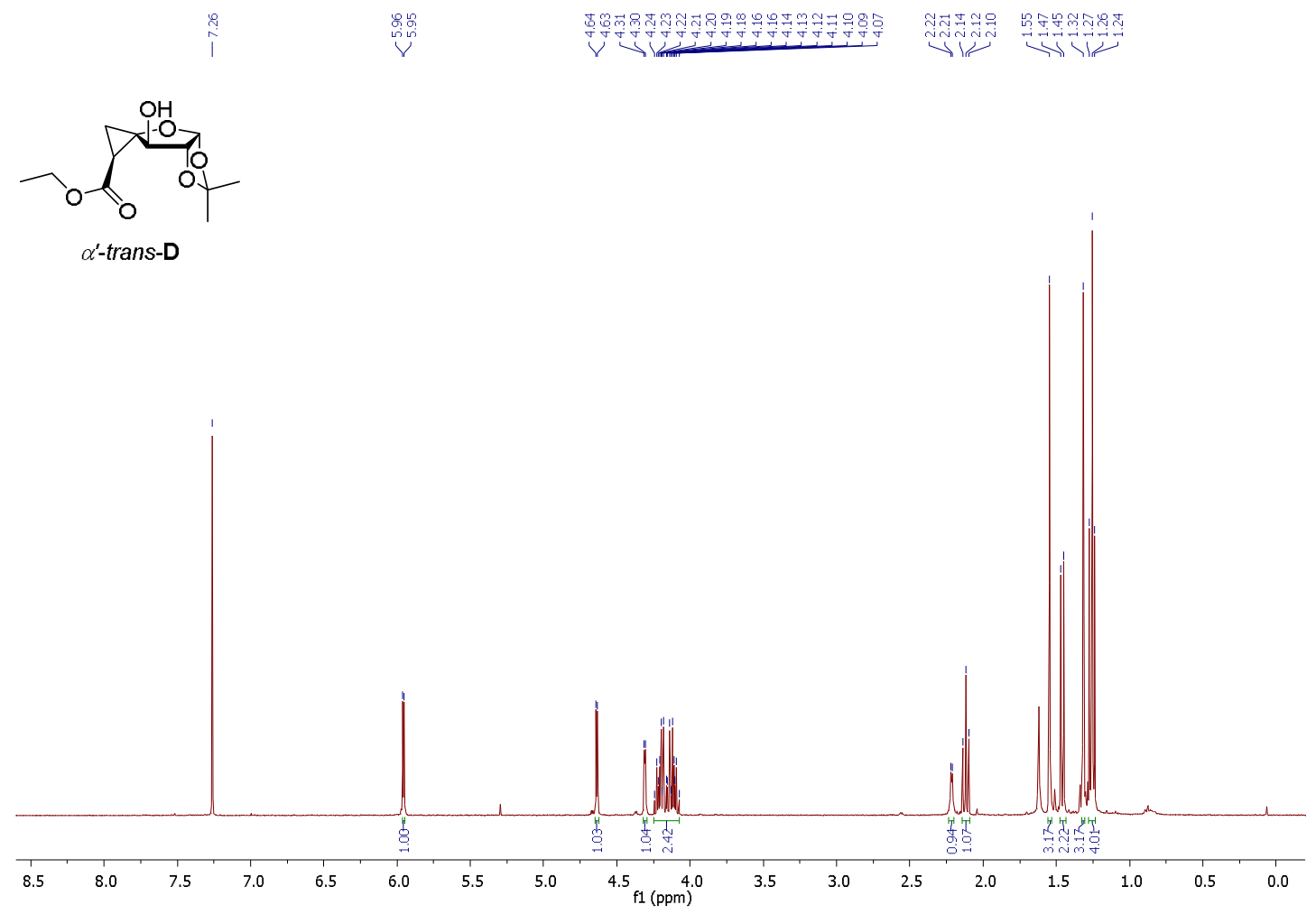

${ }^{13} \mathrm{C}\left\{{ }^{1} \mathrm{H}\right\} \mathrm{NMR}, 100 \mathrm{M} \mathrm{Hz}, \mathrm{CDCl}_{3}$

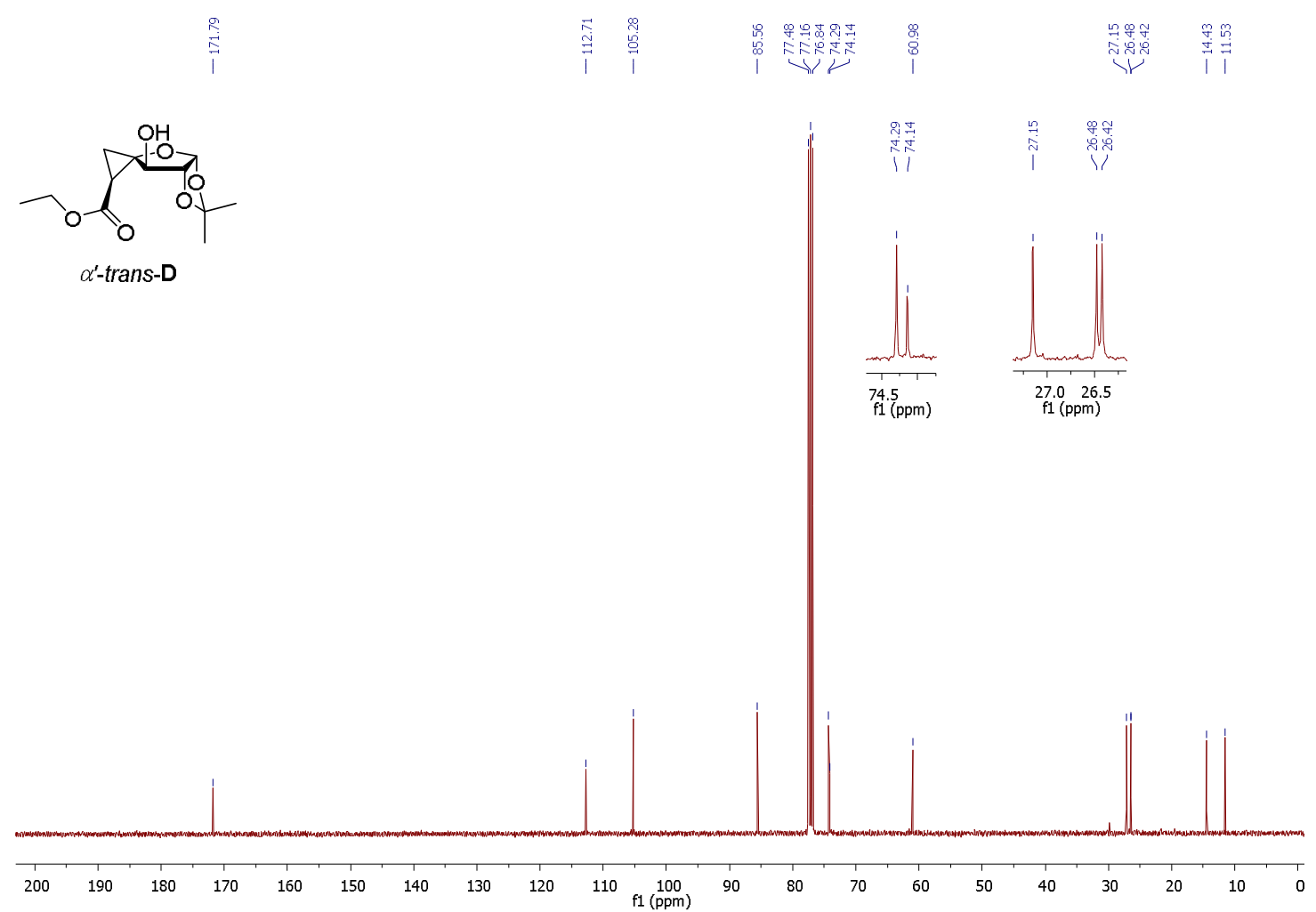

S-32 
${ }^{1} \mathrm{H}$ NMR, $400 \mathrm{MHz}_{,} \mathrm{CDCl}_{3}$

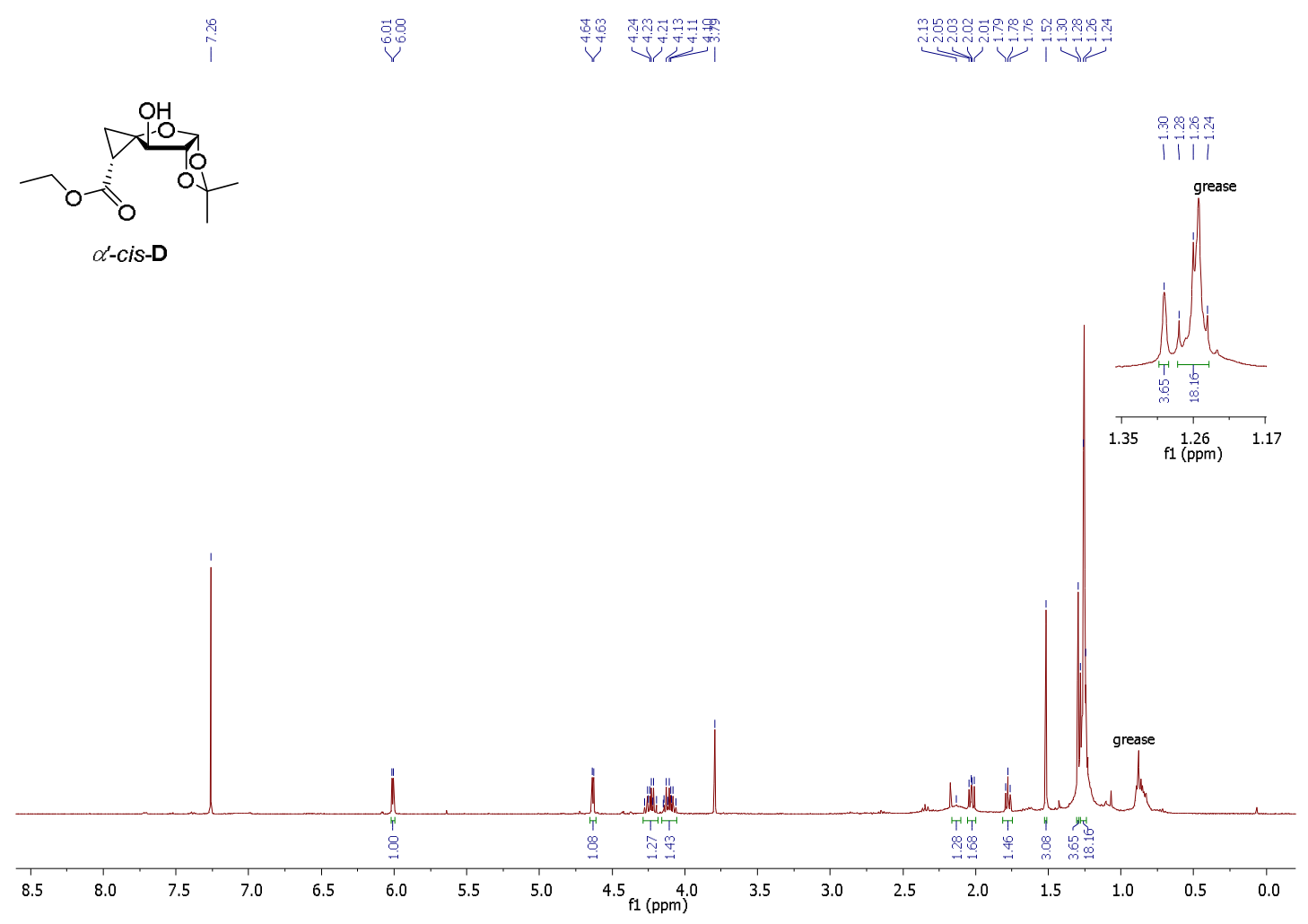

${ }^{13} \mathrm{C}\left\{{ }^{\mathrm{H}} \mathrm{H}\right\} \mathrm{NMR}, 126 \mathrm{MHz}, \mathrm{CDCl}_{3}$

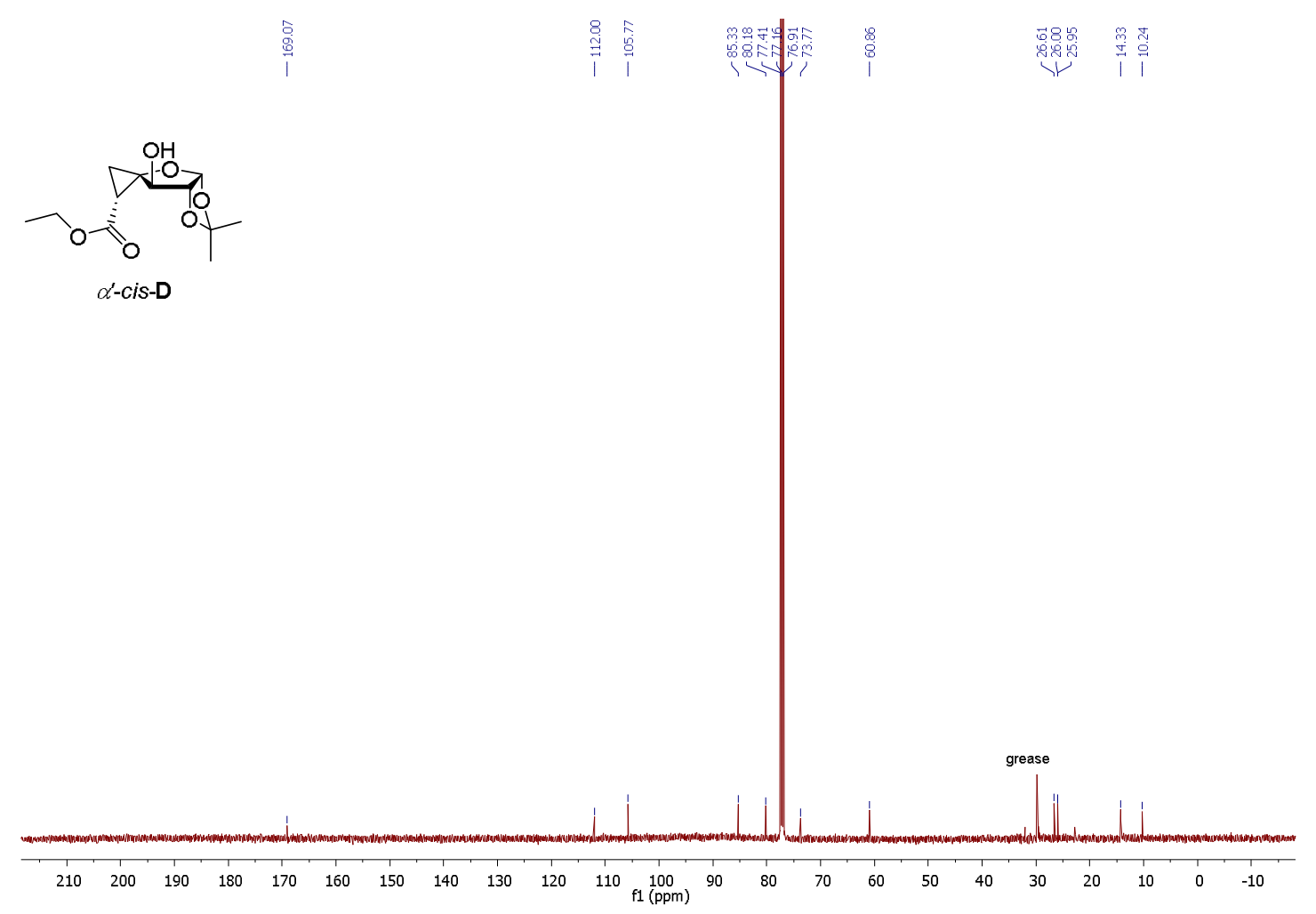

S-33 
${ }^{1} \mathrm{H} \mathrm{NMR}, 400 \mathrm{MHz}, \mathrm{CD}_{2} \mathrm{Cl}_{2}$

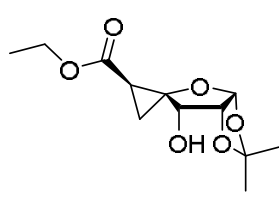

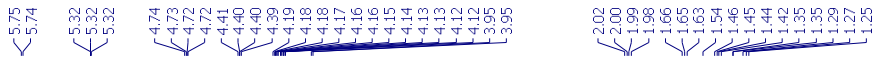

$\beta$-trans-E

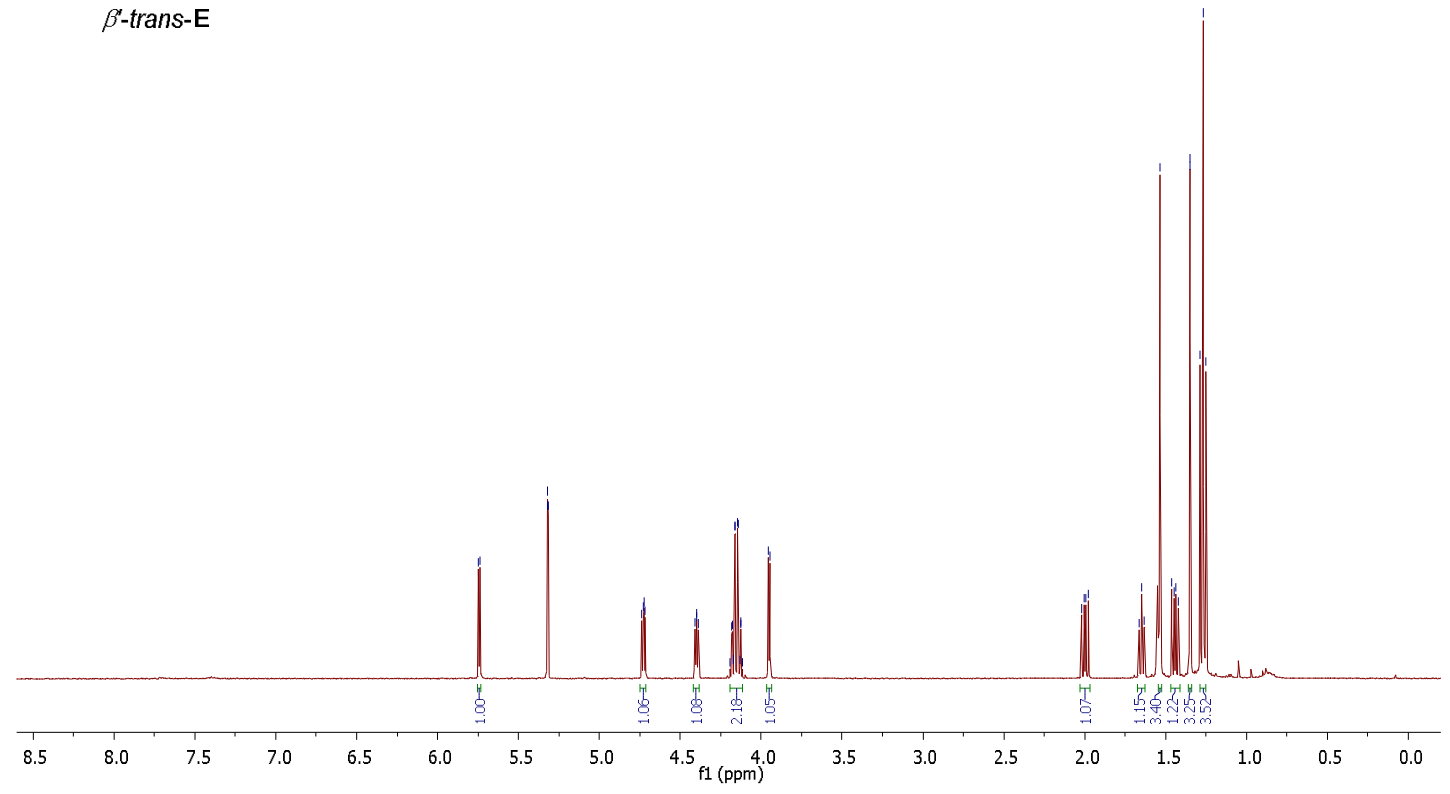

${ }^{13} \mathrm{C}\left\{{ }^{\mathrm{H}} \mathrm{H}\right\} \mathrm{NMR}, 100 \mathrm{MHz}, \mathrm{CD}_{2} \mathrm{Cl}_{2}$

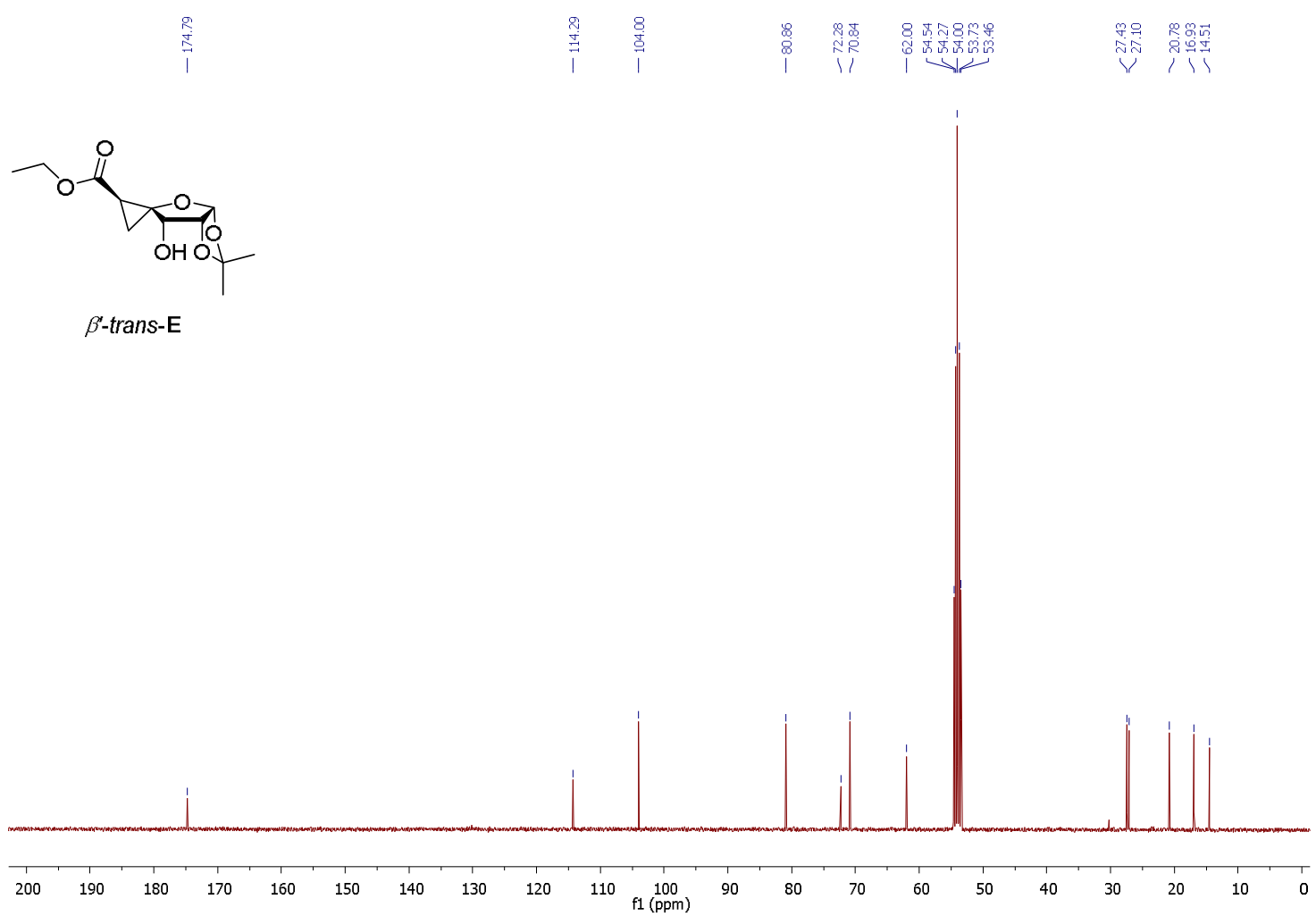

S-34 
${ }^{1} \mathrm{H}$ NMR, $400 \mathrm{MHz}, \mathrm{CD}_{2} \mathrm{Cl}_{2}$

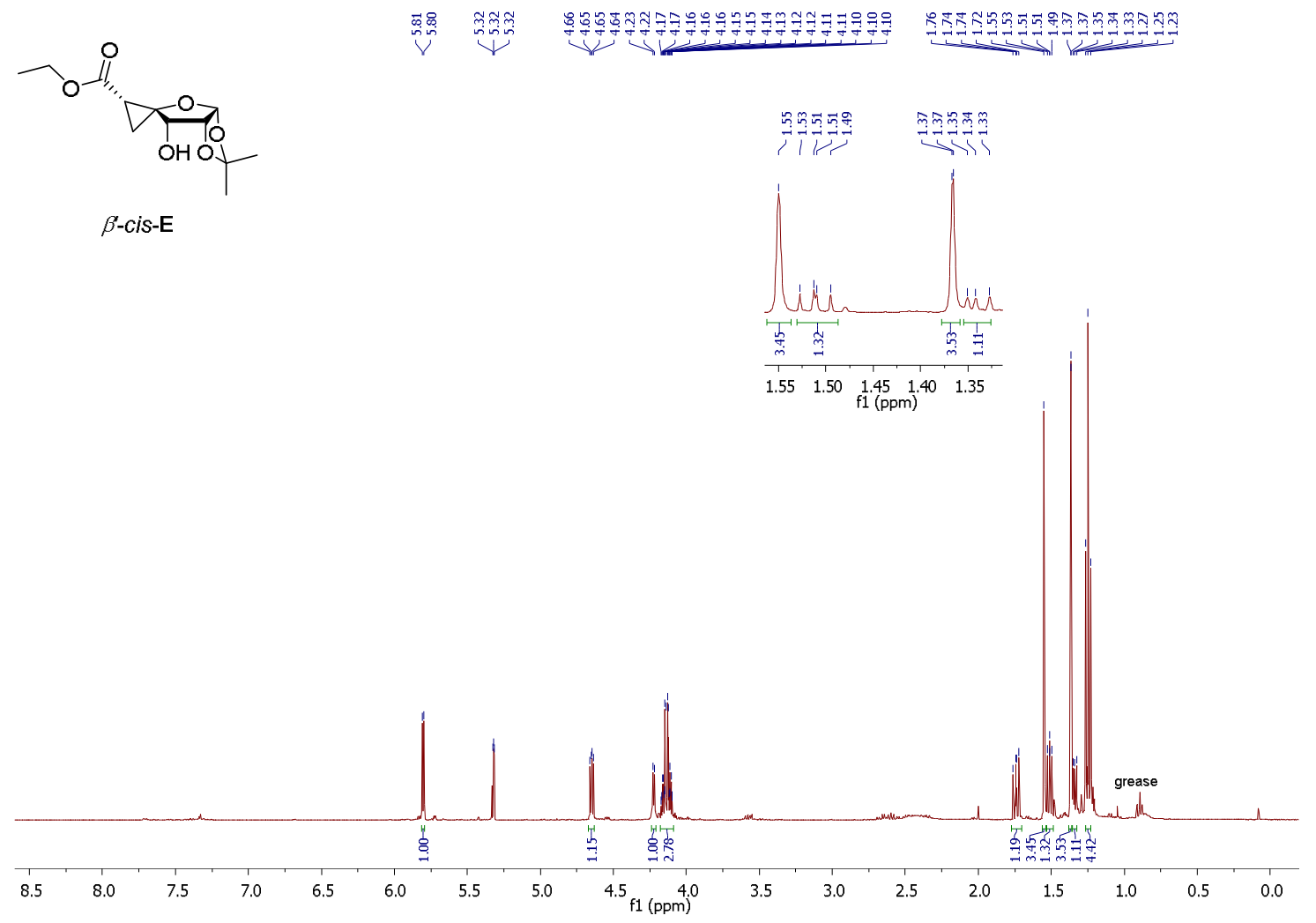

${ }^{13} \mathrm{C}\left\{{ }^{1} \mathrm{H}\right\} \mathrm{NMR}, 75 \mathrm{MHz}, \mathrm{CD}_{2} \mathrm{Cl}_{2}$

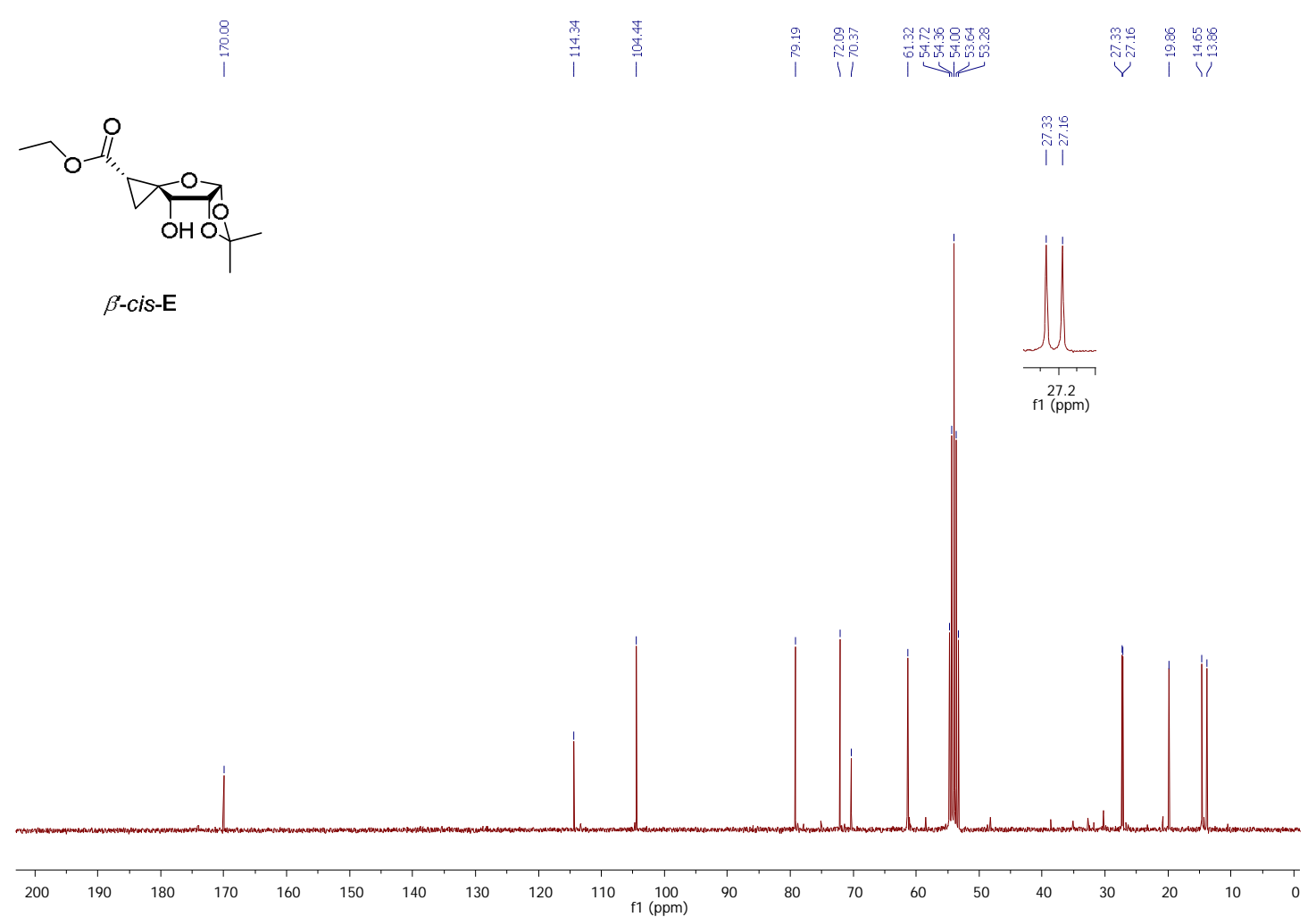

S-35 


\section{${ }^{1} \mathrm{H}$ NMR, $400 \mathrm{MHz}, \mathrm{CDCl}_{3}$}

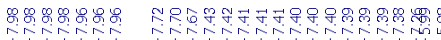
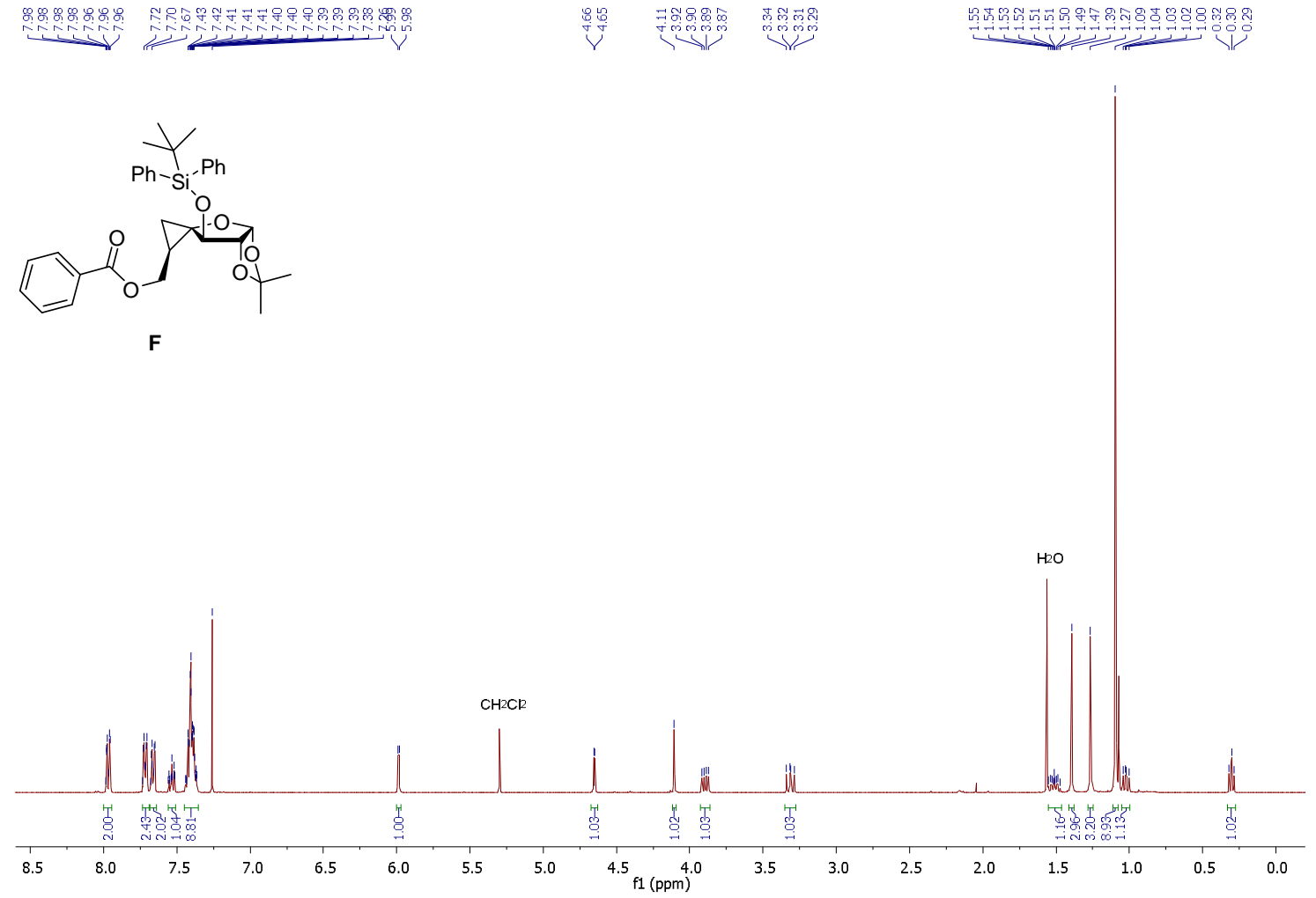

\section{$\left.{ }^{13} \mathrm{C}^{\mathrm{l}}{ }^{\mathrm{H}} \mathrm{H}\right\} \mathrm{NMR}, 100 \mathrm{M} \mathrm{Hz}, \mathrm{CDCl}_{3}$}

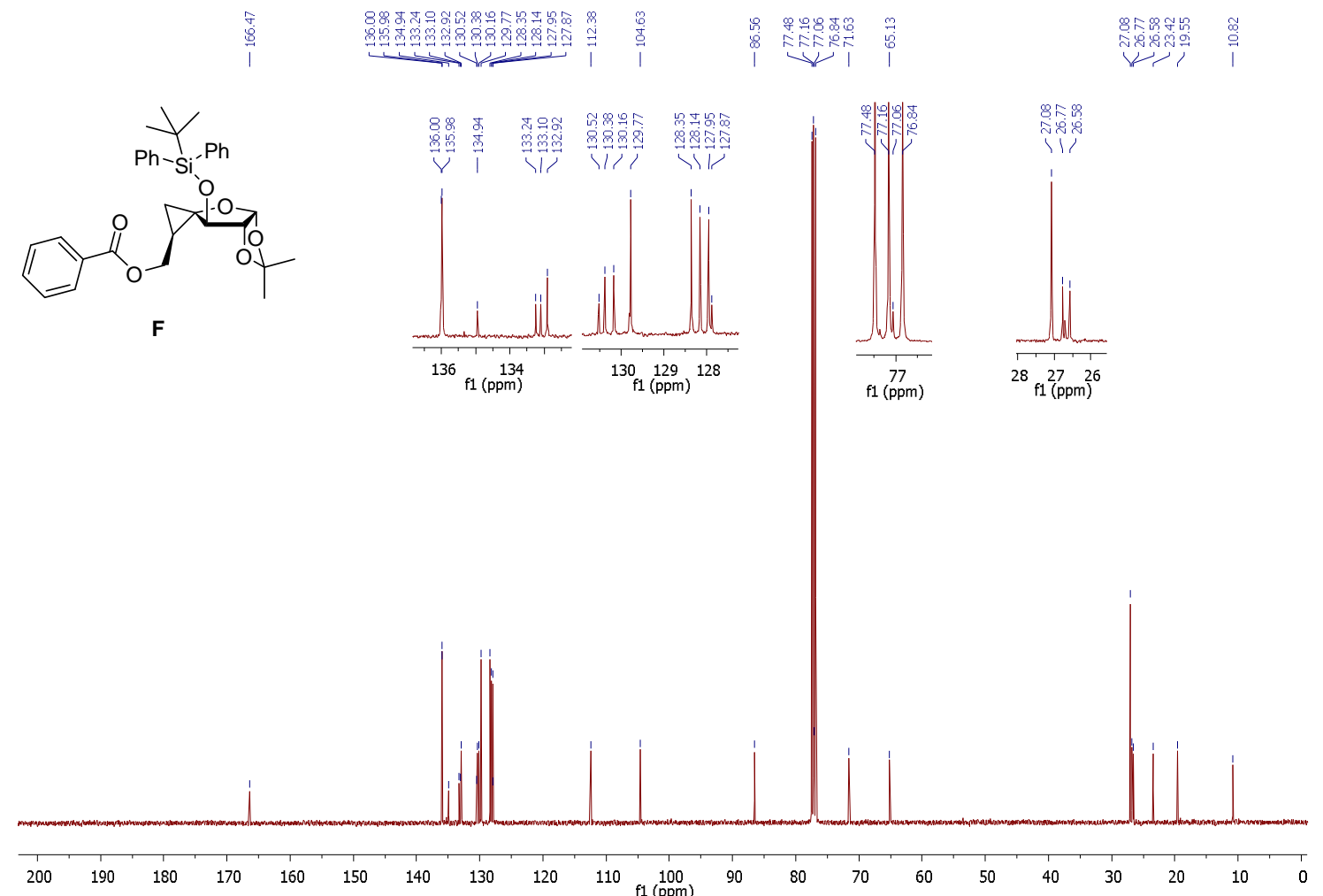


${ }^{1} \mathrm{H}$ NMR, $400 \mathrm{MHz}, \mathrm{CDCl}_{3}$

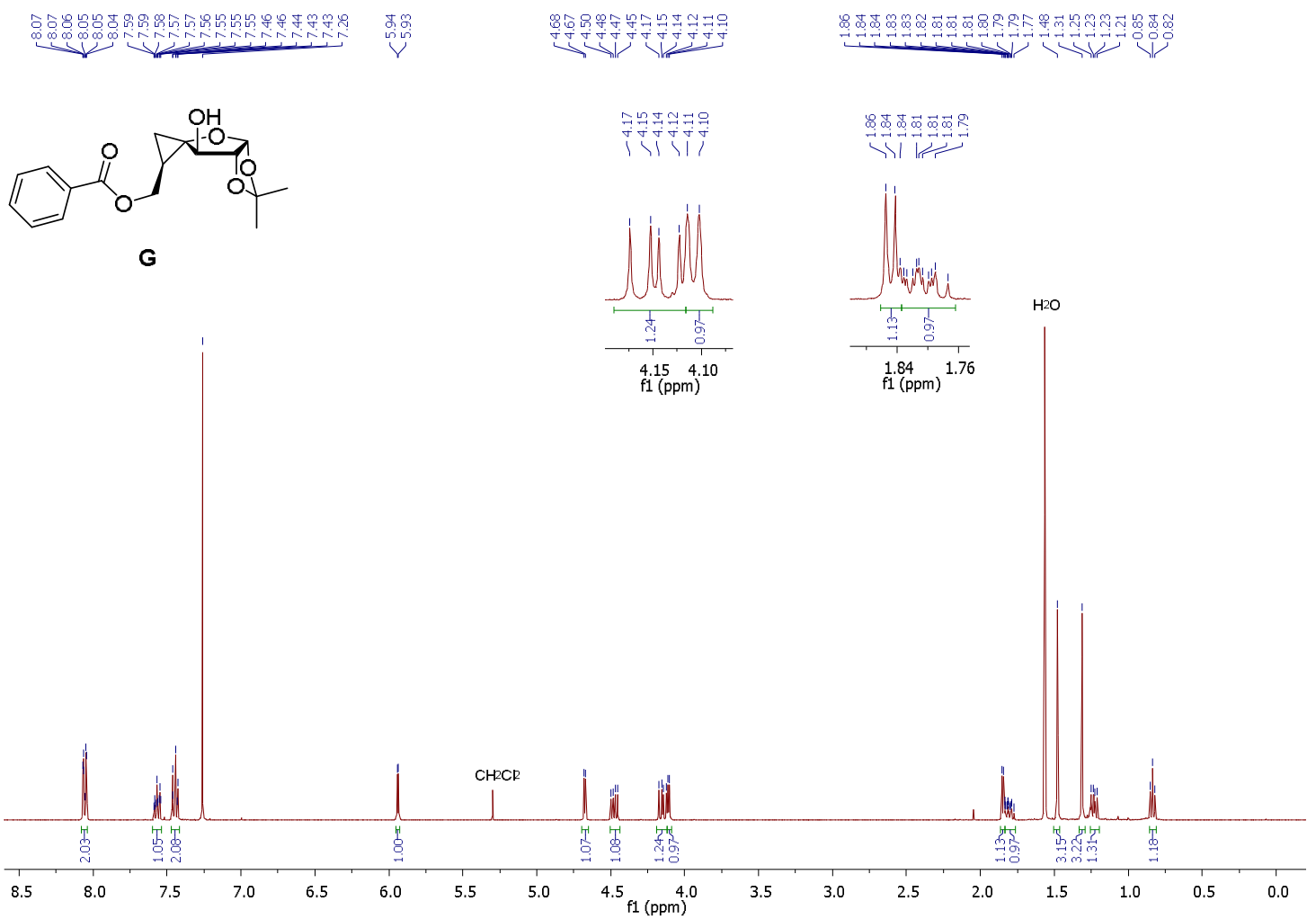

${ }^{13} \mathrm{C}\left\{{ }^{-} \mathrm{H}\right\}$ NMR, $100 \mathrm{M} \mathrm{Hz}, \mathrm{CDCl}_{3}$
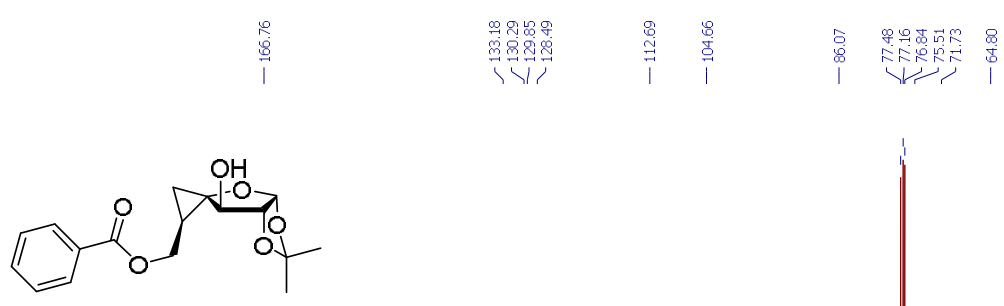

G

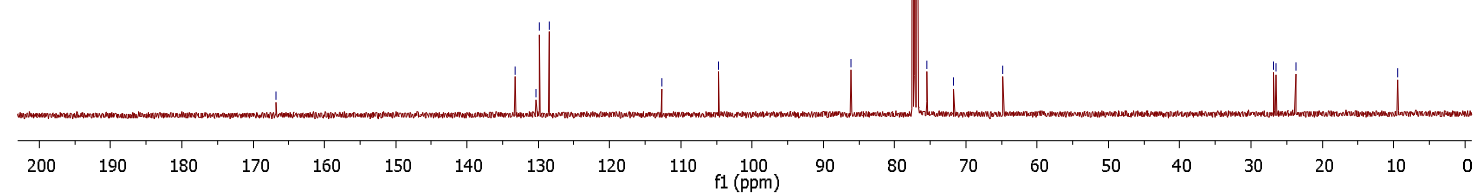


${ }^{1} \mathrm{H}$ NMR, $400 \mathrm{MHz}, \mathrm{CDCl}_{3}$

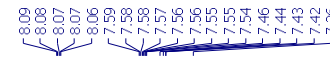

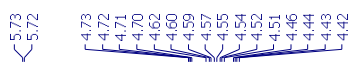

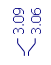

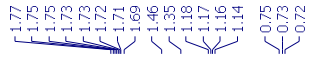

1.

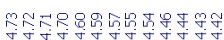

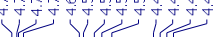

H
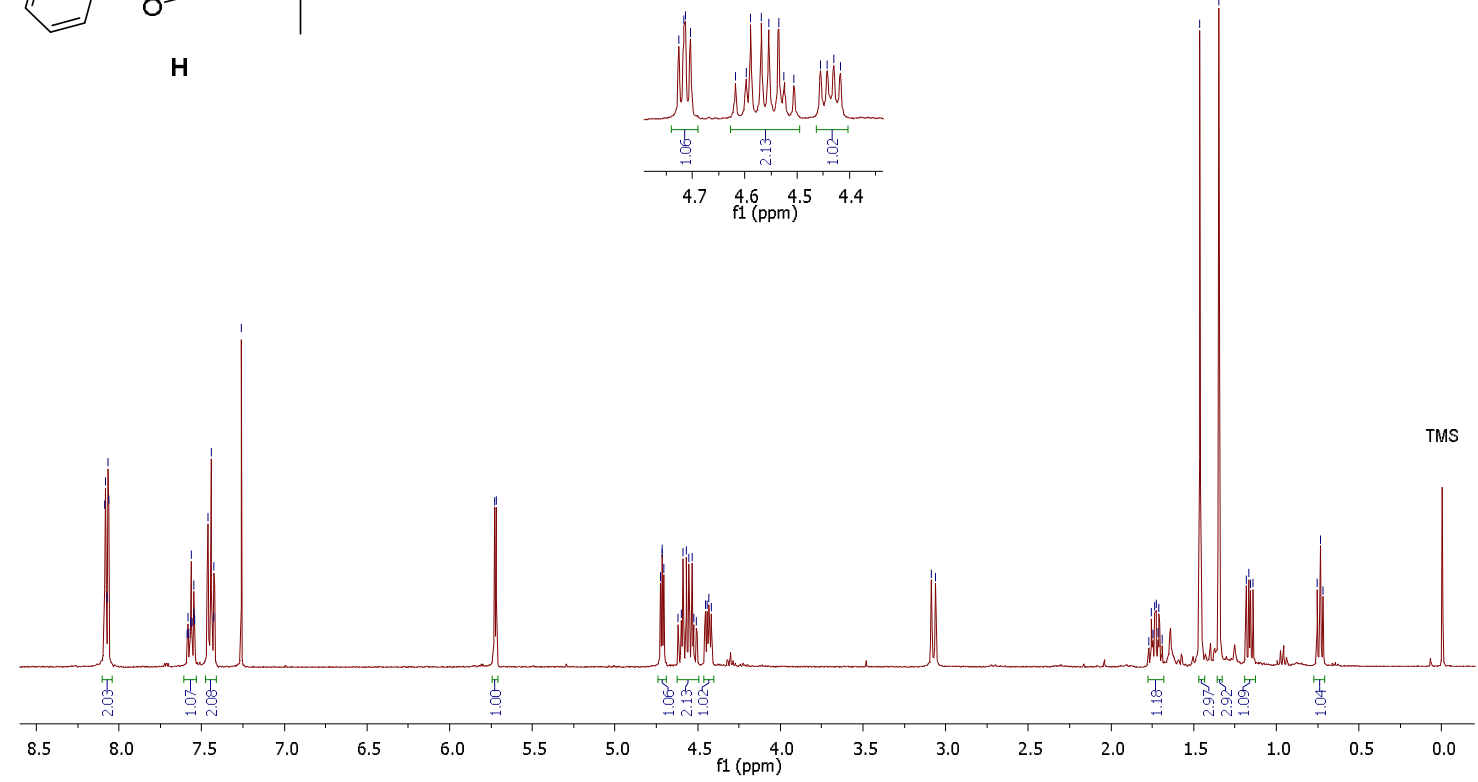

${ }^{13} \mathrm{C}\left\{{ }^{1} \mathrm{H}\right\} \mathrm{NMR}, 100 \mathrm{MHz}, \mathrm{CDCl}_{3}$

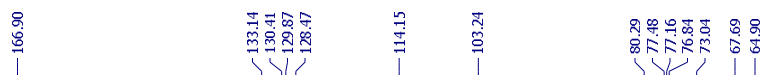

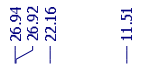

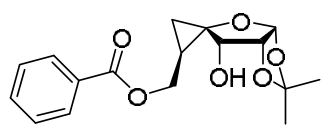

H
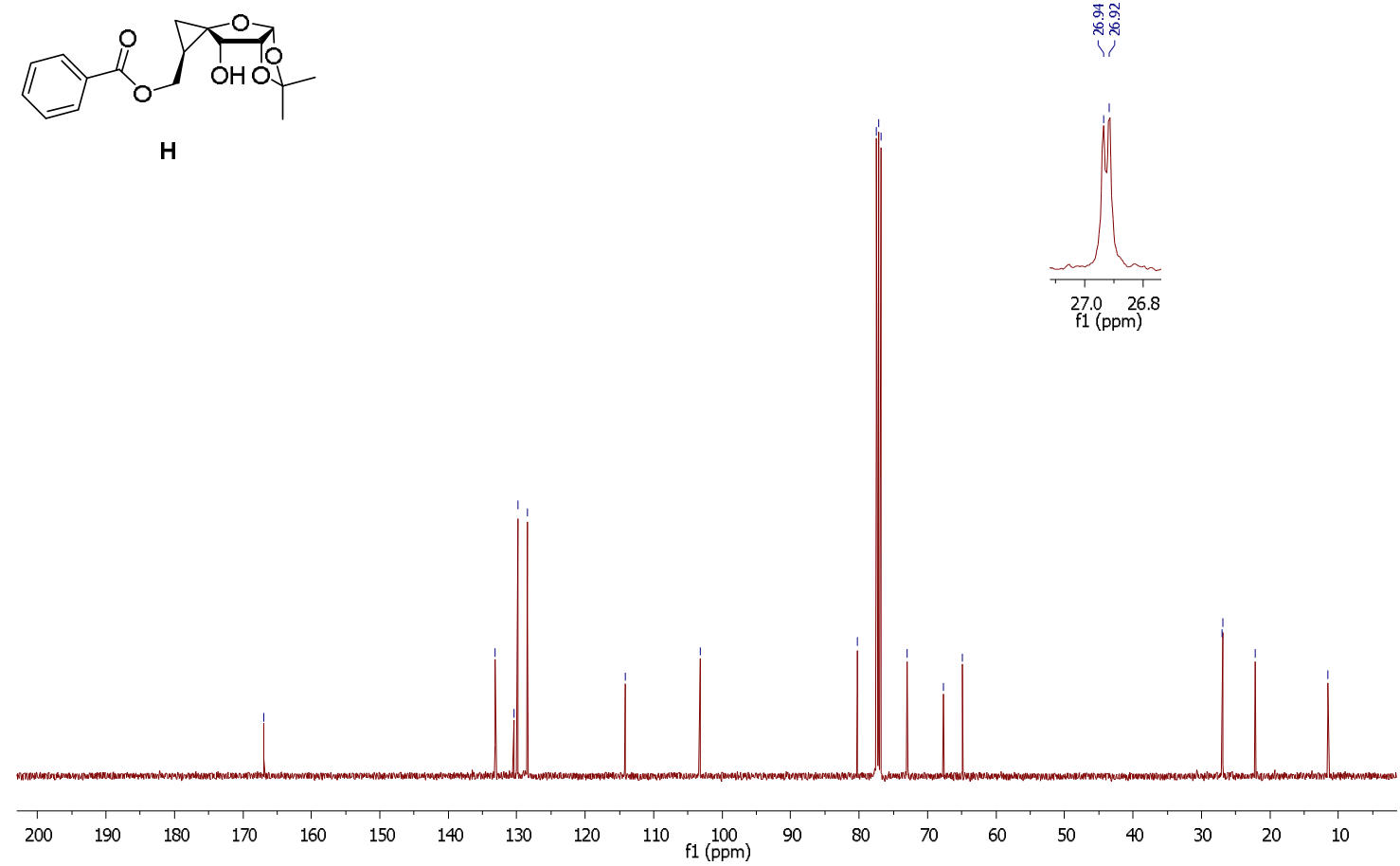

S-38 
${ }^{1} \mathrm{H} \mathrm{NMR}, 300 \mathrm{MHz}, \mathrm{CDCl}_{3}$ (contains $20 \%$ of the minor isomer)

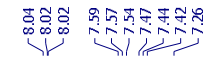

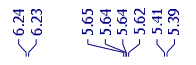

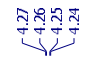

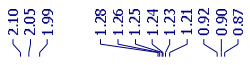

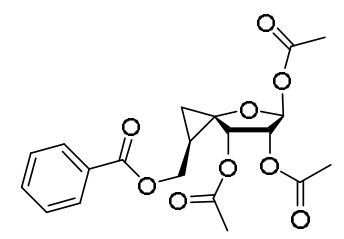

I (major)

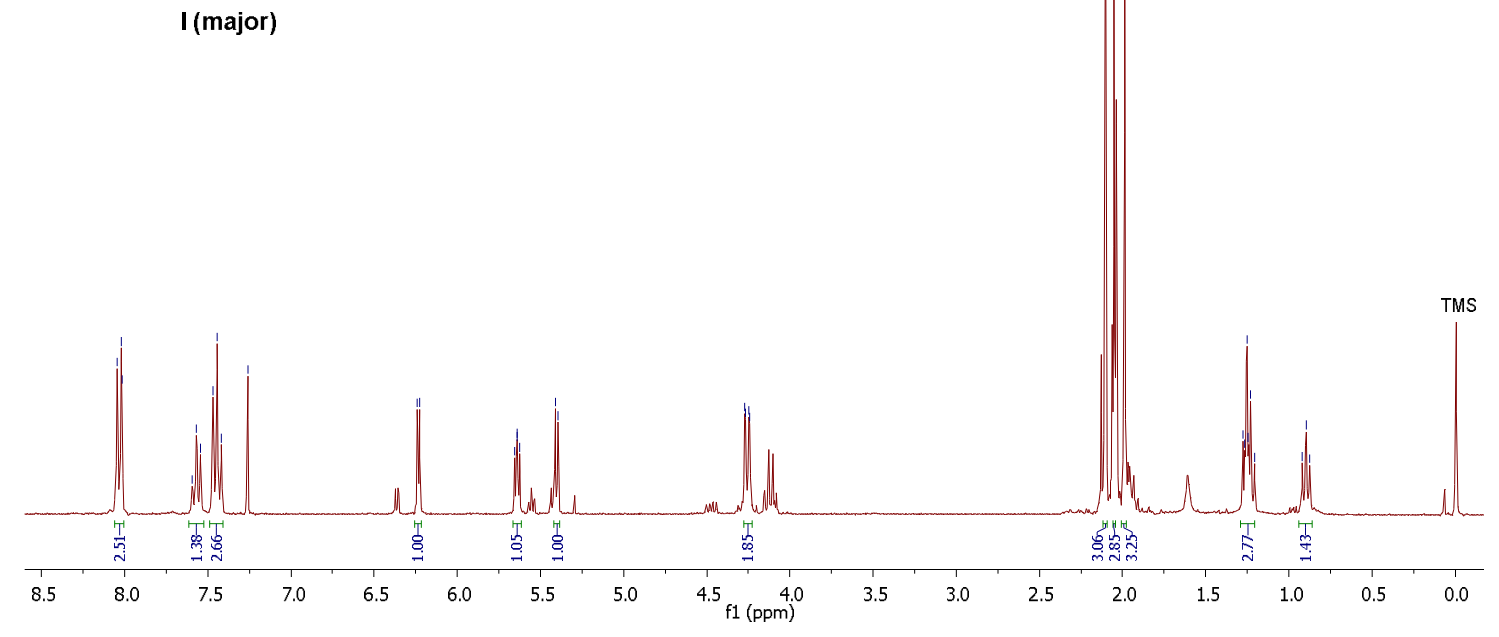

${ }^{13} \mathrm{C}\left\{{ }^{1} \mathrm{H}\right\} \mathrm{NMR}, 75 \mathrm{M} \mathrm{Hz}^{\mathrm{CDCl}} \mathrm{Cl}_{3}$ (contains $20 \%$ of the minor isomer)

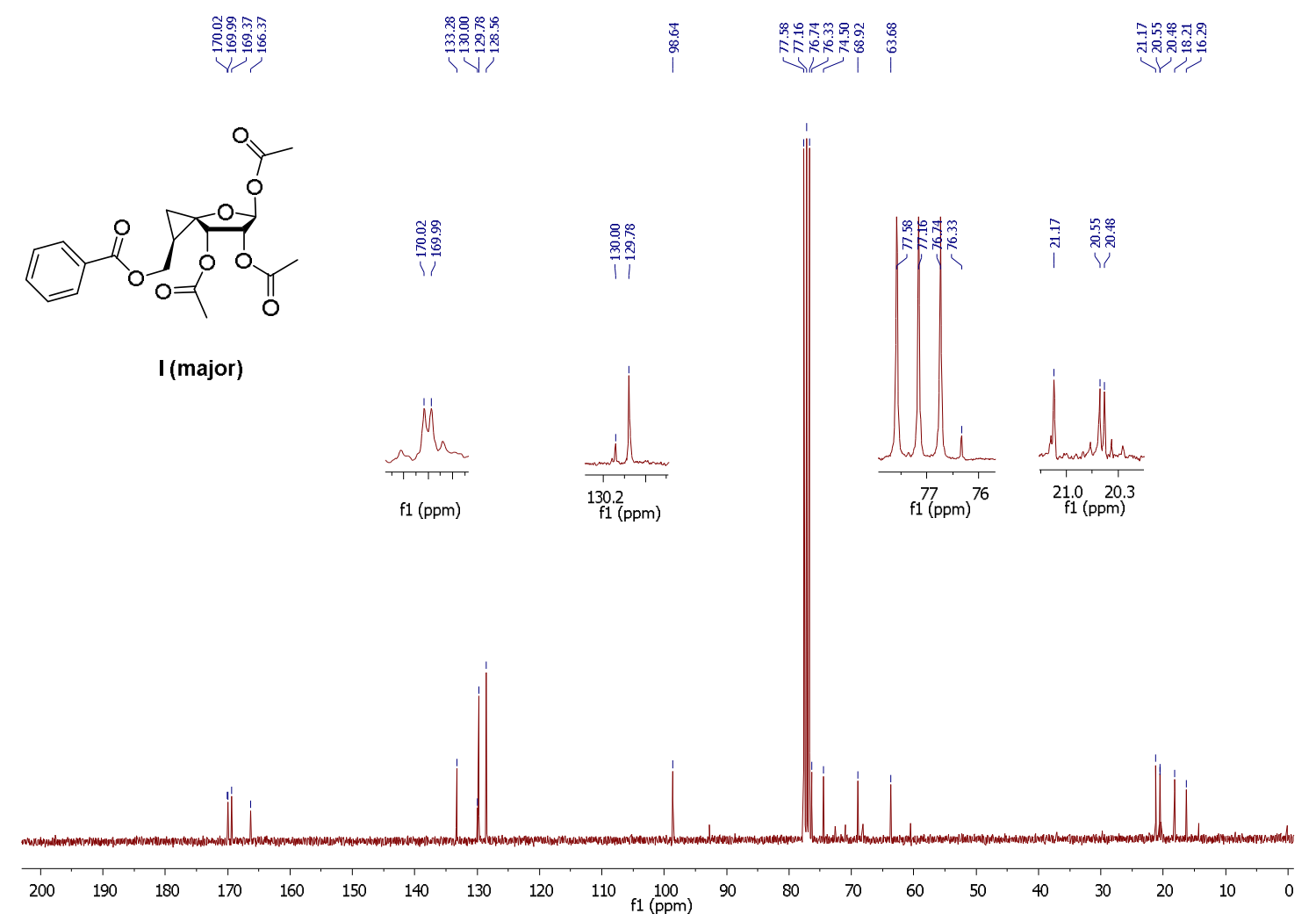

S-39 
${ }^{1} \mathrm{H}$ NMR, $400 \mathrm{MHz}$, Methanol-d

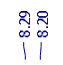

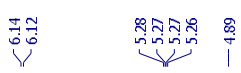

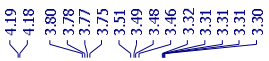

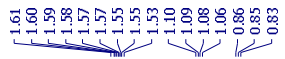

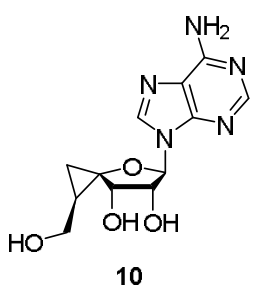

10

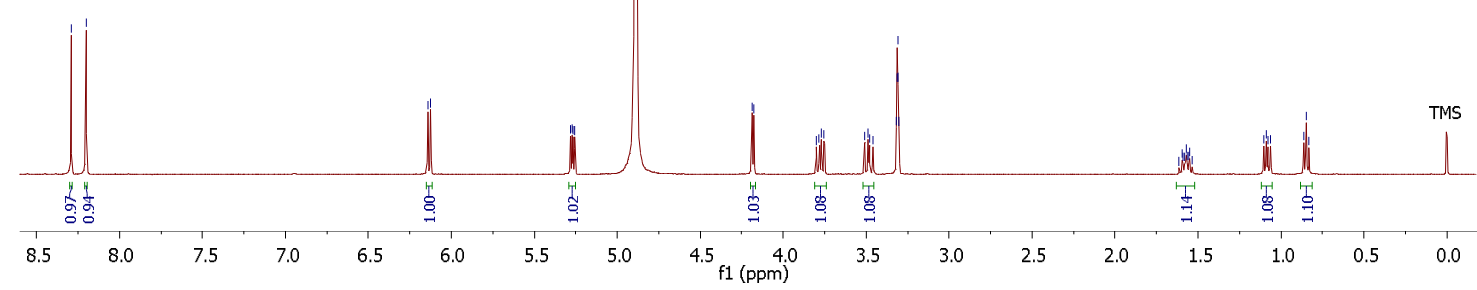

${ }^{13} \mathrm{C}\left\{{ }^{\mathrm{l}} \mathrm{H}\right\}$ NMR, $100 \mathrm{MHz}$, Methanol- $\mathrm{d}_{4}$

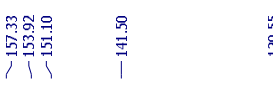

$\stackrel{5}{1}$<smiles>Nc1ncnc2c1ncn2C(O)C1CC1CO</smiles>

10

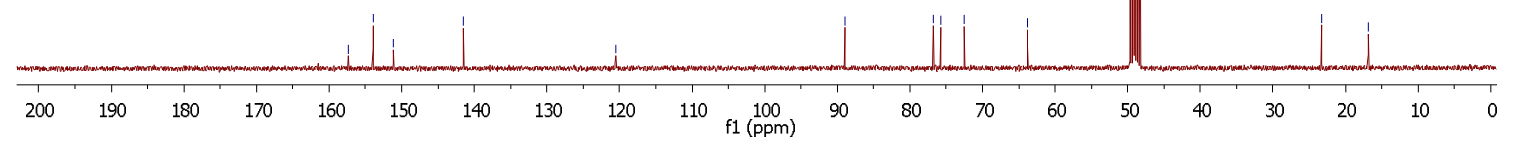




\section{${ }^{1} \mathrm{H}$ NMR, $400 \mathrm{MHz}, \mathrm{CDCl}_{3}$}

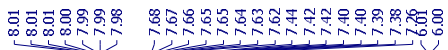

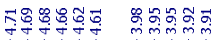

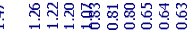
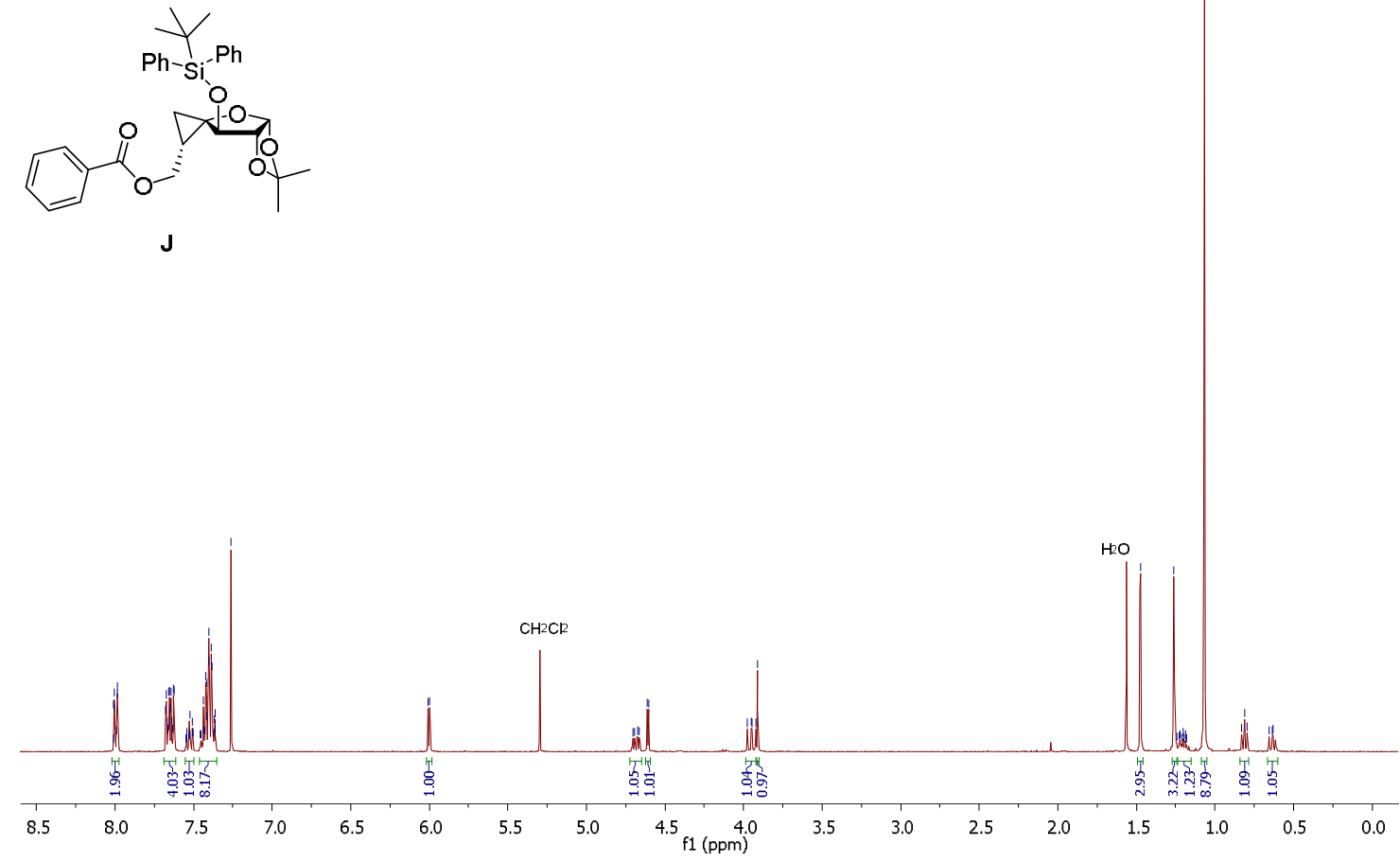

${ }^{13} \mathrm{C}\left\{{ }^{\mathrm{l}} \mathrm{H}\right\} \mathrm{NMR}, 100 \mathrm{MHz}, \mathrm{CDCl}_{3}$

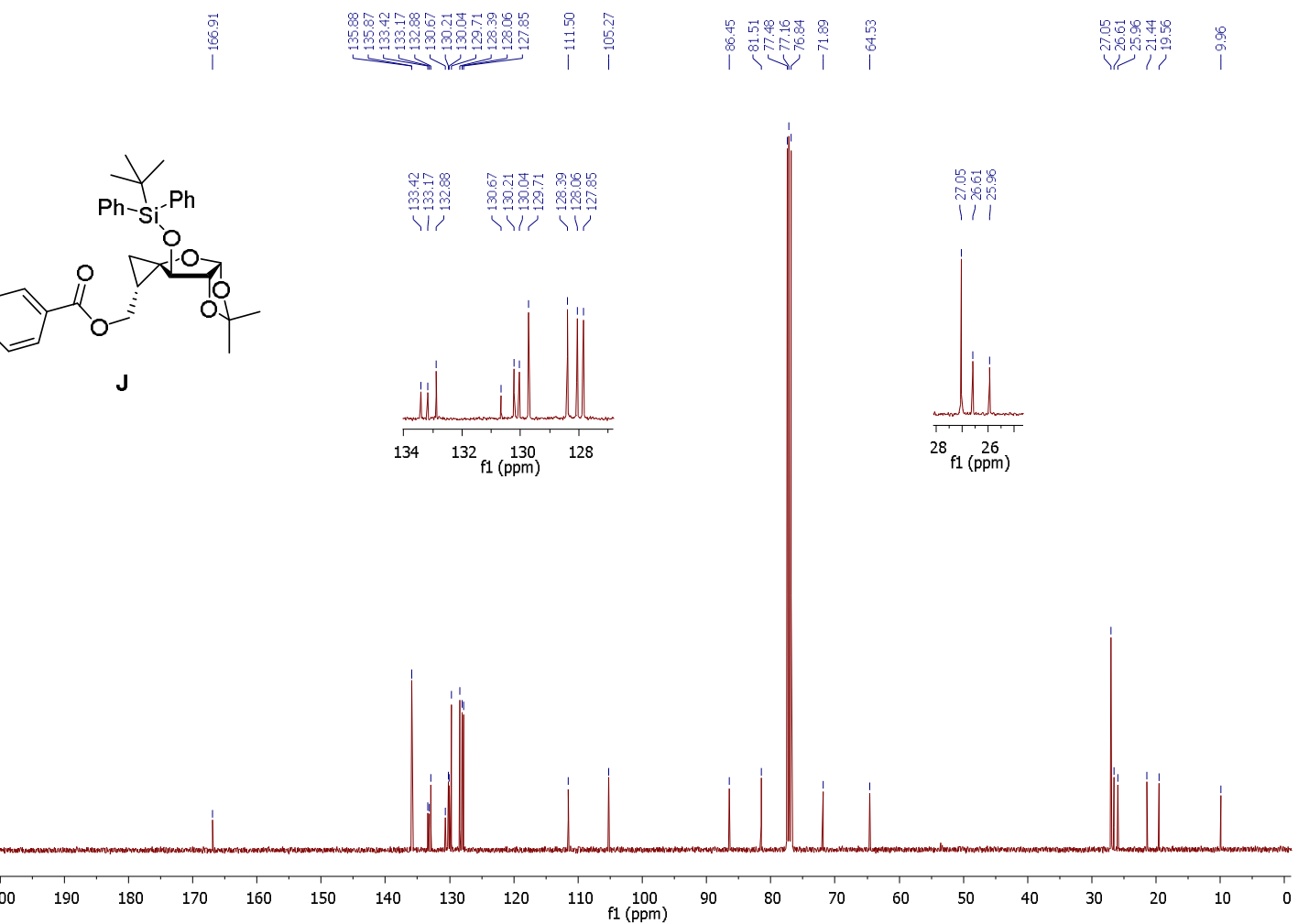


${ }^{1} \mathrm{H}$ NMR, $400 \mathrm{MHz}_{2} \mathrm{CDCl}_{3}$

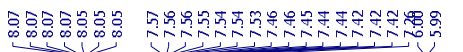

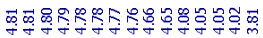

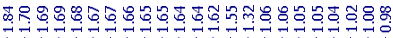

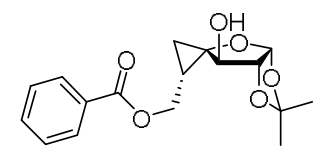

K

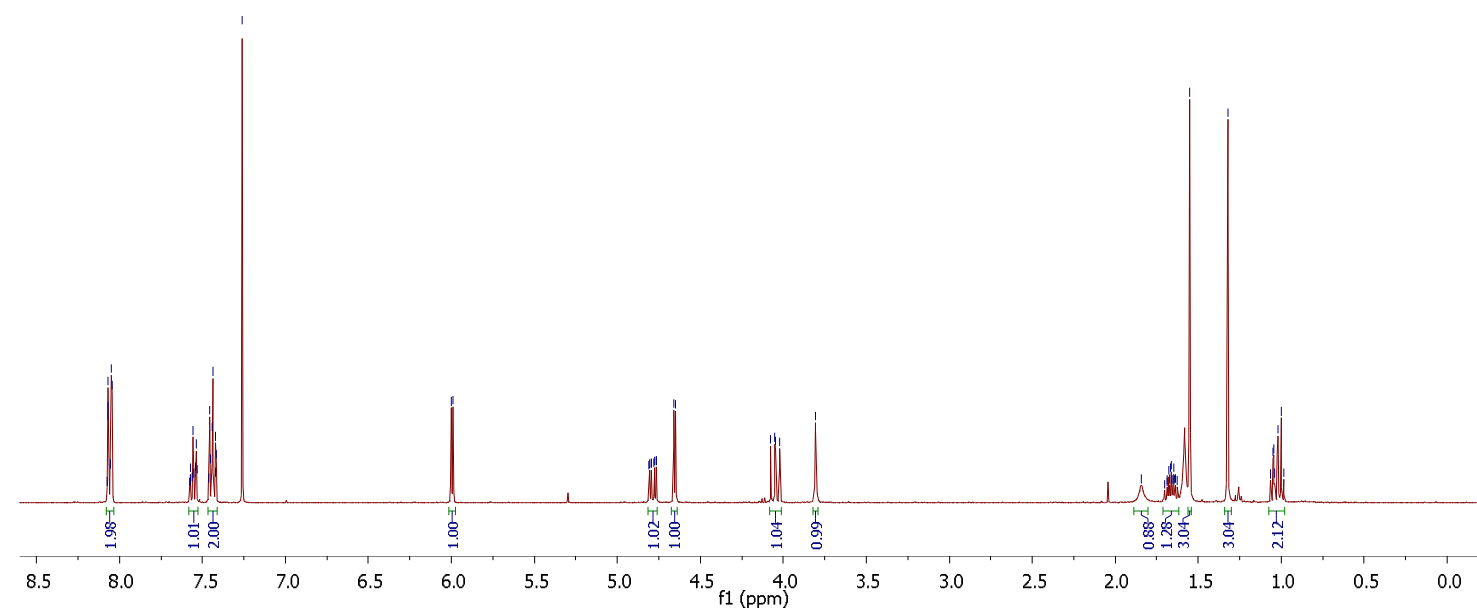

${ }^{13} \mathrm{C}\left\{{ }^{\mathrm{l}} \mathrm{H}\right\} \mathrm{NMR}, 75 \mathrm{M} \mathrm{Hz}^{\mathrm{CDCl}}{ }_{3}$
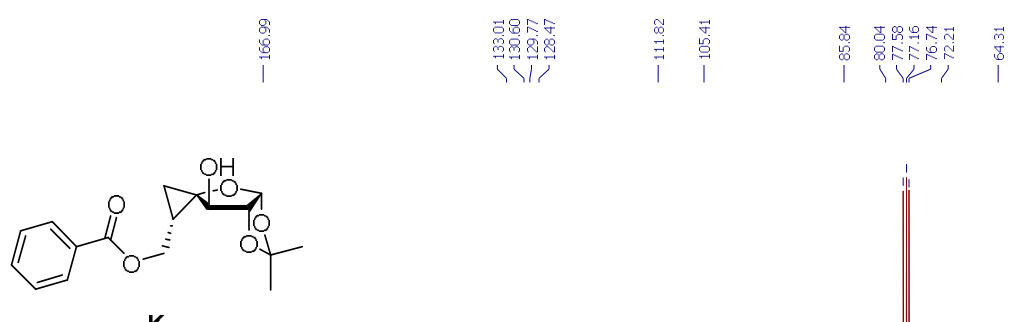

K

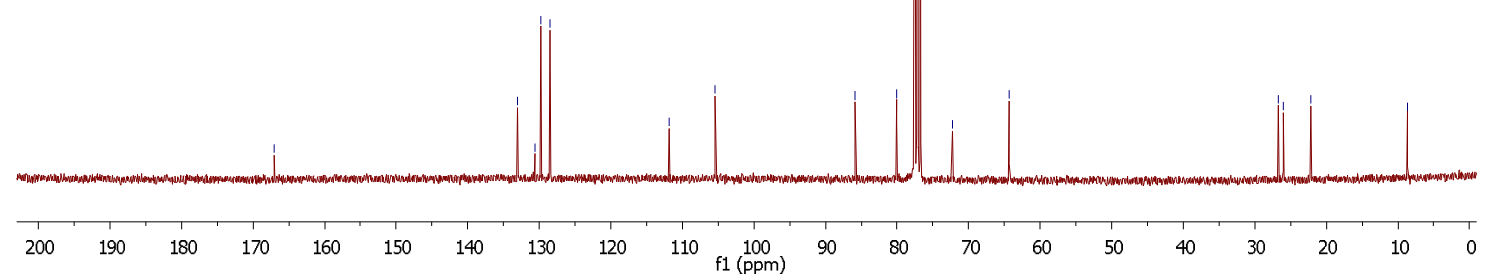




\section{${ }^{1} \mathrm{H}$ NMR, $400 \mathrm{MHz}, \mathrm{CD}_{2} \mathrm{Cl}_{2}$}

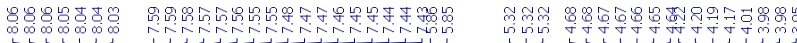

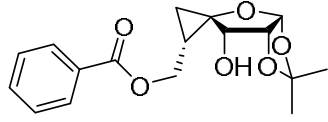

L

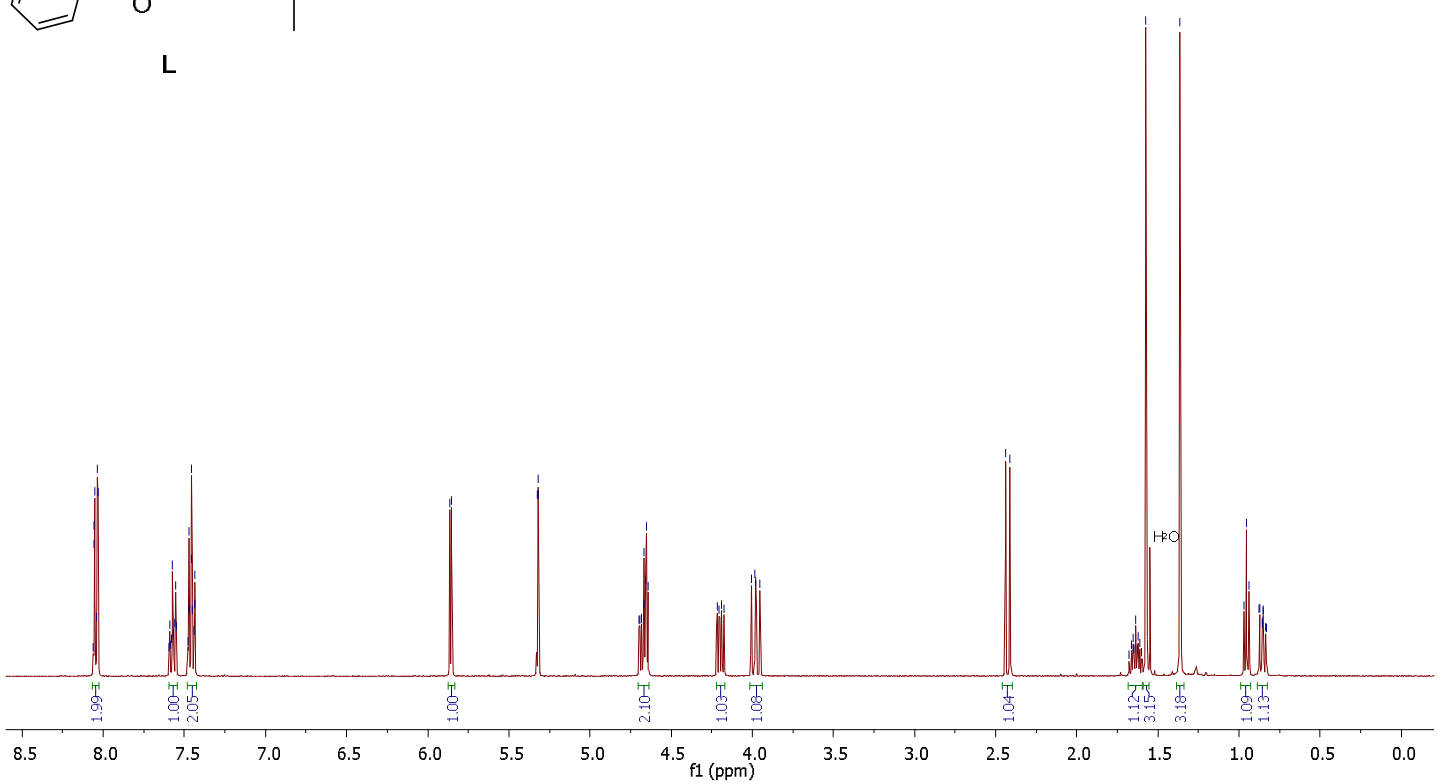

${ }^{13} \mathrm{C}\left\{\mathrm{H} H\right.$ NMR, $100 \mathrm{MHz}, \mathrm{CD}_{2} \mathrm{Cl}_{2}$

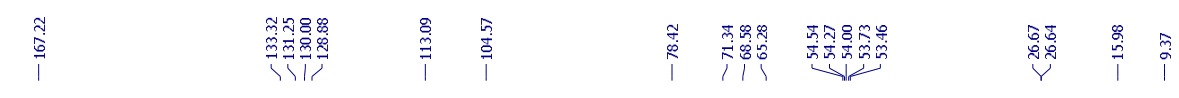

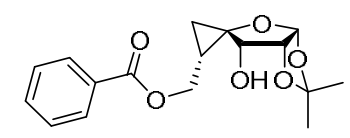

$\mathbf{L}$

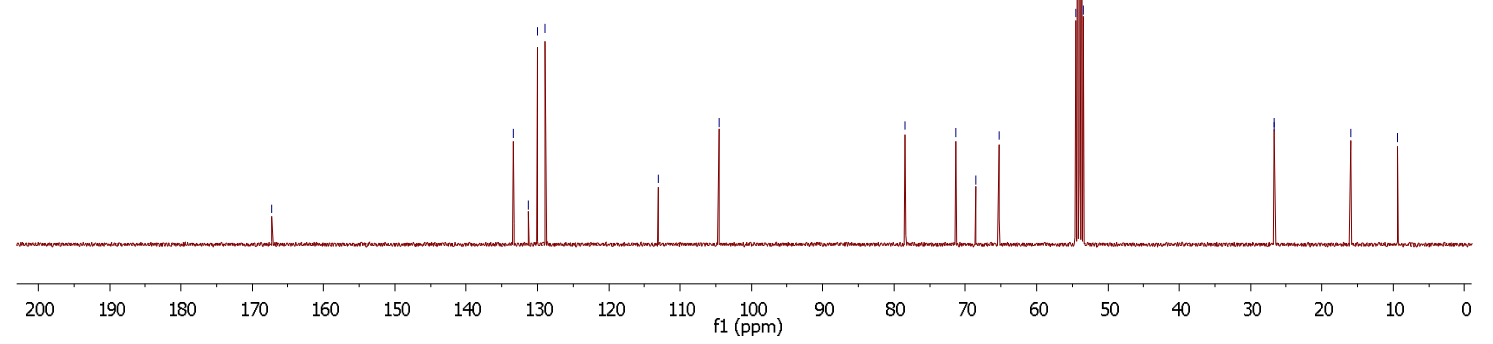


${ }^{1} \mathrm{H}$ NMR, $300 \mathrm{MHz}, \mathrm{CDCl}_{3}$ (contains $10 \%$ of the minor isomer)

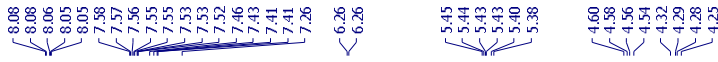

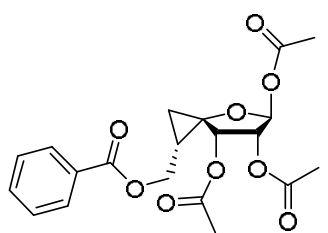

M
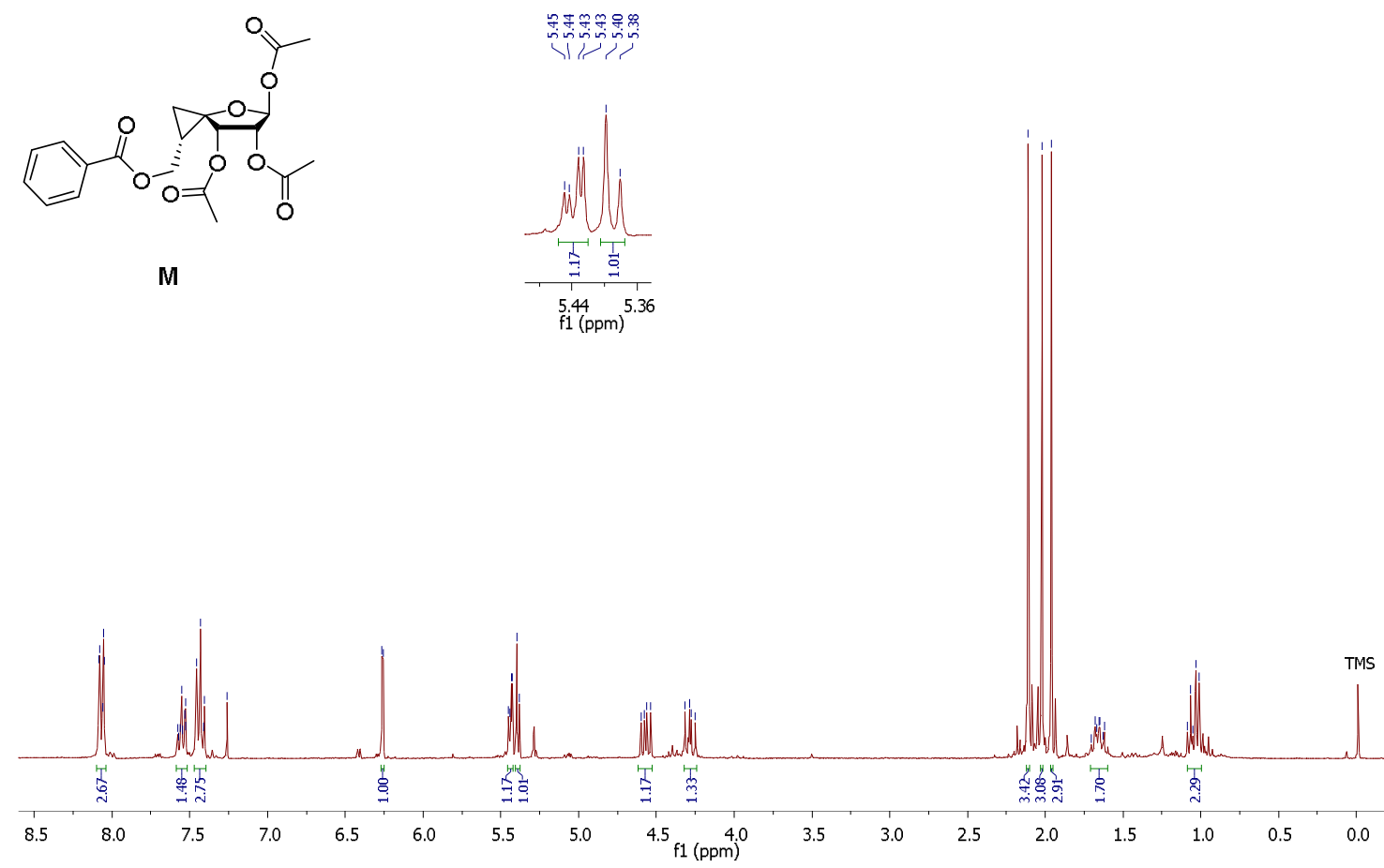

${ }^{13} \mathrm{C}\left\{{ }^{1} \mathrm{H}\right\} \mathrm{NMR}, 75 \mathrm{M} \mathrm{Hz}, \mathrm{CDCl}_{3}$ (contains $10 \%$ of the minor isomer)
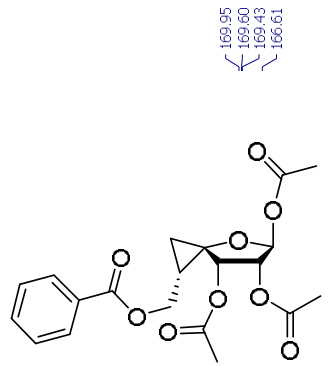

M

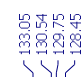

$\underset{\substack{0 \\ \infty \\ 0}}{i}$

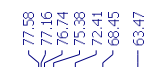

(1)

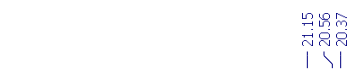

| ।

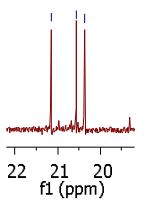

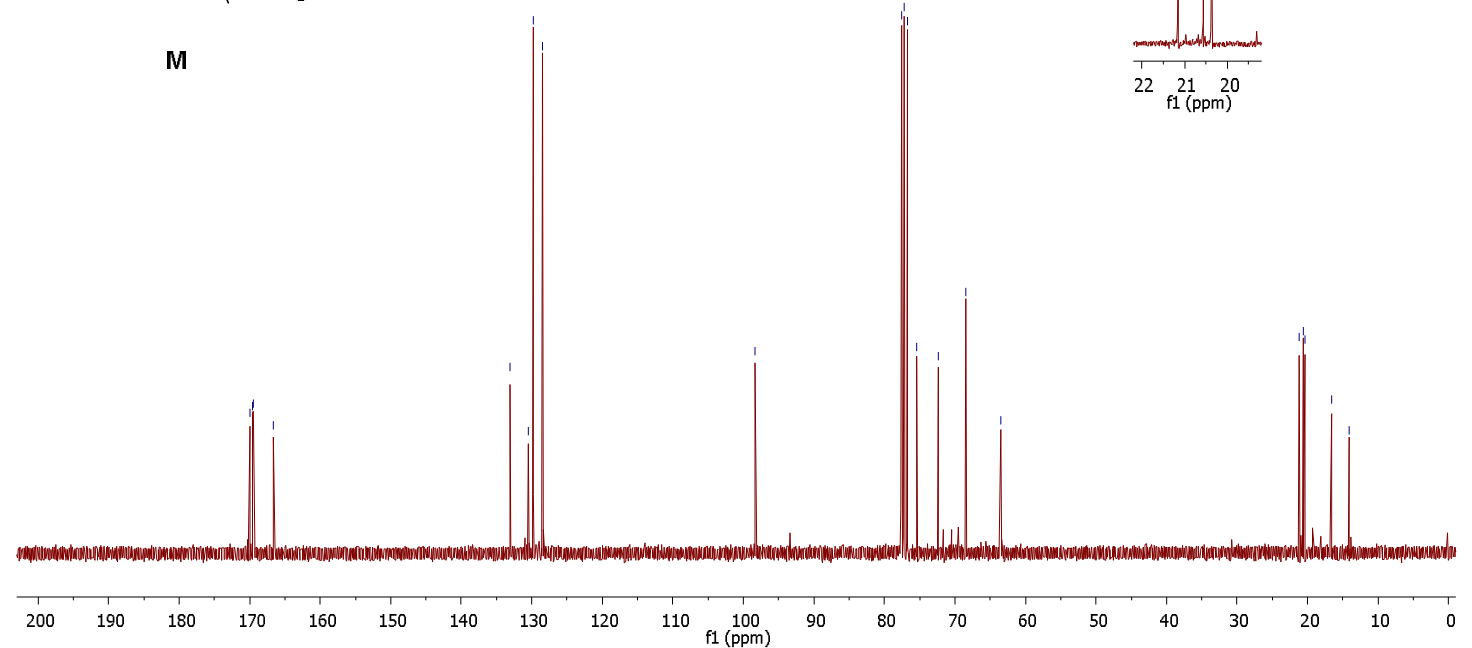


${ }^{1} \mathrm{H}$ NMR, 300 MHz, DMSO-d 6
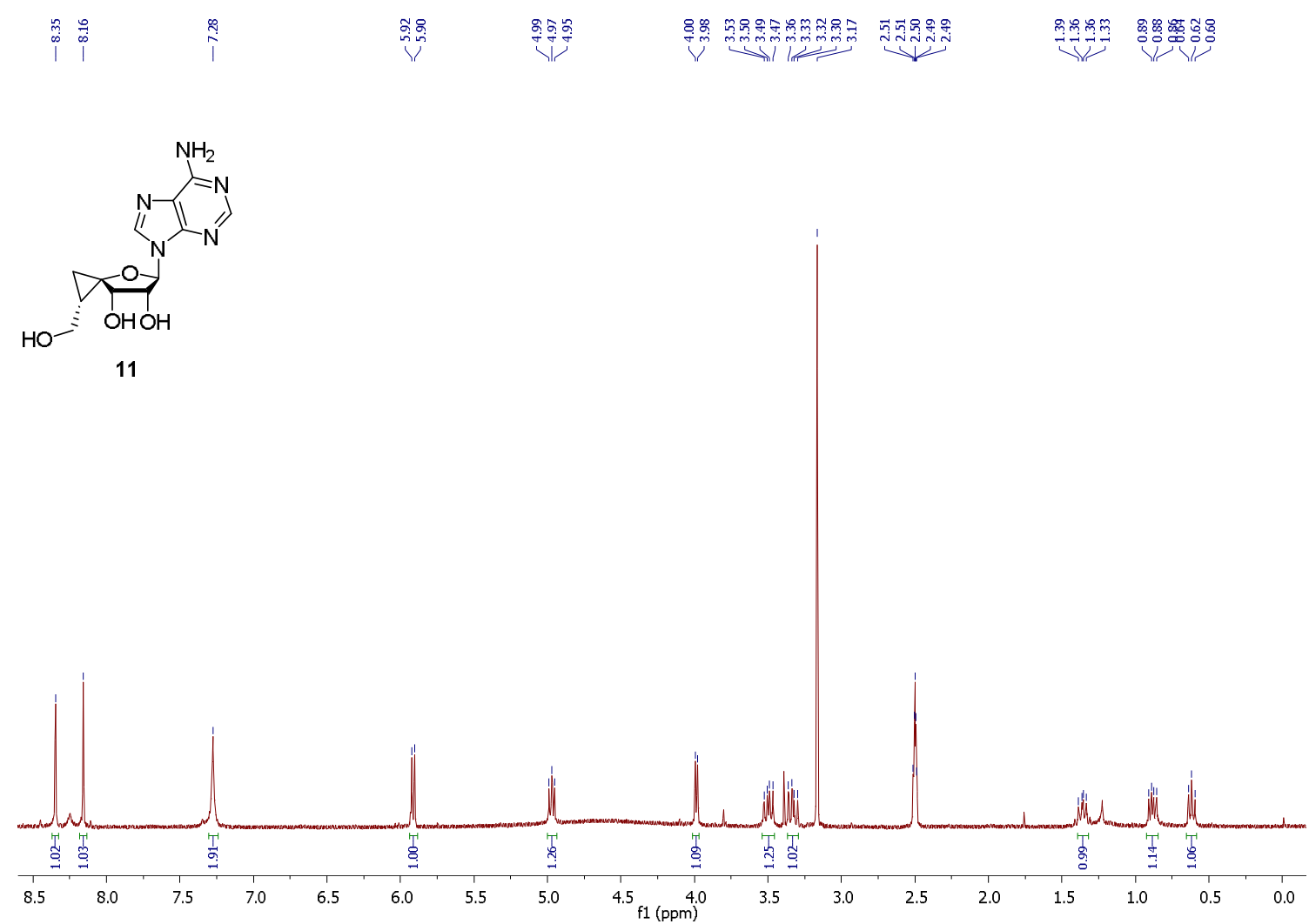

\section{${ }^{13} \mathrm{C}\left\{{ }^{1} \mathrm{H}\right\}$ NMR, $75 \mathrm{MHz}, \mathrm{DM} \mathrm{SO}-\mathrm{d}_{6}$}<smiles>Nc1ncnc2c1ncn2C(O)C1CC1CO</smiles>

11

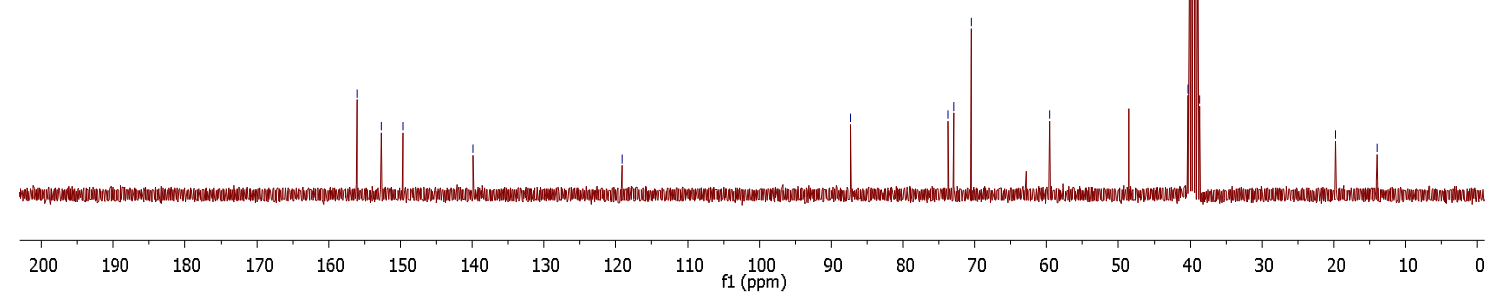


${ }^{1} \mathrm{H}$ NMR, $400 \mathrm{MHz}$, Methanol-d 4
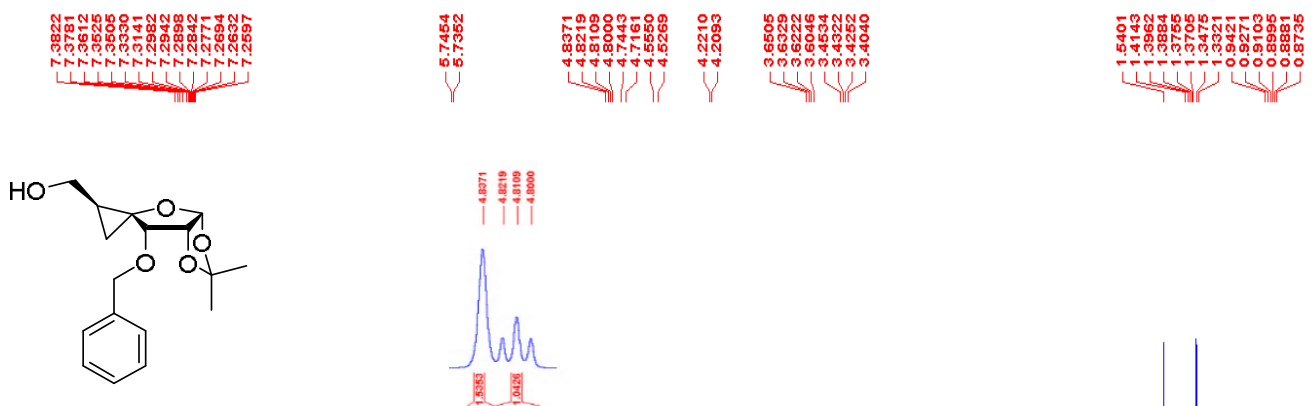

N
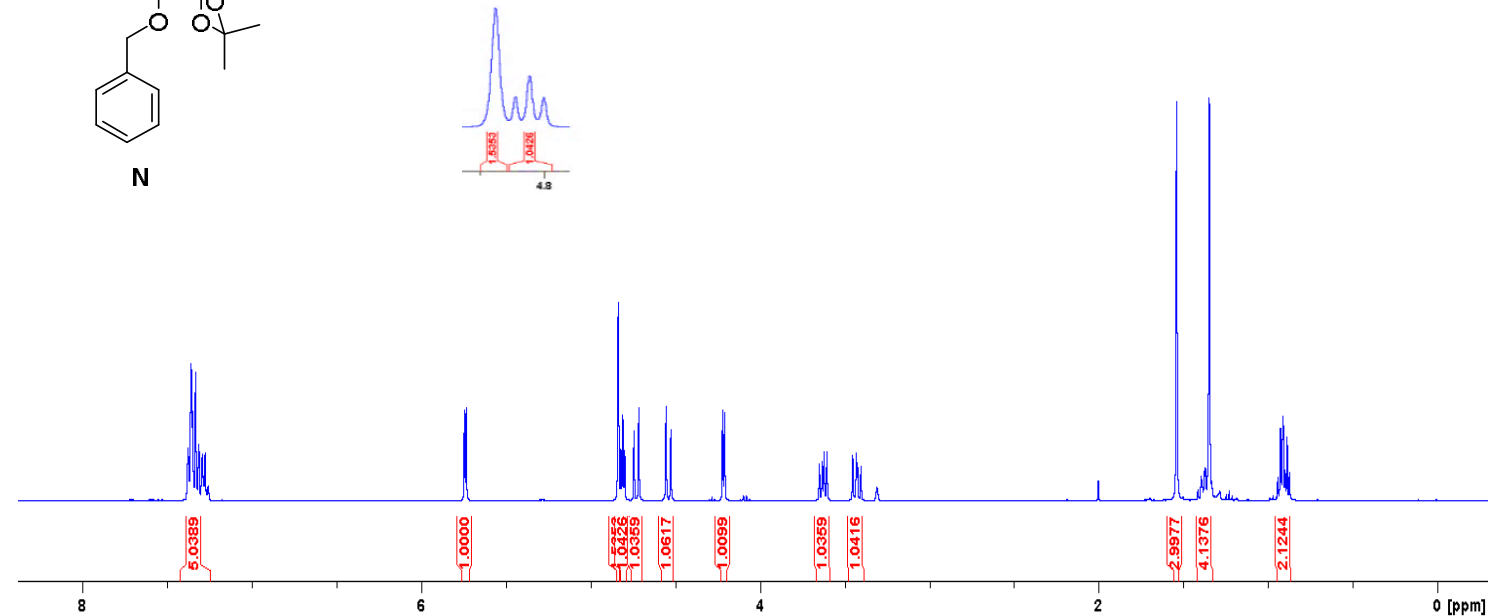

${ }^{13} \mathrm{C}\{\mathrm{H}\}$ NMR, $75 \mathrm{MHz}$, Methanol- $\mathrm{d}_{4}$

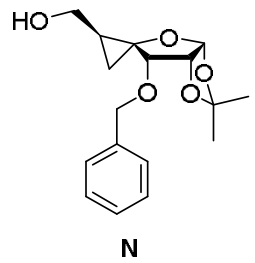

il
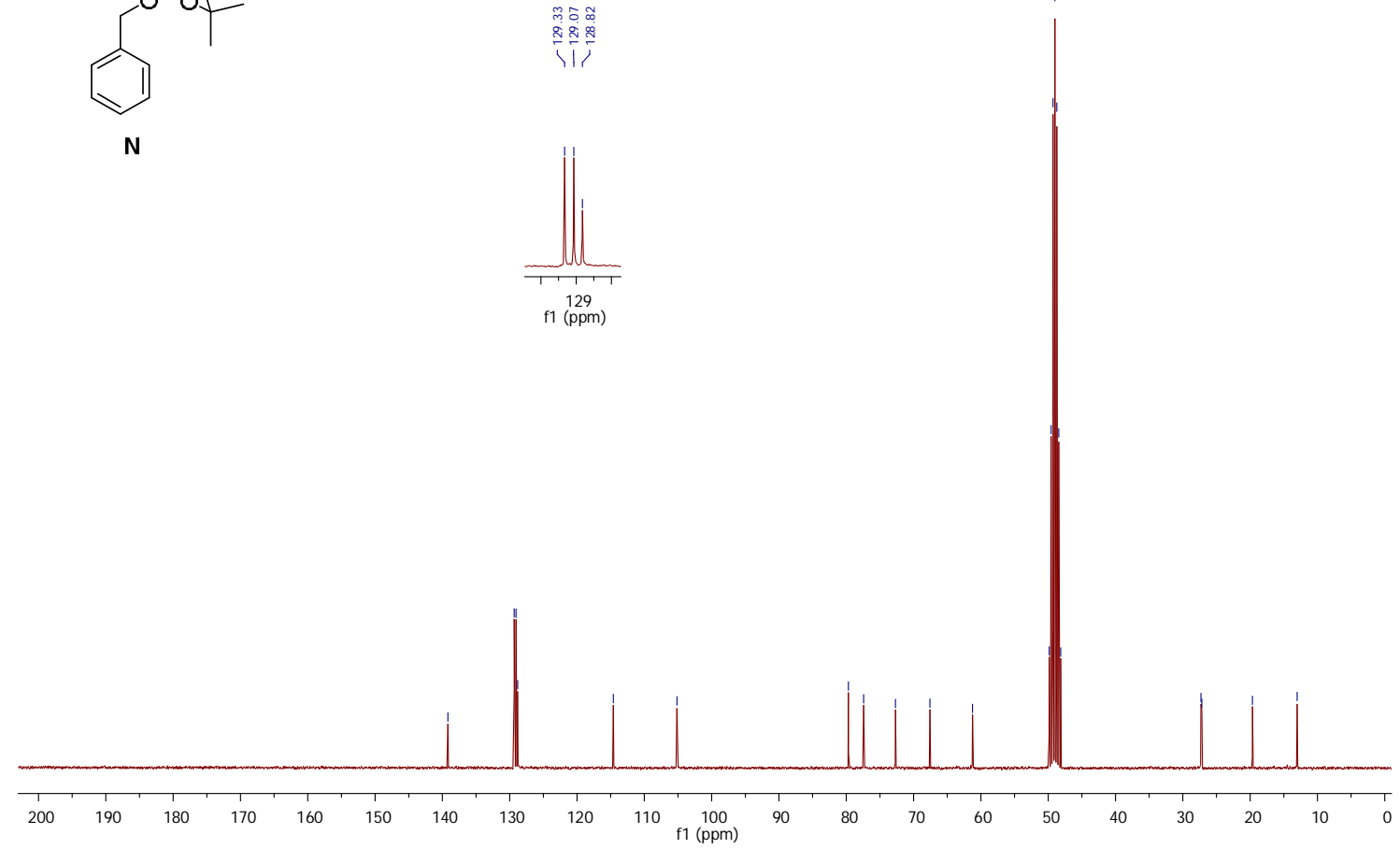
${ }^{1} \mathrm{H}$ NMR, $400 \mathrm{MHz}$, Methanol-d

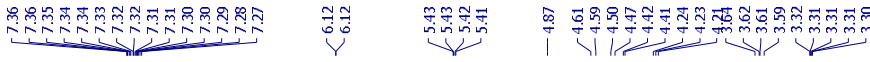

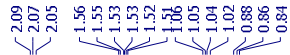

(1)

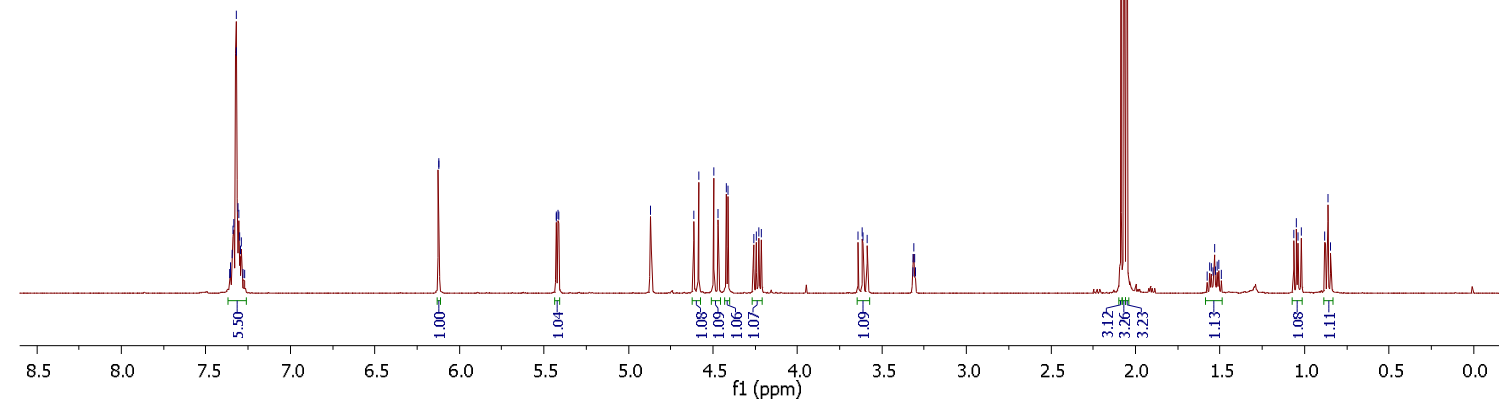

${ }^{13} \mathrm{C}\left\{{ }^{\mathrm{H}} \mathrm{H}\right\} \mathrm{NMR}, 75 \mathrm{M} \mathrm{Hz}$, Methanol-d

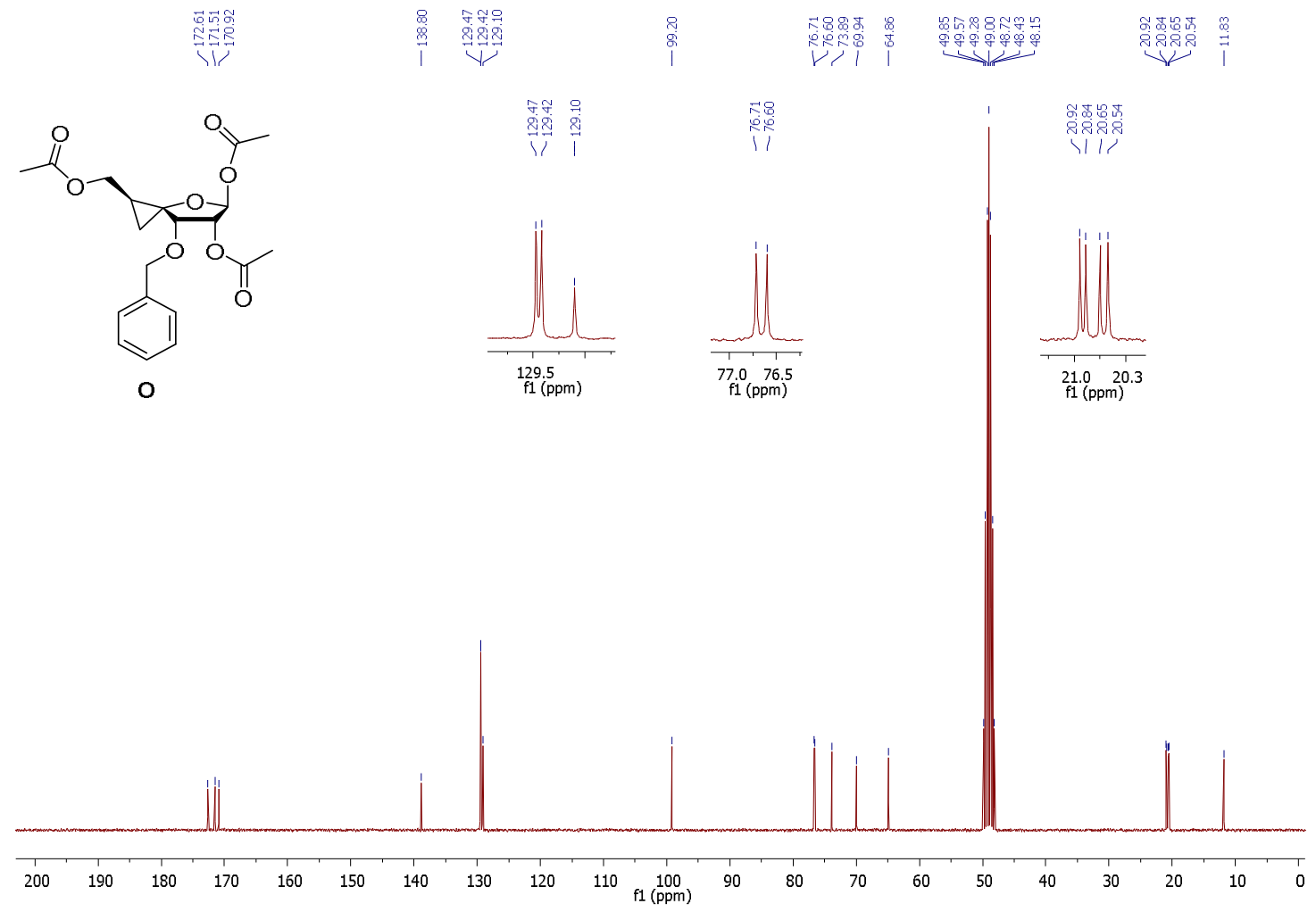


${ }^{1} \mathrm{H}$ NMR, 300 MHz, DMSO-d 6

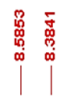
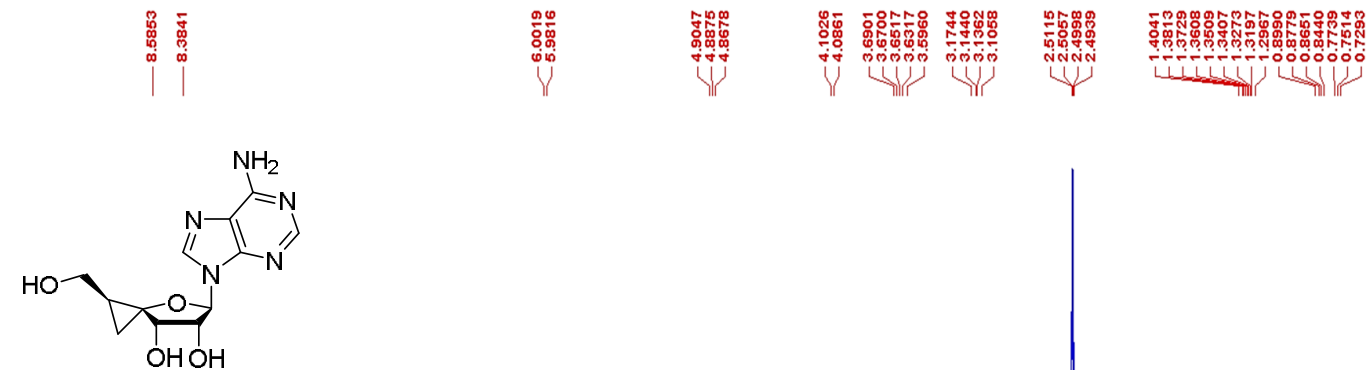

12

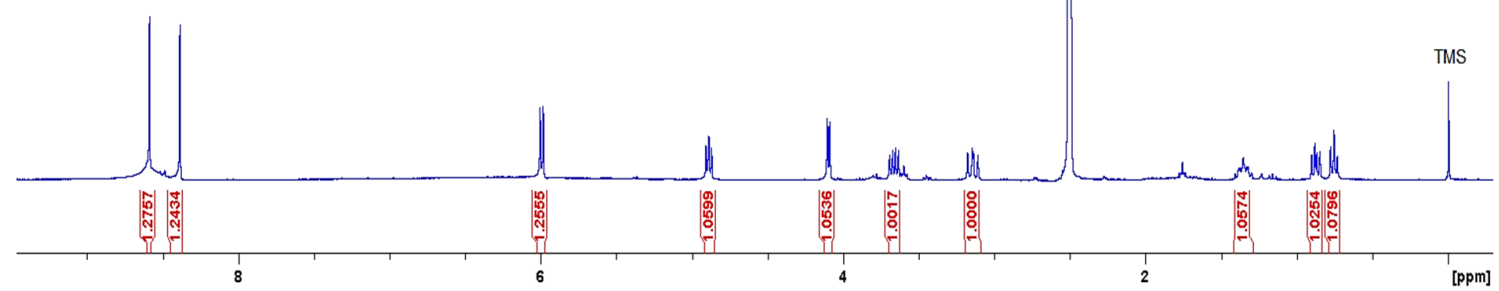

${ }^{13} \mathrm{C}\left\{{ }^{\mathrm{H}} \mathrm{H}\right\}$ NMR, $75 \mathrm{MHz}$, DM SO-d $\mathrm{d}_{6}$
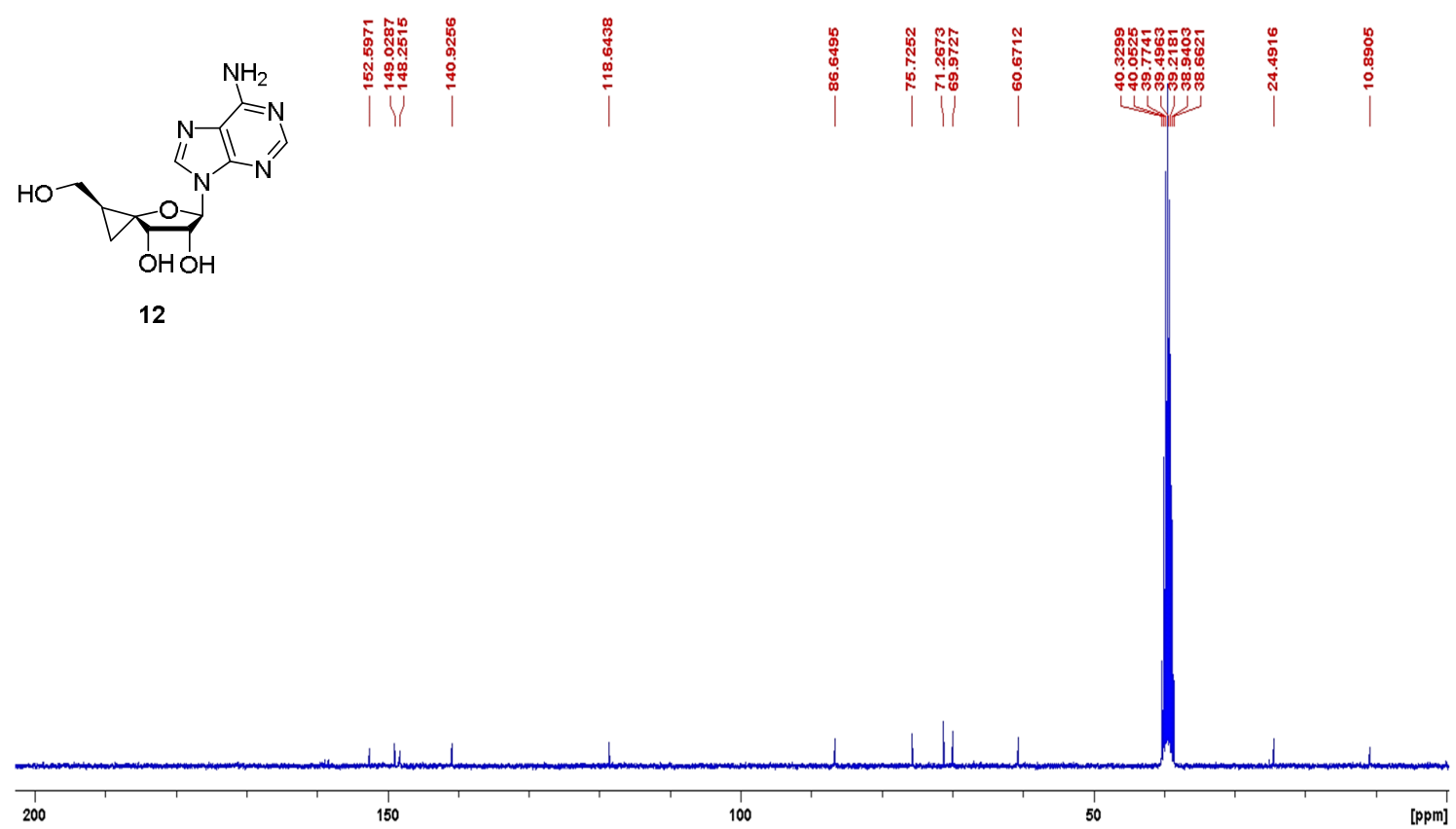
${ }^{1} \mathrm{H}$ NM R, $300 \mathrm{MHz}$, Methanol-d $\mathrm{d}_{4}$ (contains traces of EtOAC [*])

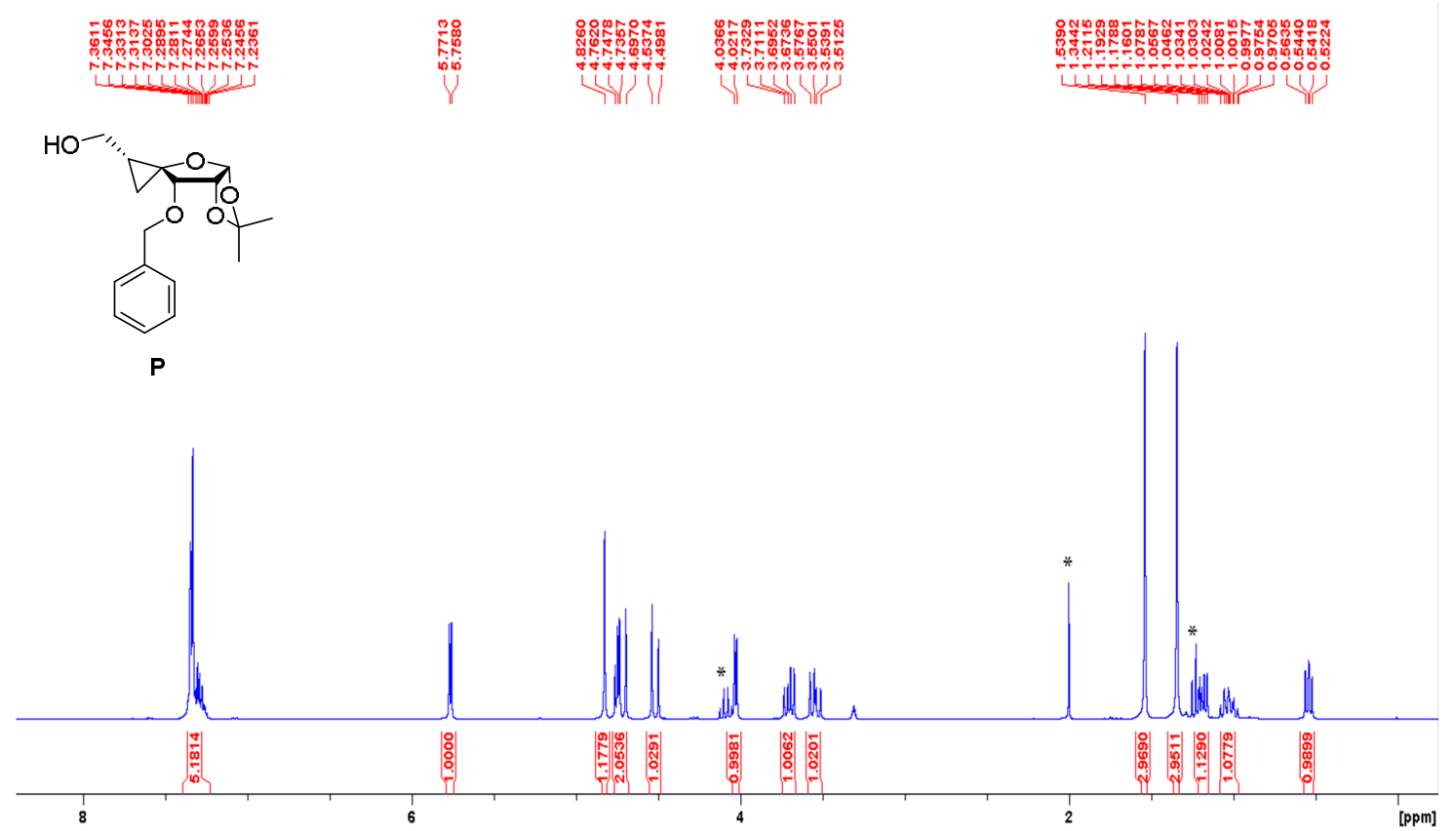

\section{$\left.{ }^{13} C_{\{}{ }^{1} H\right\} N M R, 75$ M Hz, Methanol- $d_{4}$}
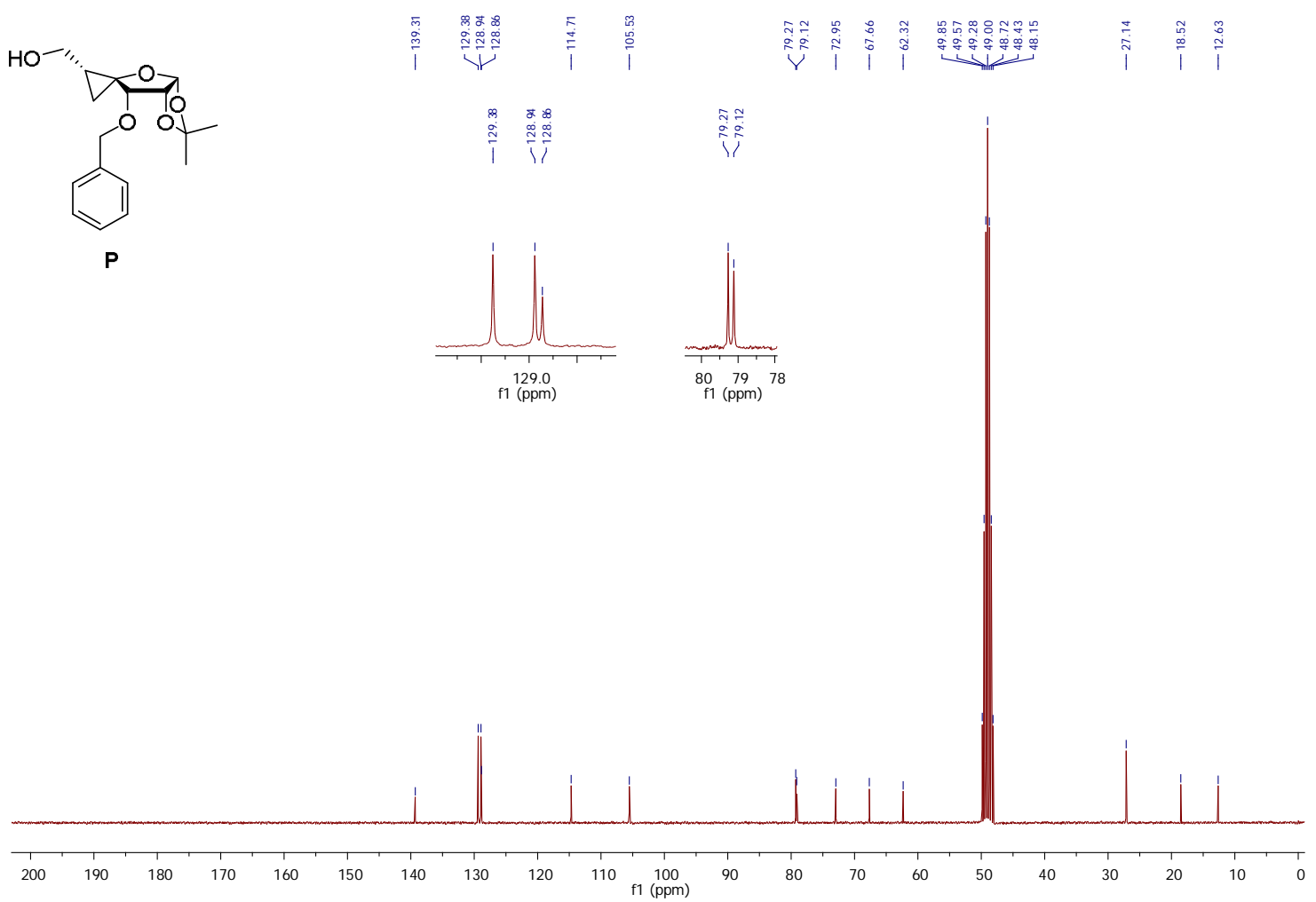
${ }^{1} \mathrm{H}$ NMR, $400 \mathrm{MHz}$, Methanol-d

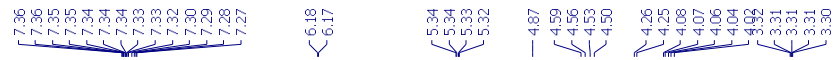

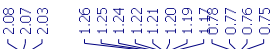<smiles>CC(=O)OC[C@@H]1CC12OC(OC(C)=O)[C@H](OC(C)=O)C2Oc1ccccc1</smiles>

Q

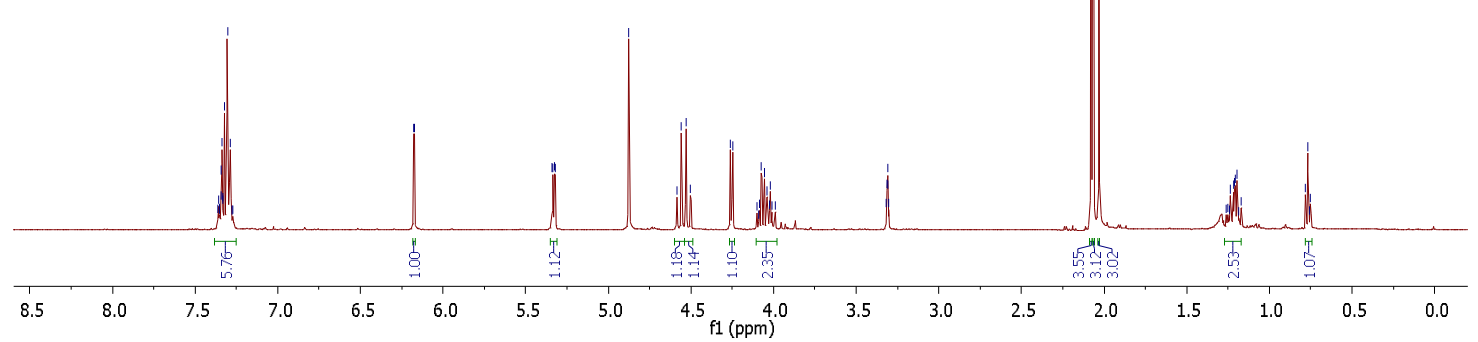

${ }^{13} \mathrm{C}\left\{{ }^{\mathrm{l}} \mathrm{H}\right\}$ NMR, $75 \mathrm{M} \mathrm{Hz}$, Methanol- $\mathrm{d}_{4}$

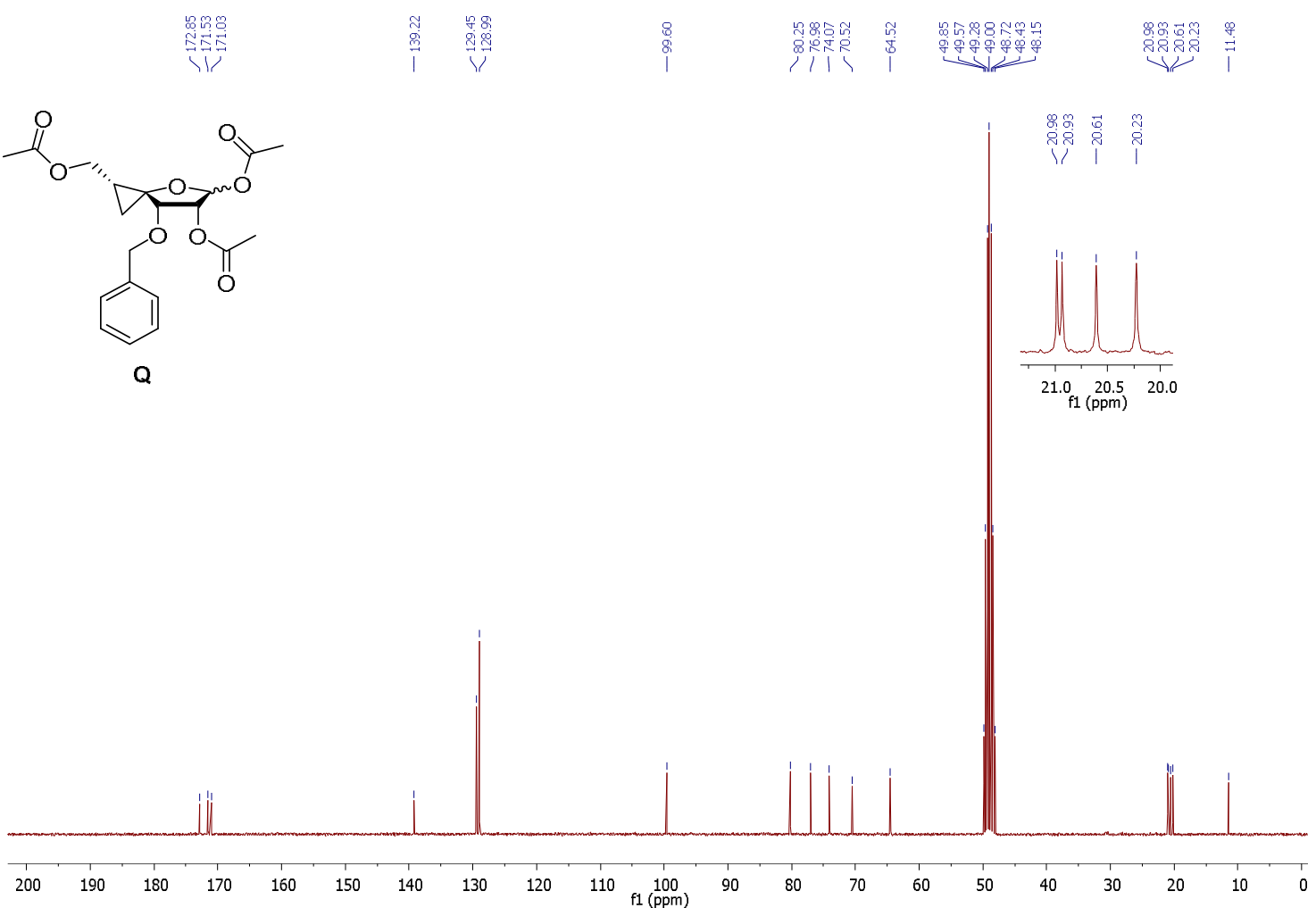

S-50 
${ }^{1} \mathrm{H}$ NMR, 300 MHz, Methanol-d 4

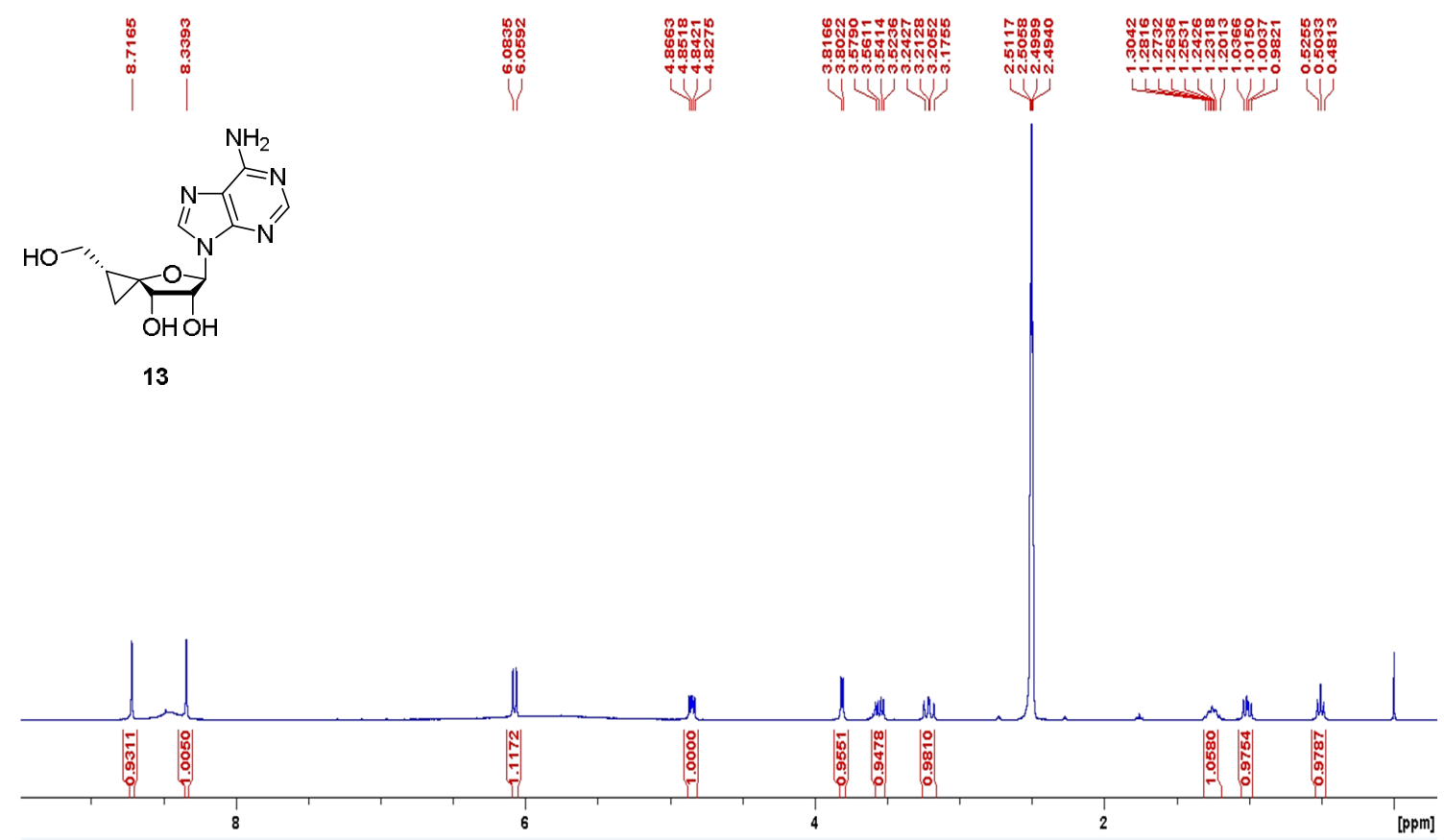

${ }^{13} \mathrm{C}\left\{{ }^{1} \mathrm{H}\right\}$ NMR, $75 \mathrm{M} \mathrm{Hz}$, Methanol-d 4

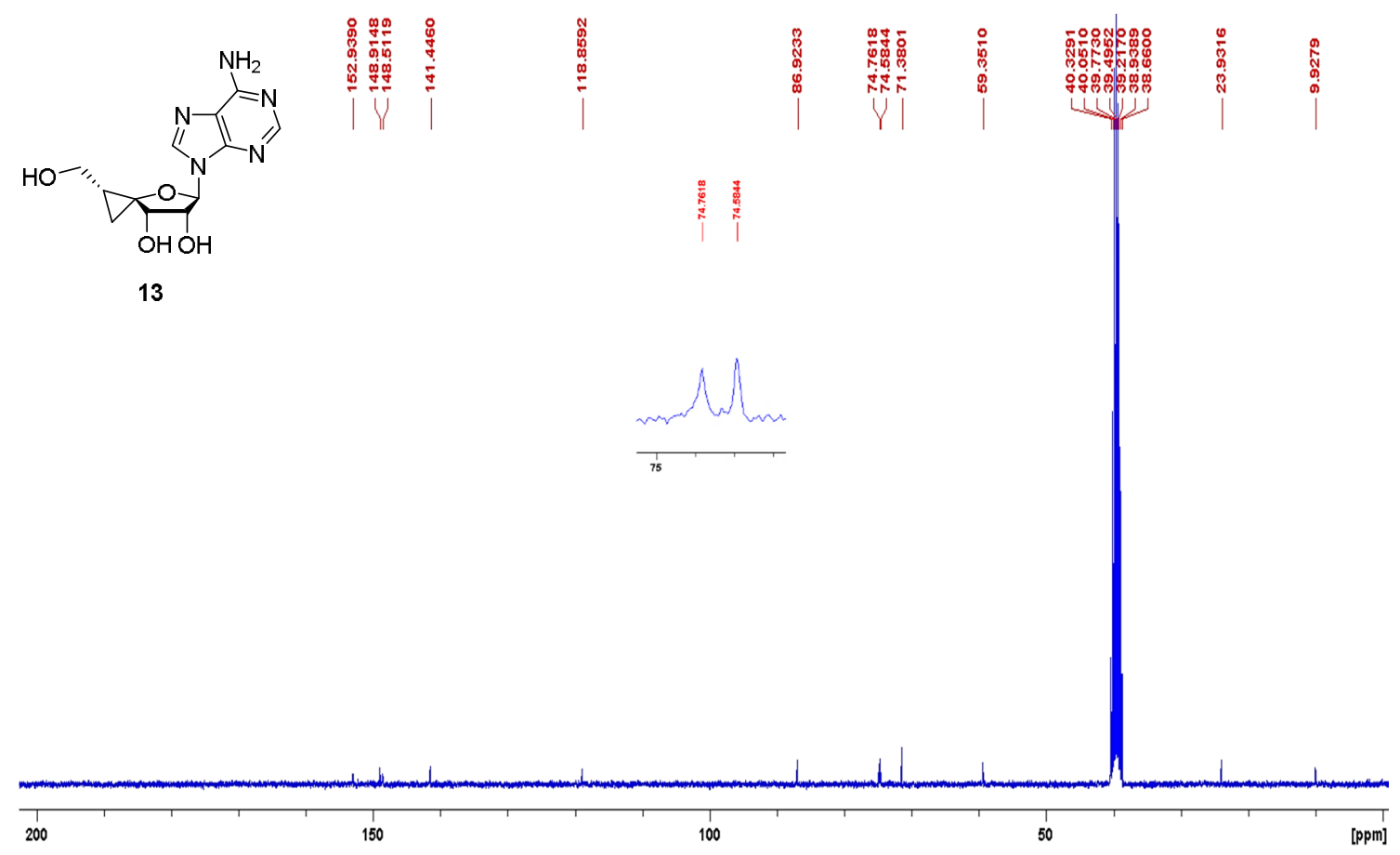




\section{Stereochemistry determination by 2D NMR spectroscopy}

* Compound $\alpha^{\prime}$-cis-6:

\section{COSY NMR, $600 \mathrm{MHz}^{\mathrm{CDCl}}{ }_{3}$}
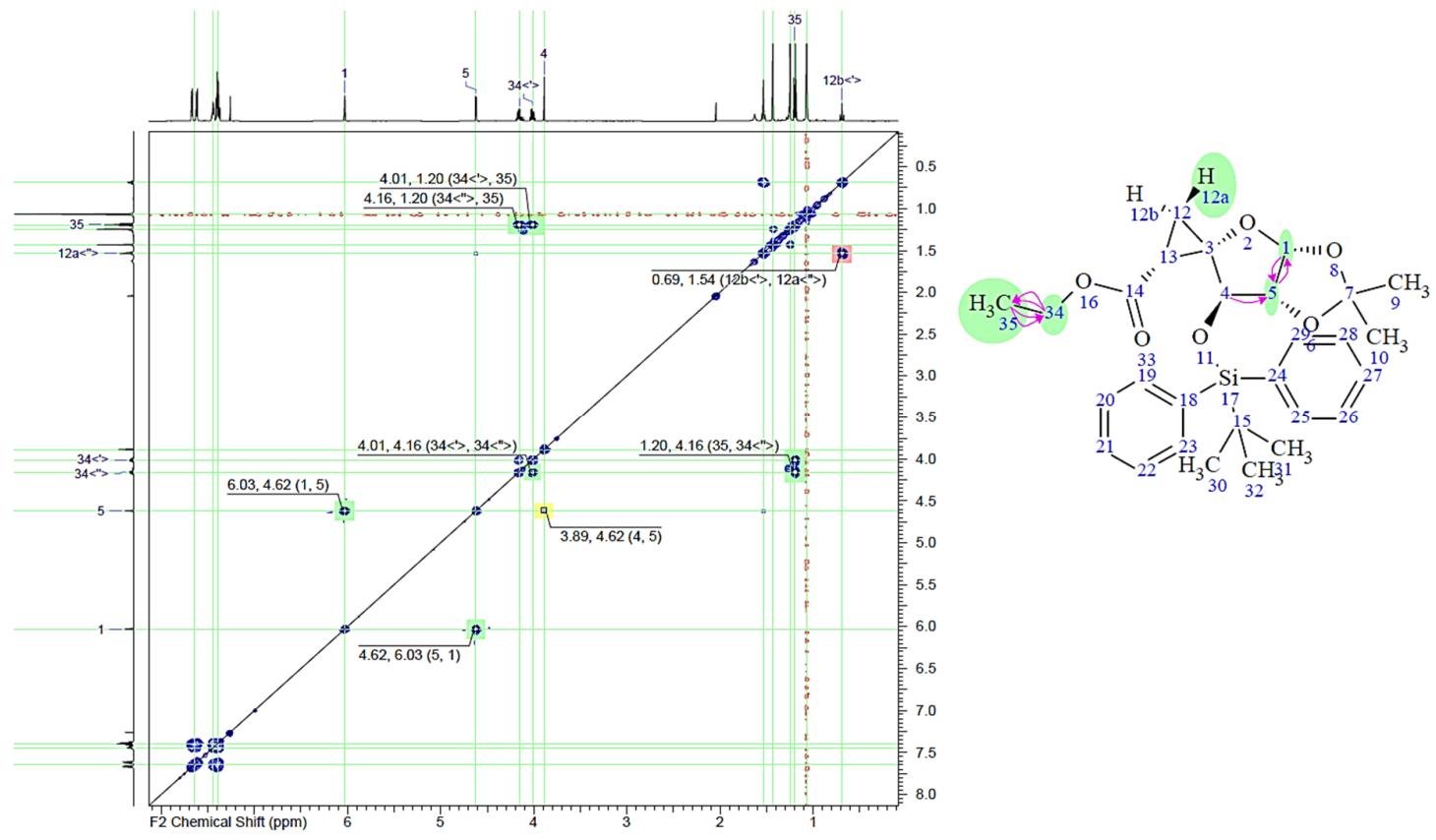

NOESY NMR, $600 \mathrm{MHz}, \mathrm{CDCl}_{3}$

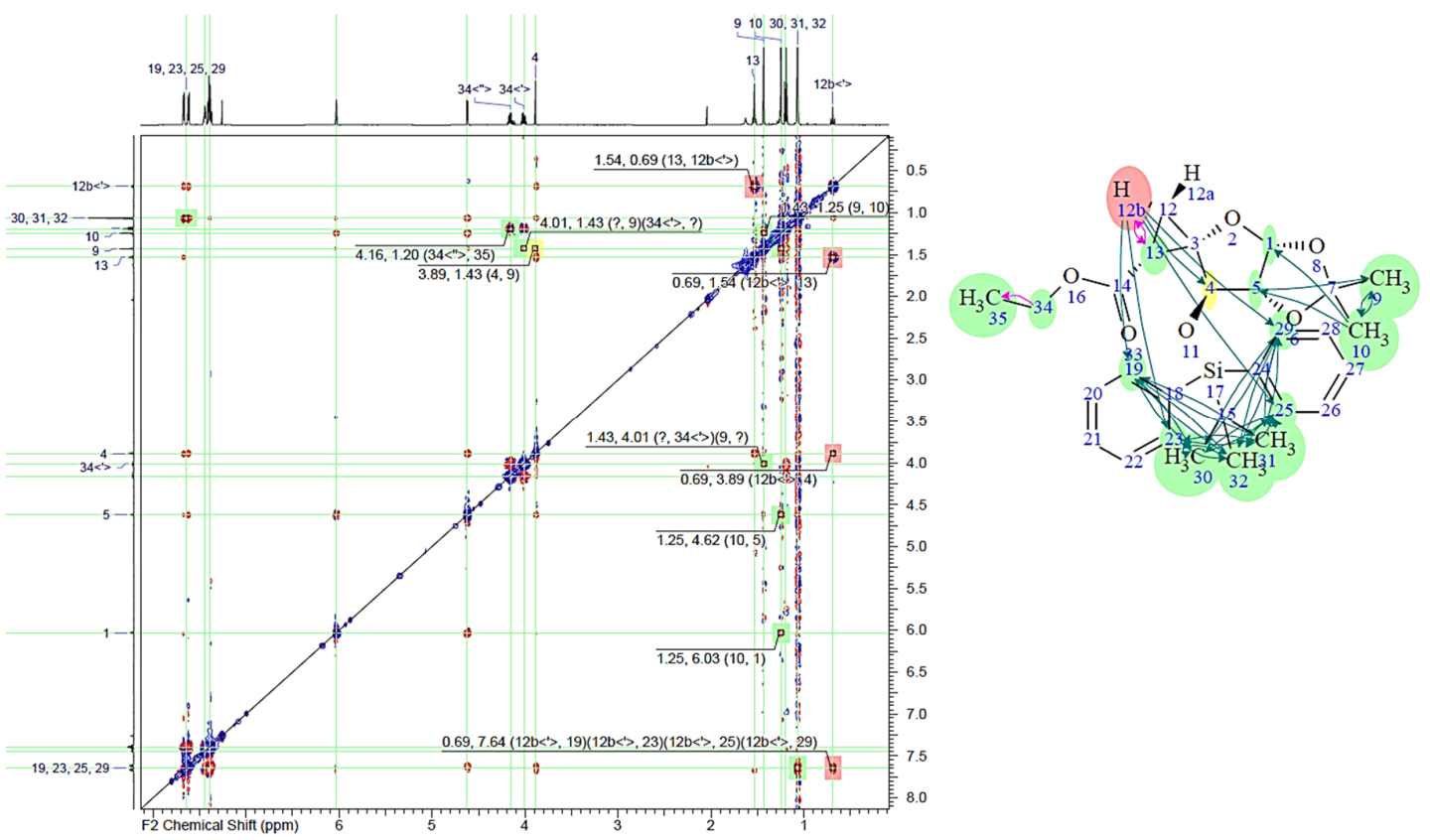


HSQC-DEPT NMR, $150 \mathrm{MHz}, \mathrm{CDCl}_{3}$

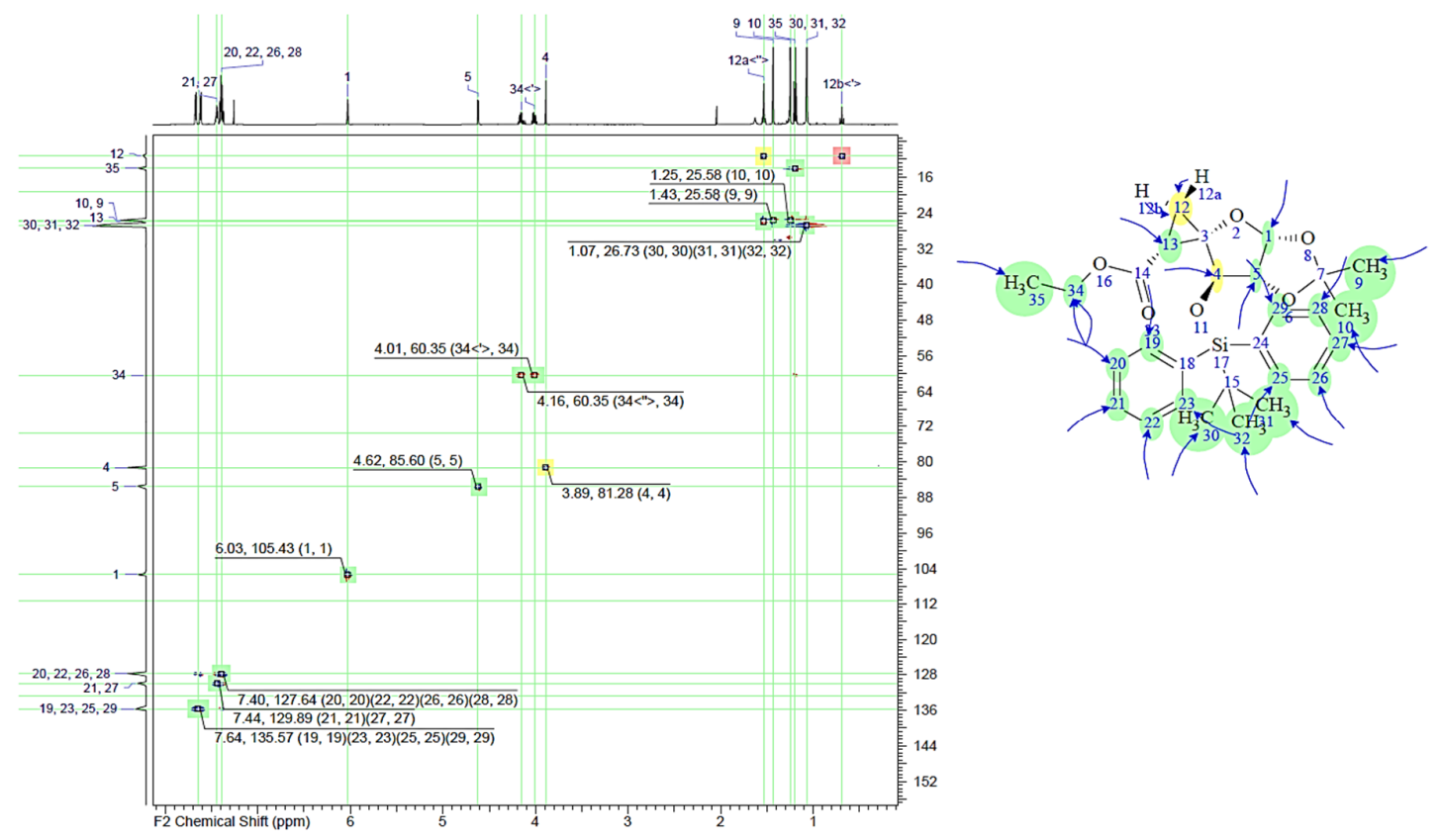

HBMC NMR, $150 \mathrm{MHz}, \mathrm{CDCl}_{3}$
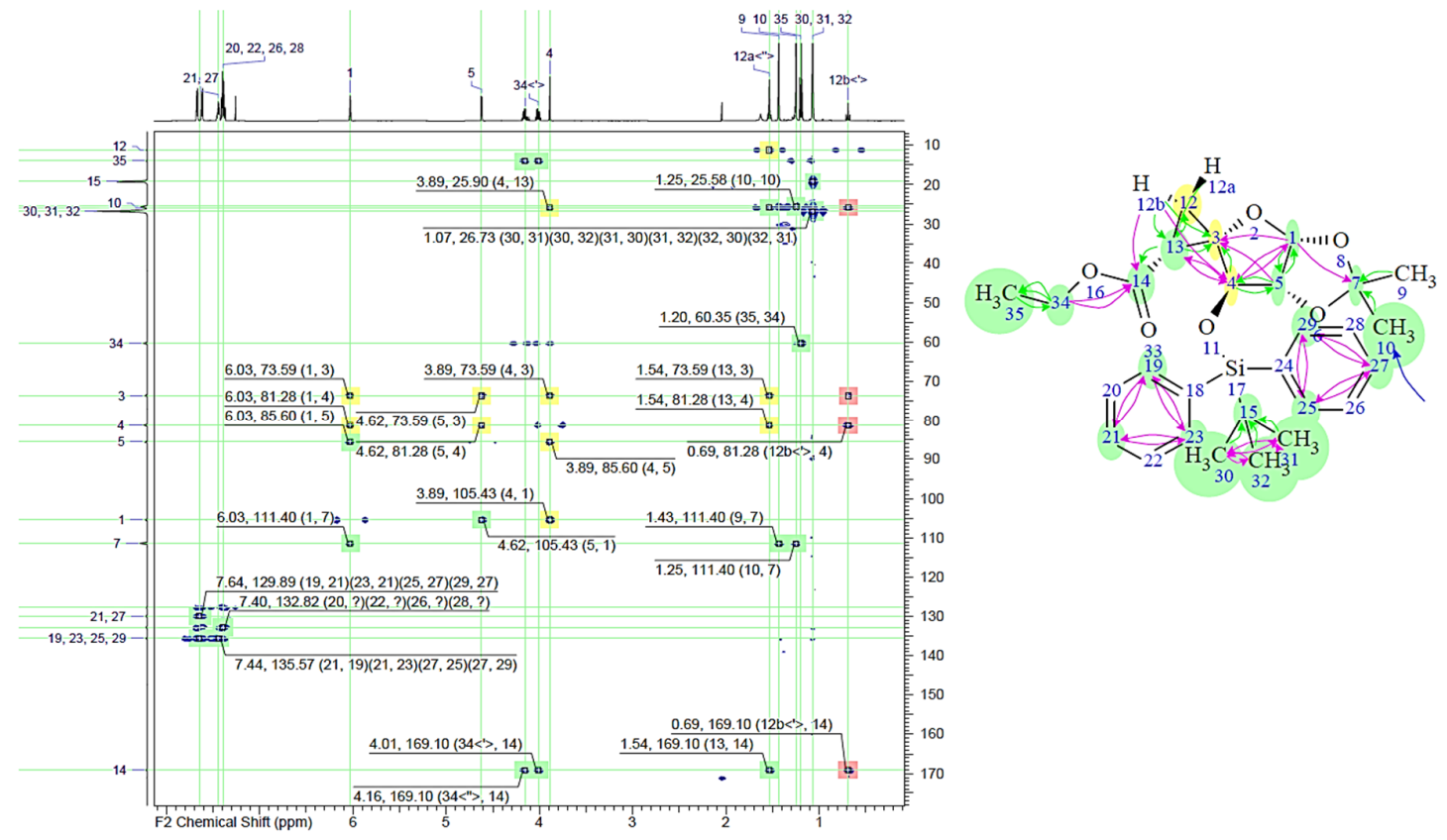


\section{* Compound $\alpha^{\prime}$-trans-6.}

\section{COSY NM R, $400 \mathrm{MHz}, \mathrm{CDCl}_{3}$}

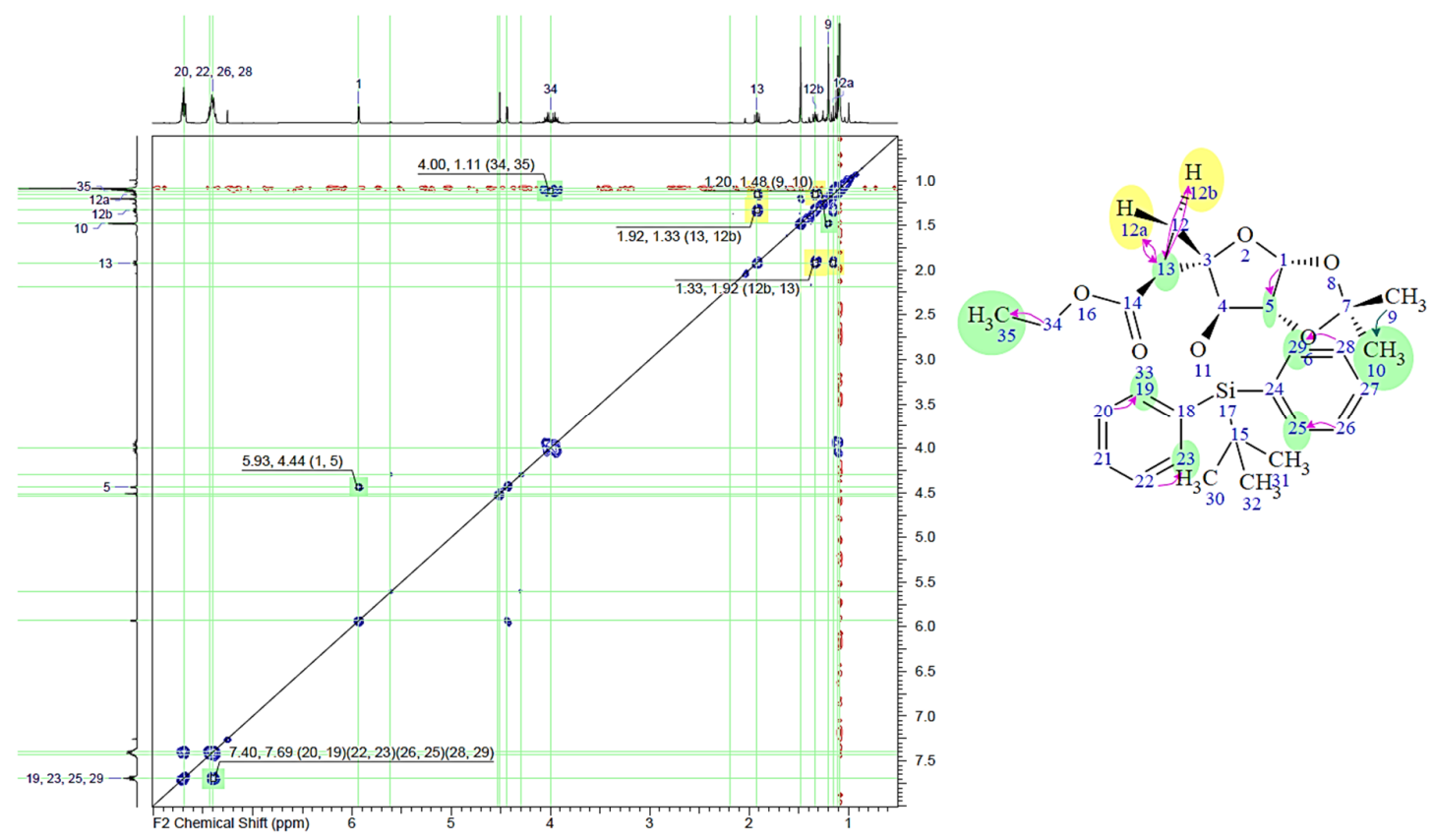

\section{NOESY NMR, $400 \mathrm{M} \mathrm{Hz}, \mathrm{CDCl}_{3}$}

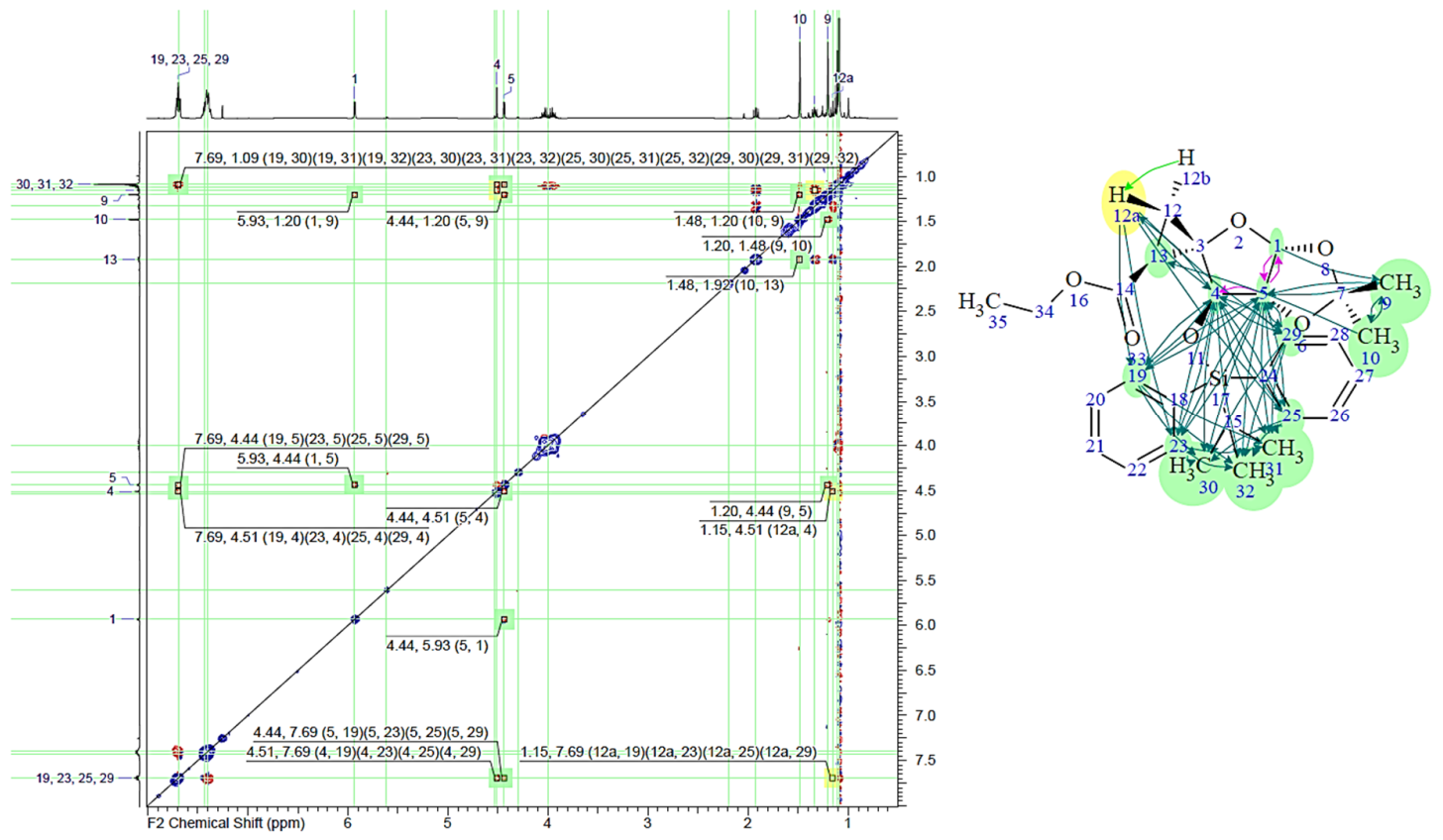


HSQC-DEPT NMR, $100 \mathrm{MHz}, \mathrm{CDC}_{3}$

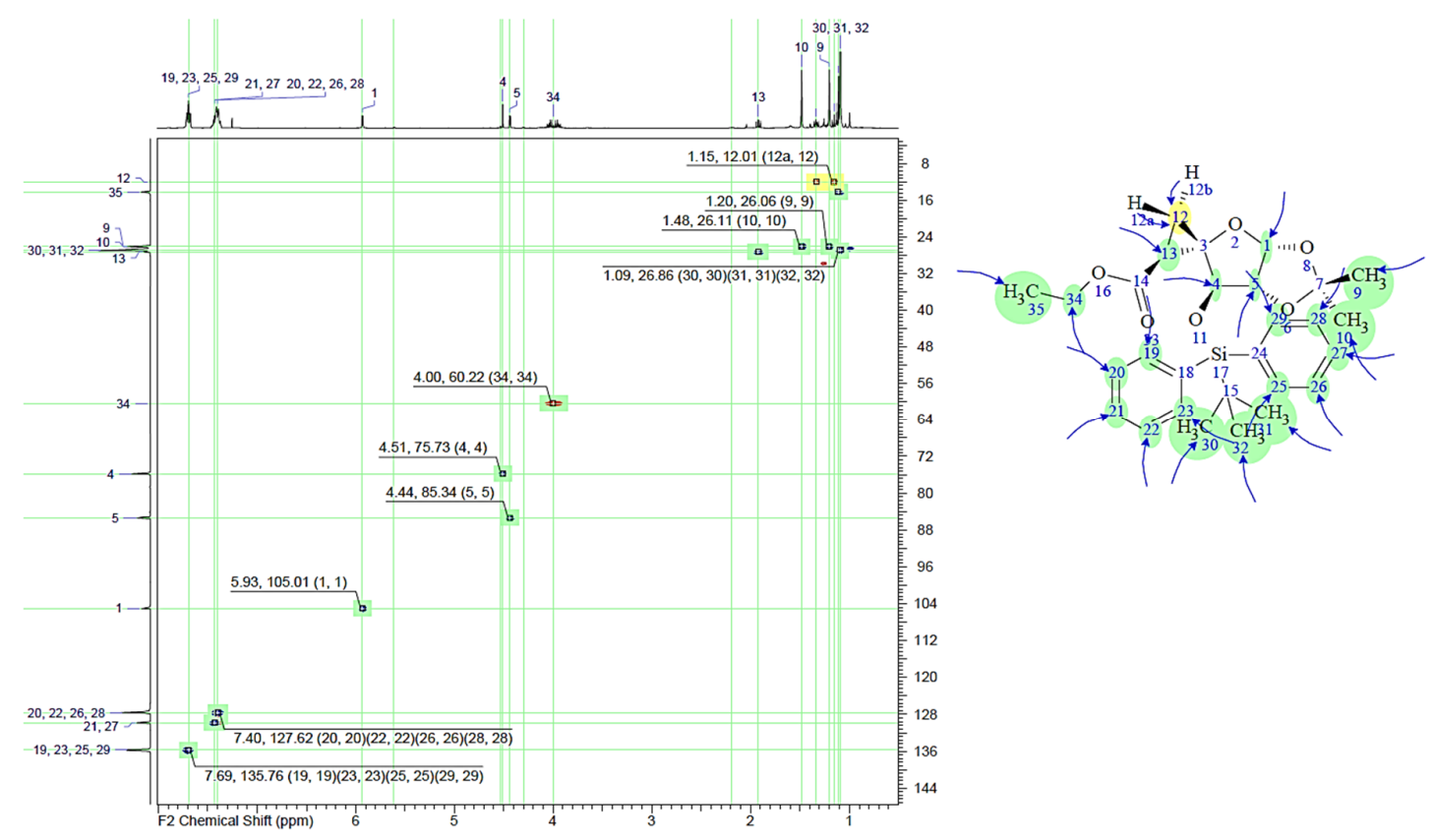

- Compound $\beta^{\prime}$-trans-9:

COSY NMR, 400 MHz, DMSO-d 6
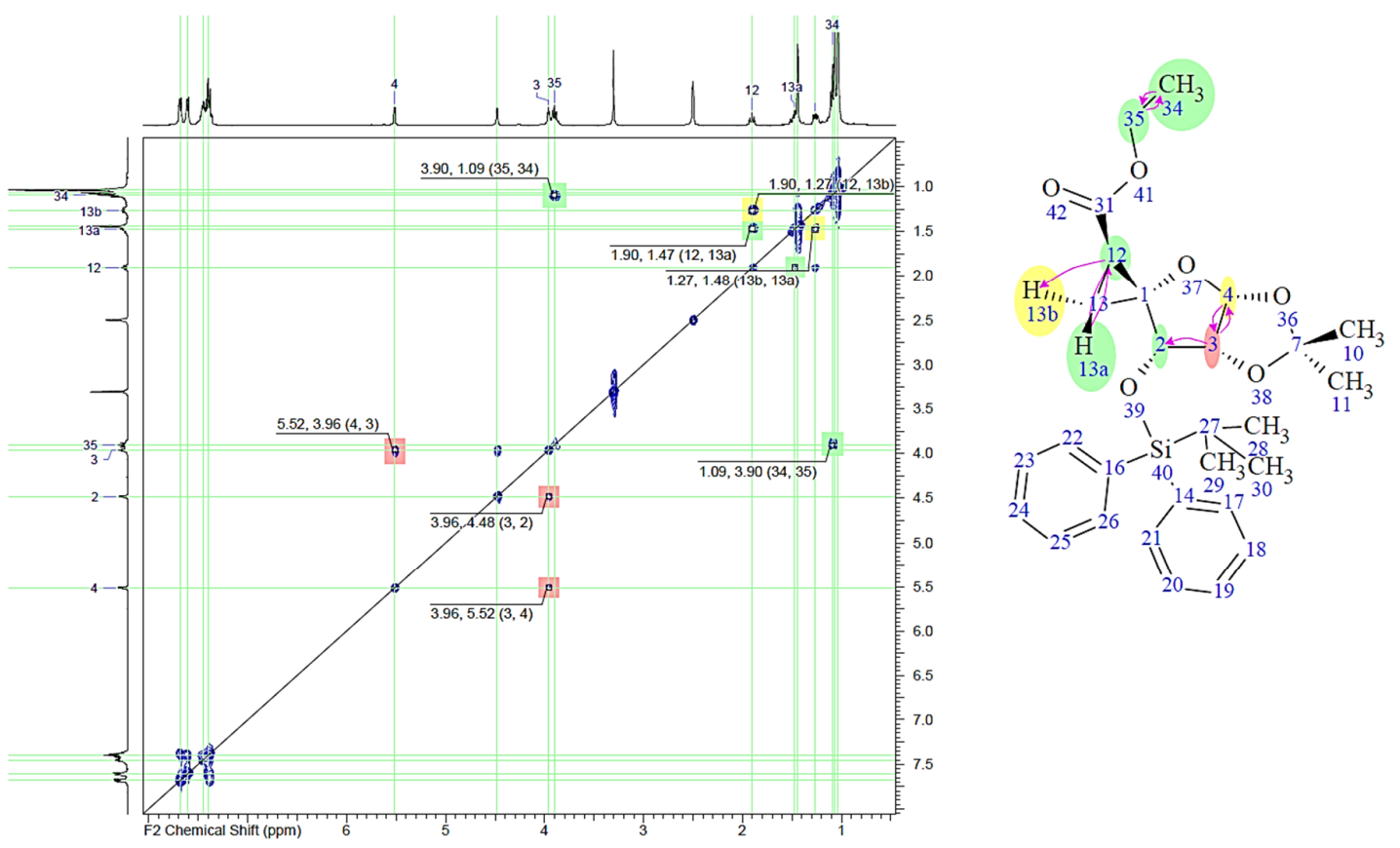

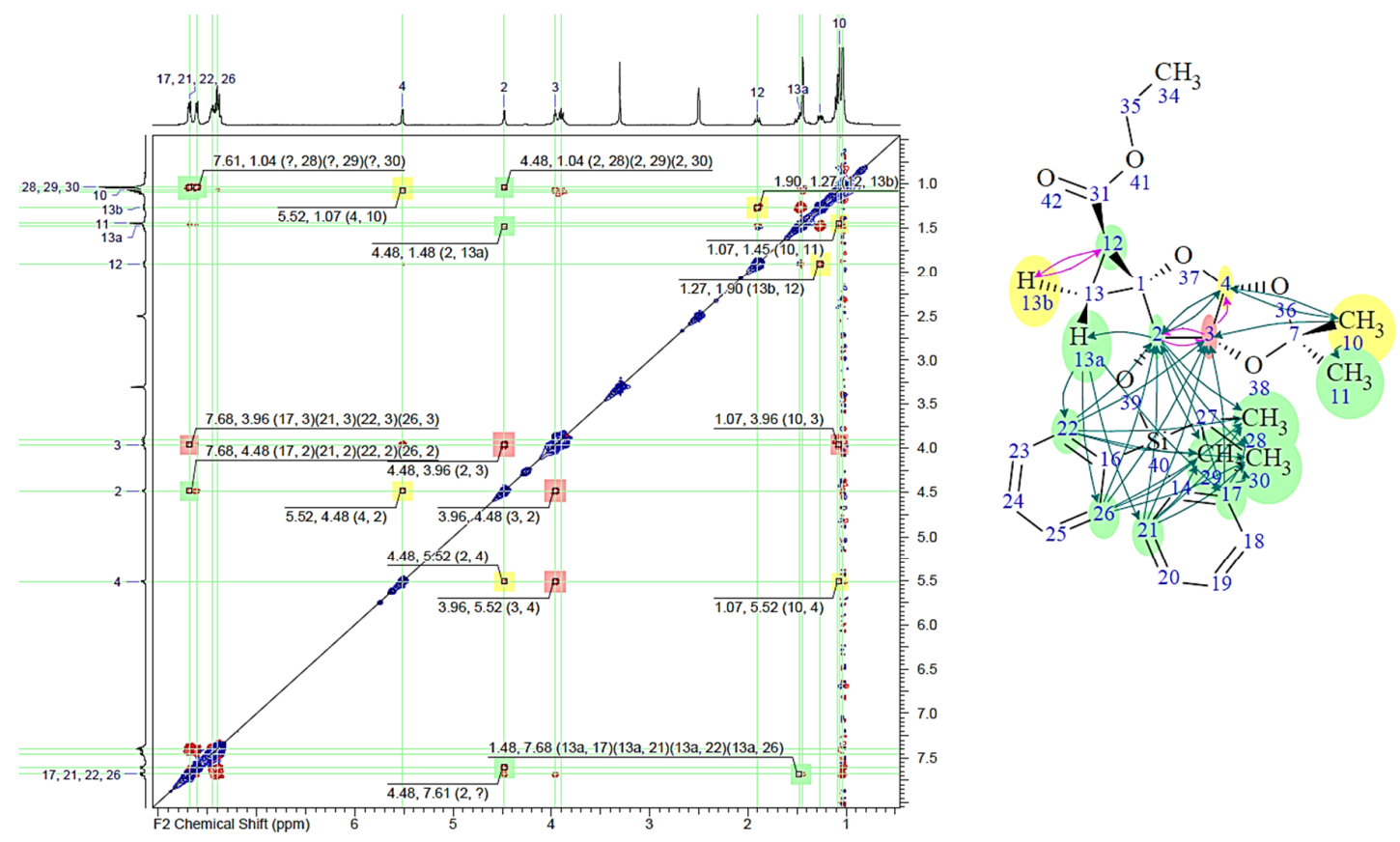

HSQC-DEPT NMR, 100 MHz, DMSO-d
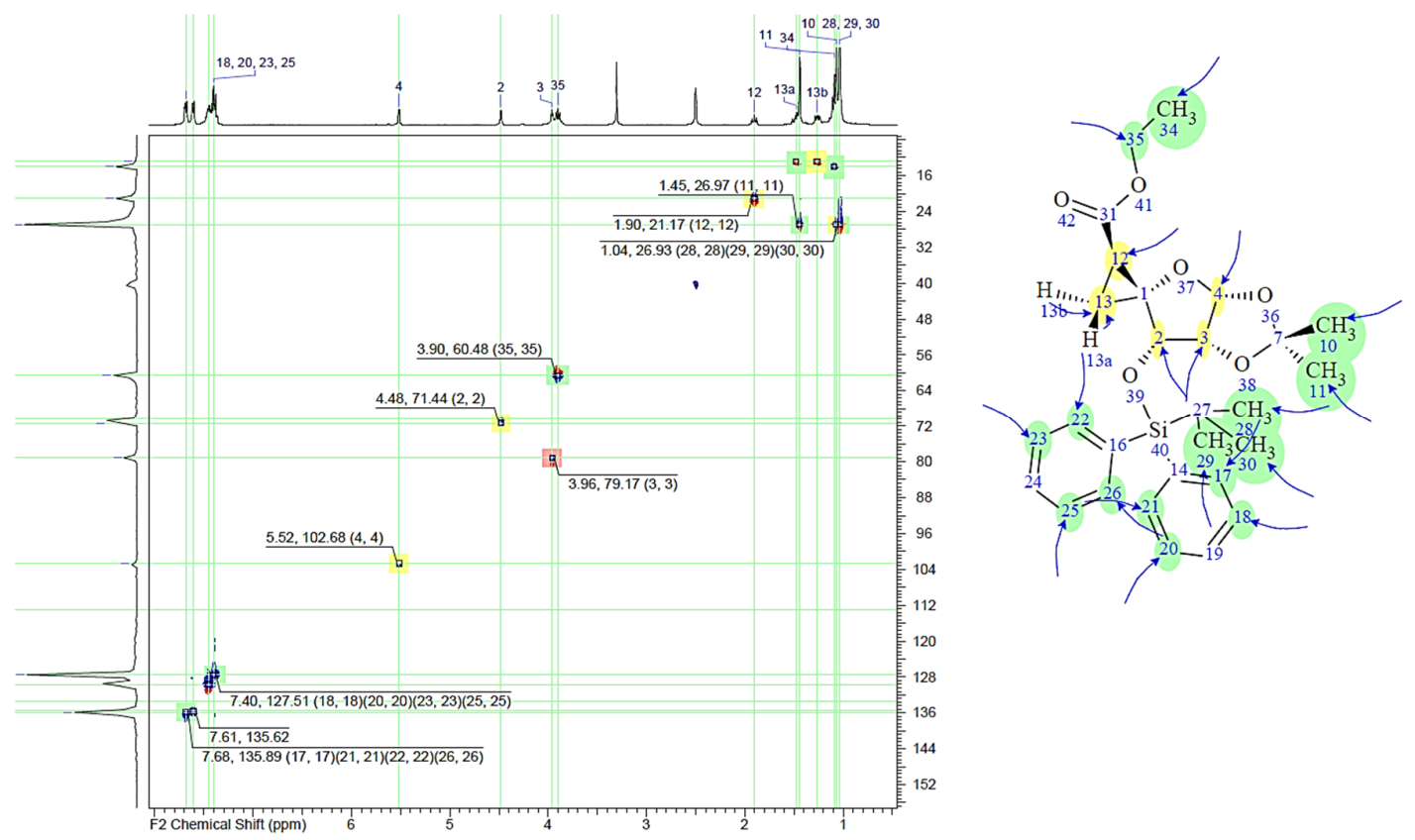
HMBC NMR, 100 MHz, DM SO-d 6
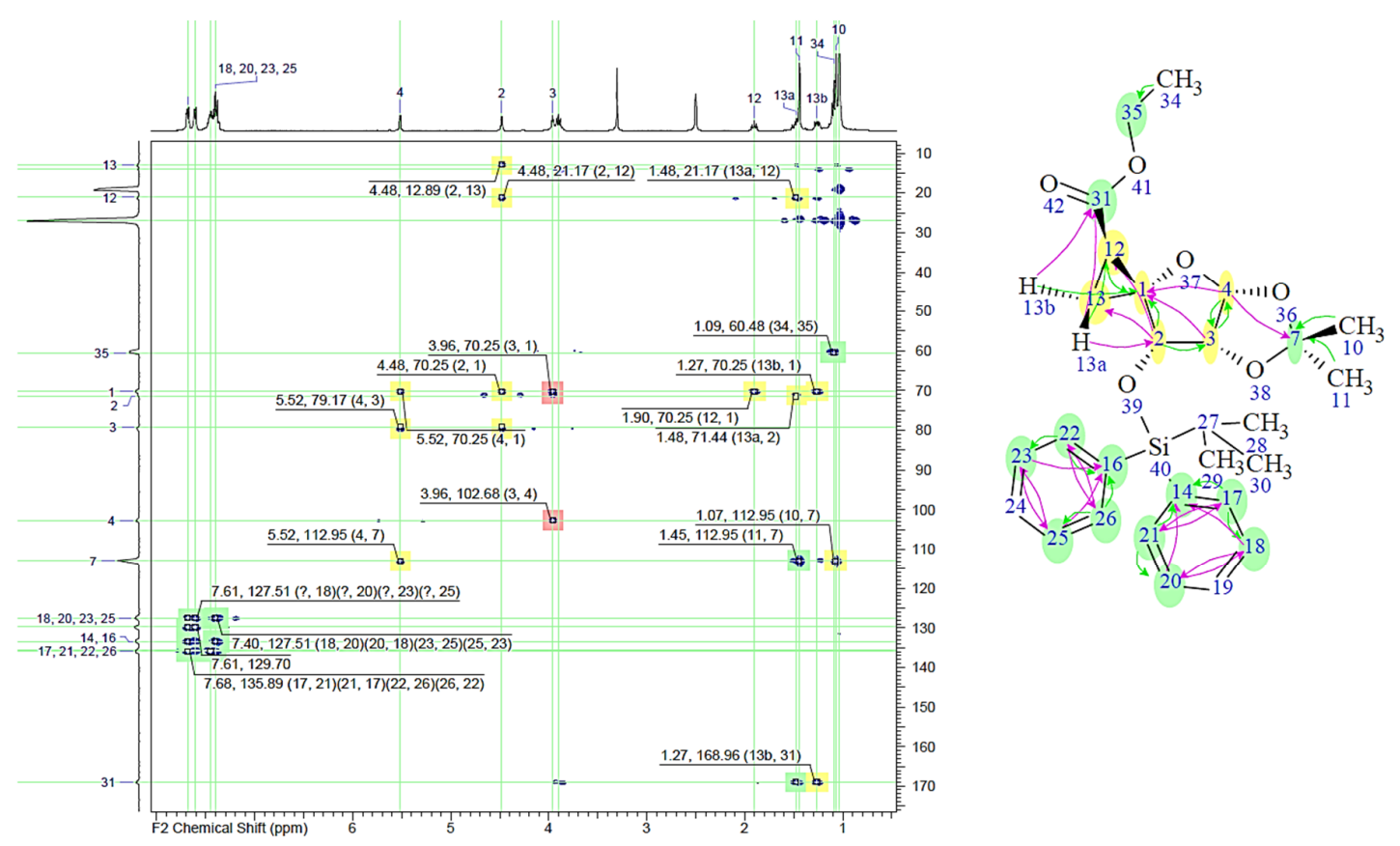

\section{* Compound $\beta^{\prime}$-cis-9:}

\section{NOESY NMR, $600 \mathrm{MHz}, \mathrm{C}_{6} \mathrm{D}_{6}$}

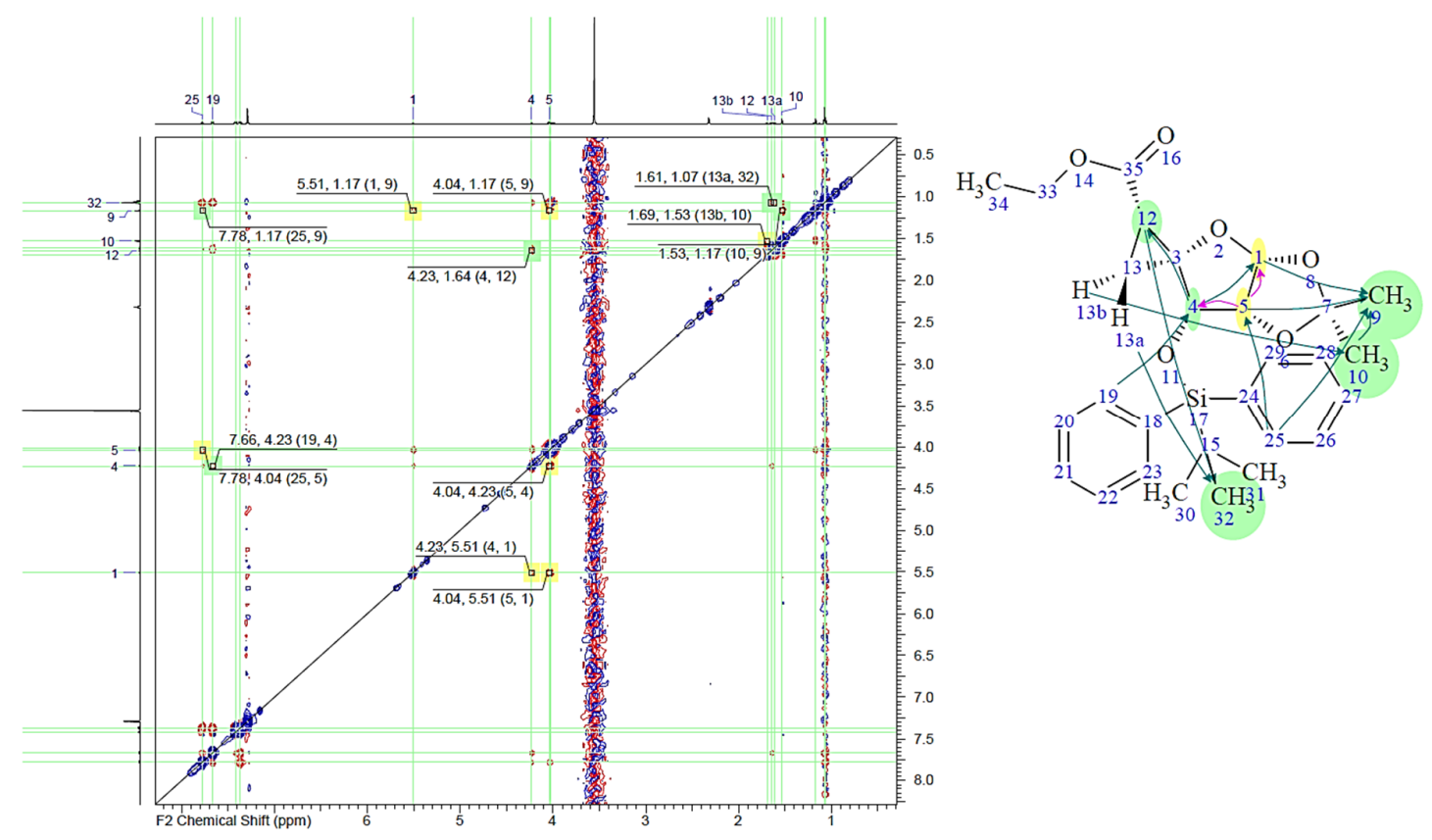


HSQC-DEPT NMR, $150 \mathrm{MHz}, \mathrm{C}_{6} \mathrm{D}_{6}$

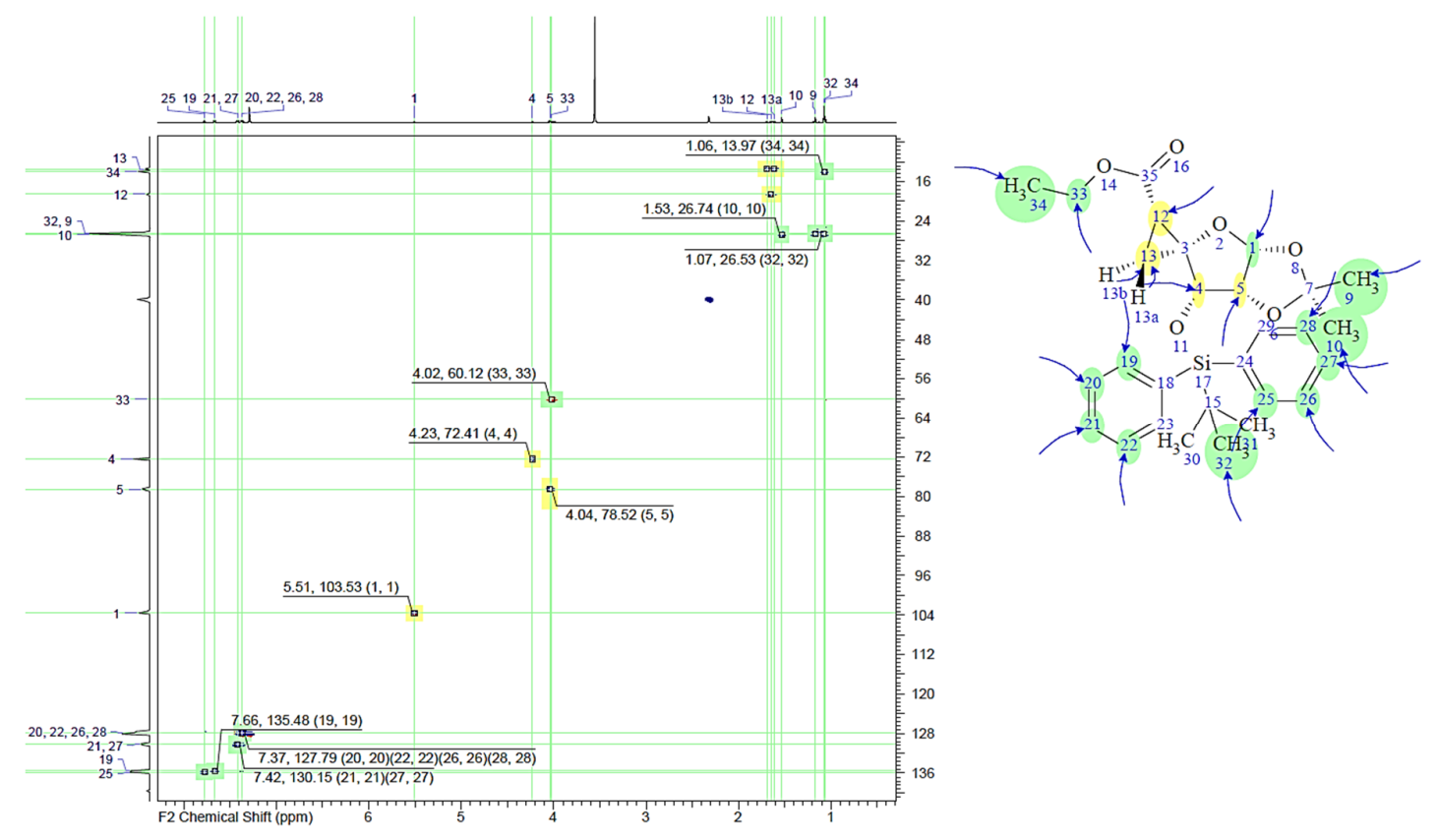

\section{X-ray crystal structure of compound $\mathbf{1 0}$}

For the structure of $\mathbf{1 0}$, X-ray intensity data were collected at $100 \mathrm{~K}$, on a Rigaku Oxford Diffraction Supernova Dual Source ( $\mathrm{Cu}$ at zero) diffractometer equipped with an Atlas CCD detector using $\omega$ scans and CuK $\alpha(\lambda=1.54184 \AA)$ radiation. The images were interpreted and integrated with the program CrysAlisPro. ${ }^{3}$ Using Olex $2,{ }^{4}$ the structure was solved by direct methods using the ShelXT structure solution program and refined by full-matrix least-squares on $F^{2}$ using the ShelXL program package. ${ }^{5,6}$ Non-hydrogen atoms were anisotropically refined and the hydrogen atoms in the riding mode with isotropic temperature factors fixed at 1.2 times $\mathrm{U}(\mathrm{eq})$ of the parent atoms (1.5 times for methyl and hydroxyl groups). For $\mathbf{1 0}$, the absolute configuration was determined with chirality at $\mathrm{C} 6$ $(R), C 7(R), C 8(S), C 9(S)$ and $C 11(R)$, showing a refined Flack parameter of $0.05(5)$.

Crystal data for compound 10. $\mathrm{C}_{13} \mathrm{H}_{19} \mathrm{~N}_{5} \mathrm{O}_{5}, \mathrm{M}=325.33 .45$, monoclinic, space group $\mathrm{P} 2_{1}$ (No. 4), $\mathrm{a}=$ 8.65020(10) $\AA, b=8.25480(10) \AA, c=10.16880(10) \AA, b=94.4770(10)^{\circ}, V=723.895(14) \AA^{3}, Z=2, T=$ $100 \mathrm{~K}, \rho_{\text {calc }}=1.493 \mathrm{~g} \mathrm{~cm}^{-3}, \mu(\mathrm{Cu}-\mathrm{K} \alpha)=0.985 \mathrm{~mm}^{-1}, \mathrm{~F}(000)=344,13732$ reflections measured, 2885 unique $\left(R_{\text {int }}=0.0191\right)$ which were used in all calculations. The final $R 1$ was $0.0263(I>2 \sigma(I))$ and wR2 was 0.0687 (all data).

\footnotetext{
${ }^{3}$ Rigaku Oxford Diffraction, (2019), CrysAlisPro Software system, version 1.171.40.67a, Rigaku Corporation, Oxford, UK.

${ }^{4}$ Dolomanov, O.V.; Bourhis, L.J.; Gildea, R.J.; Howard, J.A.K.; Puschmann, H. J. Appl. Crystallogr. 2009, 42, 339-341.

${ }^{5}$ Sheldrick, G.M. Acta Crystallogr. 2015, A71, 3-8.

${ }^{6}$ Sheldrick, G.M. Acta Crystallogr. 2015, C71, 3-8.
} 
CCDC 2094293 contains the supplementary crystallographic data for this paper. These data can be obtained free of charge from The Cambridge Crystallographic Data Centre via https://www.ccdc.cam.ac.uk/structures/.

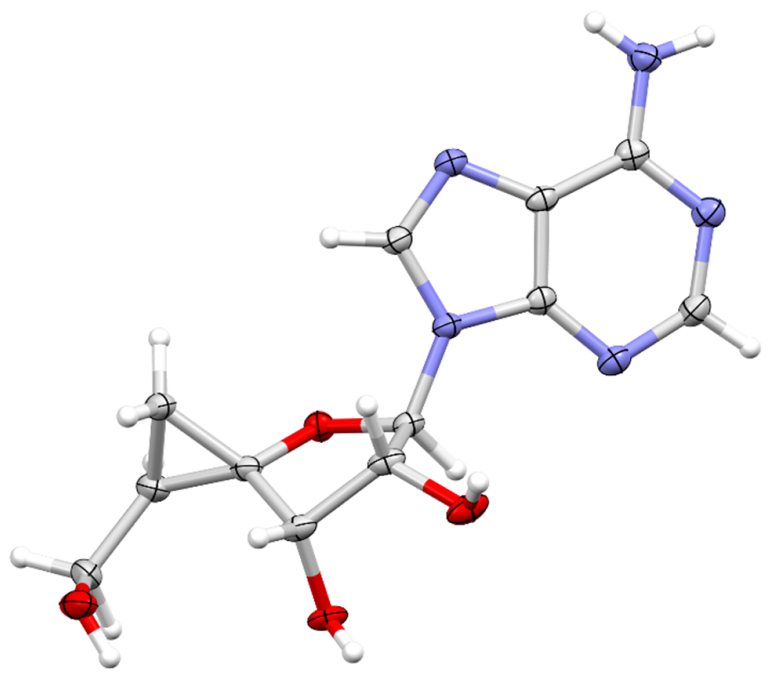

Figure S1. M olecular structure of $\mathbf{1 0}$, showing thermal displacement ellipsoids at the $50 \%$ probability level. Disorder of the methoxy group and a methanol solvent molecule are omitted for clarity. 